\title{
Equation de Navier-Stokes avec densité et viscosité variables dans l'espace critique
}

Hammadi Abidi

\begin{abstract}
In this article, we show that the Navier-Stokes system with variable density and viscosity is locally well-posed in the Besov space

$$
\dot{B}_{p 1}^{\frac{N}{p}}\left(\mathbb{R}^{N}\right) \times\left(\dot{B}_{p 1}^{\frac{N}{p}-1}\left(\mathbb{R}^{N}\right)\right)^{N},
$$

for $1<p \leq N$ when the initial density approaches a strictly positive constant. This result generalizes the work by R. Danchin for the case where the viscosity is constant and $p=2$ (see [8]). Moreover, we prove existence and uniqueness in the Sobolev space

$$
H^{\frac{N}{2}+\alpha}\left(\mathbb{R}^{N}\right) \times\left(H^{\frac{N}{2}-1+\alpha}\left(\mathbb{R}^{N}\right)\right)^{N}
$$

for $\alpha>0$, generalizing R. Danchin's result for the case where viscosity is constant (see [7]).
\end{abstract}

\section{Introduction}

La description mathématique de l'état d'un fluide en mouvement se fait au moyen de fonctions déterminant la distribution de la vitesse du fluide $u$ et de quelques grandeurs thermodynamiques, par exemple la pression $\Pi$ et la densité $\rho$. Il convient de noter qu'en général il y a deux types d'écoulements de fluides qui se présentent. Le fluide parfait suppose une évolution libre des particules, c'est-à-dire qu'elles n'entrent pas en collision dans leurs parcours. Par contre dans le fluide visqueux on tient compte d'éventuelles interactions

2000 Mathematics Subject Classification : Primary: 35Q30; Secondary: 35B30, 76D03, $76 \mathrm{D} 05$.

Keywords : Inhomogenous fluid, existence, uniqueness. 
qui ont tendance à dissiper l'énergie et, par conséquent, à stabiliser le fluide. Ceci est modélisé par un terme appelé viscosité. Lorsque le fluide est inhomogène et incompressible, son écoulement est donné par l'équation suivante :

$$
\rho\left(\partial_{t} u+u \cdot \nabla u\right)-\operatorname{div}(\mu \mathcal{M})+\nabla \Pi=0, \quad \operatorname{div} u=0
$$

avec $\mathcal{M}=\nabla u+{ }^{t} \nabla u$ et $\mu$ le coefficient de viscosité qui est strictement positif et dépend généralement de la pression et de la température. Dans beaucoup de situations physiques nous avons affaire à un fluide qui n'est pas homogène, c'est-à-dire, que sa composition est faite d'un mélange de substances. Ainsi pour mieux décrire le fluide nous devons connaître la concentration $C$ en chaque point de chacune des substances en jeu. Soit $\rho$ la densité totale du fluide, alors en faisant un bilan de la masse qui entre et qui sort d'un volume microscopique fixe, nous parvenons à démontrer que la densité satisfait l'équation suivante

$$
\partial_{t} \rho+\operatorname{div}(\rho u)=0 .
$$

L'équation du mouvement du fluide reste la même c'est-à-dire

$$
\rho\left(\partial_{t} u+u \cdot \nabla u\right)-\operatorname{div}(\mu \mathcal{M})+\nabla \Pi=0,
$$

mais nous devons tenir compte de la dépendance de $\mu$ par rapport à la concentration des particules (pour plus de détails voir par exemple [16]). Donc on conclut qu'un fluide non homogène est donné par un champ de vecteurs $u$ dépendant du temps qui est supposé décrire la vitesse d'une particule située au point $x \in \mathbb{R}^{N}$ à l'instant $t \in \mathbb{R}_{+}$et sa densité $\rho=$ $\rho(t, x) \geq 0$. Le système est le suivant

$$
\left\{\begin{array}{l}
\partial_{t} \rho+\operatorname{div}(\rho u)=0 \\
\partial_{t}(\rho u)+\operatorname{div}(\rho u \otimes u)-\operatorname{div}(\mu(\rho) \mathcal{M})+\nabla \Pi=\rho f \\
\operatorname{div} u=0 \\
(\rho, u)_{\mid t=0}=\left(\rho_{0}, u_{0}\right),
\end{array}\right.
$$

où $\mu$ est une fonction positive désignant la viscosité du fluide vérifie

$$
0<\underline{\mu} \leq \mu, \quad \text { et } \quad \mu \in C^{\infty} .
$$

$\Pi(t, x)$, représente la pression, $\operatorname{div}(\rho u \otimes u)^{j}=\sum_{k} \partial_{k}\left(\rho u^{j} u^{k}\right)$ et $f$ représente la densité de forces extérieures. Notons que la condition de divergence nulle traduit l'incompressibilité du fluide.

Avant d'aborder le vif du sujet, commençons par rappeler quelques résultats consacrés à l'étude de ce système lorsque $\mu$ est une constante strictement positive. Le premier auteur qui a montré l'existence globale de solutions 
faibles est V. Kazhikov [15] (voir aussi [1]). Dans le même esprit J. Simon [23] a démontré que (INS) admet de solutions faibles globales à la Leray dans des espaces de type $L^{2}$, dans un ouvert borné de $\mathbb{R}^{3}$ avec condition de Dirichlet. Un peu plus tard E. Fernández-Cara et F. Guillén [13] ont démontré un résultat semblable sur un ouvert non borné. Tous ces résultats reposent sur la relation suivante (valable pour les solutions régulieres)

$$
\begin{aligned}
\|\sqrt{\rho} u(t)\|_{L^{2}}^{2}+2 \mu \int_{0}^{t} & \|\nabla u(\tau)\|_{L^{2}}^{2} d \tau \\
& =\left\|\sqrt{\rho_{0}} u_{0}\right\|_{L^{2}}^{2}+2 \int_{0}^{t}(\rho f \cdot u)(\tau, x) d x d \tau,
\end{aligned}
$$

et sur des méthodes de compacité. Remarquons que (1.2) ressemble fort à l'inégalité d'énergie de $\left(\mathrm{NS}_{\mu}\right)$

$$
\left(\mathrm{NS}_{\mu}\right) \quad\left\{\begin{array}{l}
\partial_{t} u+u \cdot \nabla u-\mu \Delta u=-\nabla \Pi \\
\operatorname{div} u=0 \\
u_{\mid t=0}=u_{0} .
\end{array}\right.
$$

Lorsque $\mu$ dépend de la densité, on a également existence de solutions faibles "à la Leray" [17] : là encore, la preuve repose sur des méthodes de compacité basées sur l'inégalité d'énergie suivante

$$
\begin{aligned}
\int_{\mathbb{R}^{N}} \rho\left|u-u_{\infty}\right|^{2} d x & +\int_{0}^{t} \int_{\mathbb{R}^{N}} \mu\left(\partial_{i} u_{j}+\partial_{j} u_{i}\right)^{2} d x d s \\
& \leq \int_{\mathbb{R}^{N}} \rho_{0}\left|u_{0}-u_{\infty}\right|^{2} d x+2 \int_{0}^{t} \int_{\mathbb{R}^{N}} \rho f\left(u-u_{\infty}\right) d x d s
\end{aligned}
$$

avec $u \longrightarrow u_{\infty}$ lorsque $|x| \longrightarrow+\infty$, pour tout $t \geq 0$ (voir [12] et [18] pour plus de détails).

Les résultats que nous avons cités sont consacrés à l'existence de solutions faibles. Mais récemment $\mathrm{R}$. Danchin a généralisé certains résultats connus pour le système de Navier-Stokes incompressible homogène $\left(\mathrm{NS}_{\mu}\right)$, lorsque $\mu$ constante comme celui de H. Fujita et T. Kato [14] qui dit que $\left(\mathrm{NS}_{\mu}\right)$ est localement bien posé dans $\left(\dot{H}^{\frac{N}{2}-1}\left(\mathbb{R}^{N}\right)\right)^{N}$. Plus précisement, il montre que (INS) est localement bien posé pour

$$
\left(\rho_{0}-c t e, u_{0}\right) \in H^{\frac{N}{2}+\alpha} \times\left(H^{\frac{N}{2}+\alpha-1}\right)^{N}
$$

avec $\alpha>0$ (voir [7]). Il prouve également que ce système est bien posé dans l'espace critique $\dot{B}_{21}^{\frac{N}{2}} \times\left(\dot{B}_{21}^{\frac{N}{2}-1}\right)^{N}$ si l'on impose à $\rho_{0}$ une condition de petitesse (c'est-à-dire que la densité initiale est proche d'une constante 
strictement positive) [8]. Lorsqu'on est en dimension deux d'espace, le même auteur démontre un résultat analogue à celui de Leray [17] qui dit que $\left(\mathrm{NS}_{\mu}\right)$ est globalement bien posé dans $L^{2}$. Plus exactement il établit dans [7] que (INS) est globalement bien posé dans $H^{1+\alpha} \times\left(H^{\alpha}\right)^{2}$, avec $\alpha>0$.

La question à laquelle nous allons tenter de répondre dans ce travail est la suivante : peut-on généraliser le résultat cité de [8] lorsque la viscosité $\mu$ n'est plus constante mais dépend de la densité et vérifie (1.1), dans les espaces de Besov construits sur les espaces de Lebesgue $L^{p}$ ?

La motivation physique de cette question est le fait que la viscosité dépend en général de la densité. En ce qui concerne l'aspect mathématique on cherche à abaisser l'exigence de régularité sur les données initiales.

En ce qui concerne les solutions fortes qui feront l'objet de cet article, nous réussissons à prouver que (INS) est localement bien posé dans des espaces de Besov construits sur les espaces de Lebesgue $L^{p}$. La preuve est intrinsèquement liée à deux faits principaux. Le premier un lemme d'analyse harmonique dû à $R$. Danchin et par lequel, on généralise l'inégalité de Poincaré (voir [10] et [21]). Tandis que le second est un lemme de stabilité de l'espace $B_{p r}^{s}$ pour $s>0$ par l'action d'une fonction $C^{\infty}$ à support compact (voir par exemple le livre de Y. Meyer [19]).

Dans la suite on supposera que la densité du fluide est uniforme et non nulle au voisinage de l'infini, ce qui veut dire qu'elle tend vers une valeur finie à l'infini que l'on peut prendre égale à 1 .

Pour étudier le système (INS) nous effectuons le changement d'inconnue $a=\frac{1}{\rho}-1$, qui est valable si $\rho(t, x)>0$, mais cette hypothèse est vraie dès que $\inf _{x} \rho(0, x)>0$ car nous avons par le principe du maximum $\inf _{x} \rho(t, x)=$ $\inf _{x} \rho(0, x)$. Le système (INS) devient

$$
\widetilde{(\mathrm{INS})}\left\{\begin{array}{l}
\partial_{t} a+u \cdot \nabla a=0 \\
\partial_{t} u+u \cdot \nabla u+(1+a)\{\nabla \Pi-\operatorname{div}(\widetilde{\mu}(a) \mathcal{M})\}=f \\
\operatorname{div} u=0 \\
(a, u)_{\mid t=0}=\left(a_{0}, u_{0}\right)
\end{array}\right.
$$

avec $\widetilde{\mu}(a)=\mu\left(\frac{1}{1+a}\right)$.

Avant d'énoncer les résultats, on rappelle que l'opérateur de Leray $\mathcal{P}$ est le projecteur orthogonal sur les champs à divergence nulle. On note $\mathcal{Q}=I-\mathcal{P}$ le projecteur sur les champs de type gradient.

Nos résultats principaux sont les suivants (concernant la définition de l'espace de Besov voir la définition 2.1) : 
Théorème 1.1. Soient $1<p<2 N$, $\mu$ vérifie (1.1), $u_{0} \in\left(\dot{B}_{p 1}^{\frac{N}{p}-1}\left(\mathbb{R}^{N}\right)\right)^{N}$ avec $\operatorname{div} u=0, f \in\left(L_{\text {loc }}^{1}\left(\mathbb{R}_{+} ; \dot{B}_{p 1}^{\frac{N}{p}-1}\left(\mathbb{R}^{N}\right)\right)\right)^{N}$ tel que $\mathcal{Q} f$ appartenant $\grave{a}$ $\left(L_{\text {loc }}^{2}\left(\mathbb{R}_{+} ; \dot{B}_{p 1}^{\frac{N}{p}-2}\left(\mathbb{R}^{N}\right)\right)\right)^{N}$ et $a_{0} \in \dot{B}_{p 1}^{\frac{N}{p}}\left(\mathbb{R}^{N}\right)$. Alors il existe une constante positive $c$ dépendant de $N, p$ et de la fonction $\mu$ telle que si

$$
\left\|a_{0}\right\|_{\dot{B}_{p 1}^{\frac{N}{p}}} \leq c
$$

il existe un $T \in(0,+\infty]$ tel que le système ( $\widetilde{\mathrm{INS}})$ admette une solution $(a, u, \nabla \Pi)$ vérifiant

$$
\begin{gathered}
a \in C_{b}\left([0, T) ; \dot{B}_{p 1}^{\frac{N}{p}}\right), u \in\left(C_{b}\left([0, T) ; \dot{B}_{p 1}^{\frac{N}{p}-1}\right) \cap L_{T}^{1}\left(\dot{B}_{p 1}^{\frac{N}{p}+1}\right)\right)^{N} \\
\text { et } \nabla \Pi \in\left(L_{T}^{1}\left(\dot{B}_{p 1}^{\frac{N}{p}-1}\right)\right)^{N} .
\end{gathered}
$$

De plus cette solution est unique lorsque $1<p \leq N$. D'autre part, il existe une constante $c^{\prime}$ strictement positive dépendant de $N, \mu$ et $p$ telle que si

$$
\left\|u_{0}\right\|_{\dot{B}_{p 1}^{\frac{N}{p}-1}}+\|f\|_{L^{1}\left(\mathbb{R}_{+} ; \dot{B}_{p 1}^{\frac{N}{p}-1}\right)} \leq c^{\prime} \mu^{1}
$$

alors $T=+\infty$.

En fait on peut se débarrasser de l'hypothèse de petitesse de la donnée initiale $a_{0}$ mais le prix qu'on paye touche la classe des données initiales que l'on doit prendre plus régulières. Ceci est précisé dans le théorème suivant.

Théorème 1.2. Soit $\alpha$ un réel strictement positif et $\mu$ vérifie (1.1). On suppose que $a_{0} \in H^{\frac{N}{2}+\alpha}\left(\mathbb{R}^{N}\right)$ vérifie $\underline{a} \stackrel{\text { déf }}{=} \inf _{x}\left(1+a_{0}\right)>0$ (et de plus $\nabla a_{0} \in$ $\left(L^{\infty}\left(\mathbb{R}^{N}\right)\right)^{N}$ si $\left.\alpha=1\right)$. Soit $u_{0}$ un champ de vecteurs à divergence nulle avec coefficients dans $H^{\frac{N}{2}-1+\alpha}\left(\mathbb{R}^{N}\right)$ et $f \in\left(\widetilde{L}_{\text {loc }}^{1}\left(\mathbb{R}_{+} ; H^{\frac{N}{2}-1+\alpha}\left(\mathbb{R}^{N}\right)\right)\right)^{N}$. Alors il existe $T$ strictement positif tel que le système $(\widetilde{\mathrm{INS}})$ admette une unique solution sur $[0, T]$. Plus précisement, on a

$$
\begin{gathered}
a \in \widetilde{C}_{T}\left(H^{\frac{N}{2}+\alpha}\right), u \in\left(\widetilde{L}_{T}^{1}\left(H^{\frac{N}{2}+1+\alpha}\right) \cap \widetilde{C}_{T}\left(H^{\frac{N}{2}-1+\alpha}\right)\right)^{N} \\
\text { et } \nabla \Pi \in\left(\widetilde{L}_{T}^{1}\left(H^{\frac{N}{2}-1+\alpha}\right)\right)^{N} .
\end{gathered}
$$

Nous allons maintenant donner le plan de l'article :

- Premièrement nous rappelons les notions des bases de la théorie de Littlewood-Paley.

- La troisième section est consacrée à l'unicité dans l'espace de Besov.

- La quatrième section est dédiée à l'existence de solutions. 


\section{Notations et définitions}

\subsection{Notations}

On dit que $A \lesssim B$ s'il existe une contante $c$ strictement positive telle que $A \leq c B$, et $A \approx B$ si $A \lesssim B$ et $B \lesssim A$.

Soient $X$ un espace de Banach et $p \in[1, \infty]$, on désigne par $L^{p}(0, T ; X)$ l'ensemble des fonctions $f$ mesurables sur $(0, T)$ à valeurs dans $X$, telles que $t \longmapsto\|f(t)\|_{X}$ appartient à $L^{p}(0, T)$. On note $C([0, T) ; X)$ l'espace des fonctions continues de $[0, T)$ à valeurs dans $X, C_{b}([0, T) ; X) \stackrel{\text { déf }}{=} C([0, T) ; X) \cap$ $L^{\infty}(0, T ; X)$ et $\widetilde{C}_{T}(X) \stackrel{\text { déf }}{=} C([0, T) ; X) \cap \widetilde{L}^{\infty}(0, T ; X)$.

Enfin soit $\mu^{1}=\mu(1), \widetilde{\mu}(a)=\mu\left(\frac{1}{1+a}\right), a \vee b=\inf (a, b)$ et pour $1 \leq p \leq \infty$, on désigne par $p^{\prime}$ l'exposant conjugué de $p$ défini par $\frac{1}{p}+\frac{1}{p^{\prime}}=1$.

\subsection{Théorie de Littlewood-Paley}

Dans cette section, nous allons rappeler brièvement la théorie de LittlewoodPaley et définir les espaces fonctionnels dans lesquels nous allons travailler. Pour cela, nous utilisons une décomposition dyadique de l'unité (voir par exemple [4]). Soit $C \subset \mathbb{R}^{N}$, la couronne de centre 0 de petit rayon $\frac{3}{4}$, de grand rayon $\frac{8}{3}$. Il existe alors deux fonctions positives radiales $\chi$ et $\varphi$ appartenant respectivement à $C_{0}^{\infty}\left(B\left(0, \frac{4}{3}\right)\right)$ et à $C_{0}^{\infty}(C)$ telles que :

$$
\sum_{q \in \mathbb{Z}} \varphi\left(2^{-q} \xi\right)=1 \quad \forall \xi \neq 0 \quad \text { et } \quad \chi(\xi)+\sum_{q \in \mathbb{N}} \varphi\left(2^{-q} \xi\right)=1 \quad \forall \xi \in \mathbb{R}^{N} .
$$

On définit les opérateurs $\Delta_{q}, S_{q}, \dot{S}_{q}$ et $\dot{\Delta}_{q}$ de $\mathcal{L}\left(\mathcal{S}^{\prime} ; C^{\infty}\right)$ par :

$$
\begin{aligned}
\Delta_{q} u & =0 \quad \text { si } \quad q \leq-2, \quad \Delta_{-1} u=\chi(D) u=\tilde{h} * u, \\
\Delta_{q} u & =\varphi\left(2^{-q} D\right) u=\dot{\Delta}_{q} u=2^{N q} h\left(2^{q} \cdot\right) * u \quad \text { si } \quad q \geq 0, \\
\dot{\Delta}_{q} u & =\varphi\left(2^{-q} D\right) u=2^{N q} h\left(2^{q} \cdot\right) * u \quad \text { pour } \quad q \in \mathbb{Z}, \\
\dot{S}_{q} u & =\chi\left(2^{-q} D\right) u=\sum_{p \leq q-1} \dot{\Delta}_{p} u=2^{N q} \tilde{h}\left(2^{q} \cdot\right) * u \\
\text { (resp. } \mathrm{S}_{\mathrm{q}} \mathrm{u} & \left.=\chi\left(2^{-q} D\right) u=\sum_{p \leq q-1} \Delta_{p} u=2^{N q} \tilde{h}\left(2^{q} \cdot\right) * u\right)
\end{aligned}
$$

avec $: h=\mathcal{F}^{-1} \varphi$ et $\tilde{h}=\mathcal{F}^{-1} \chi$. De plus, on a :

$$
u=\sum_{q \in \mathbb{Z}} \dot{\Delta}_{q} u \quad \forall u \in \mathcal{S}^{\prime}\left(\mathbb{R}^{N}\right) / \mathcal{P}\left[\mathbb{R}^{N}\right] \quad \text { et } \quad u=\sum_{q \in \mathbb{Z}} \Delta_{q} u \quad \forall u \in \mathcal{S}^{\prime}\left(\mathbb{R}^{N}\right)
$$

où $\mathcal{P}\left[\mathbb{R}^{N}\right]$ est l'ensemble des polynômes (voir par exemple [20]). 
De plus, la décomposition de Littlewood-Paley vérifie la propriété de presque orthogonalité :

$$
\begin{aligned}
& \dot{\Delta}_{k} \dot{\Delta}_{q} u \equiv 0 \text { si }|k-q| \geq 2 \text { et } \dot{\Delta}_{k}\left(\dot{S}_{q-1} u \dot{\Delta}_{q} u\right) \equiv 0 \text { si }|k-q| \geq 5 \\
& \left(\text { resp. } \Delta_{k} \Delta_{q} u \equiv 0 \text { si }|k-q| \geq 2 \text { et } \Delta_{k}\left(S_{q-1} u \Delta_{q} u\right) \equiv 0 \text { si }|k-q| \geq 5\right) .
\end{aligned}
$$

Définition 2.1. Soient $s \in \mathbb{R},(p, r) \in[1,+\infty]^{2}$ et $u \in \mathcal{S}^{\prime}\left(\mathbb{R}^{N}\right)$, on note

$$
\|u\|_{\dot{B}_{p r}^{s}} \stackrel{\text { déf }}{=}\left(\sum_{q \in \mathbb{Z}} 2^{r q s}\left\|\dot{\Delta}_{q} u\right\|_{L^{p}}^{r}\right)^{\frac{1}{r}} \quad\left(\text { resp. }\|u\|_{B_{p r}^{s}} \stackrel{\text { déf }}{=}\left(\sum_{q \in \mathbb{Z}} 2^{r q s}\left\|\Delta_{q} u\right\|_{L^{p}}^{r}\right)^{\frac{1}{r}}\right)
$$

avec le changement habituel pour $r=+\infty$, alors pour $s<\frac{N}{p}, r>1$ et $s \leq \frac{N}{p}, r=1$

$$
\dot{B}_{p r}^{s} \stackrel{\text { déf }}{=}\left\{u \in \mathcal{S}^{\prime}\left(\mathbb{R}^{N}\right) \mid\|u\|_{\dot{B}_{p r}^{s}}<\infty\right\},
$$

sinon on définit $\dot{B}_{p r}^{s}$ comme étant l'adhérence dans $\mathcal{S}^{\prime}$ des fonctions appartenant à l'espace de Schwartz pour la norme $\|\cdot\|_{\dot{B}_{p r}^{s}}$

$$
\left(\text { resp. } \quad B_{p r}^{s} \stackrel{\text { déf }}{=}\left\{u \in \mathcal{S}^{\prime}\left(\mathbb{R}^{N}\right) \mid\|u\|_{B_{p r}^{s}}<\infty\right\}\right) .
$$

Pour $p=r=2$ l'espace de Besov non homogène $B_{p r}^{s}$ coïncide avec l'espace de Sobolev $H^{s}$ (voir par exemple [4]).

Comme conséquence de l'inégalité de Bernstein (voir par exemple [4]) et par définition de $\dot{B}_{p r}^{s}$, (resp. $B_{p r}^{s}$ ) on a la proposition suivante

Proposition 2.1. (i) Il existe une constante c strictement positive telle que

$$
c^{-1}\|u\|_{\dot{B}_{p r}^{s}} \leq\|\nabla u\|_{\dot{B}_{p r}^{s-1}} \leq c\|u\|_{\dot{B}_{p r}^{s}} \quad\left(\operatorname{resp} . \quad\|\nabla u\|_{B_{p r}^{s-1}} \lesssim\|u\|_{B_{p r}^{s}}\right) .
$$

(ii) Pour $p_{1} \leq p_{2}$ et $r_{1} \leq r_{2}$, on a

$$
\dot{B}_{p_{1} r_{1}}^{s} \hookrightarrow \dot{B}_{p_{2} r_{2}}^{s-N\left(\frac{1}{p_{1}}-\frac{1}{p_{2}}\right)} \quad\left(\text { resp. } \quad B_{p_{1} r_{1}}^{s} \hookrightarrow B_{p_{2} r_{2}}^{s-N\left(\frac{1}{p_{1}}-\frac{1}{p_{2}}\right)}\right) .
$$

(iii) Si $p \in[1, \infty]$, alors

$$
\dot{B}_{p 1}^{\frac{N}{p}} \hookrightarrow \dot{B}_{p \infty}^{\frac{N}{p}} \cap L^{\infty} \quad\left(\text { resp. } \quad B_{p 1}^{s} \hookrightarrow B_{p \infty}^{\frac{N}{p}} \cap L^{\infty}\right) .
$$

(iv) Interpolation réelle :

$$
\left(\dot{B}_{p r}^{s_{1}}, \dot{B}_{p r}^{s_{2}}\right)_{\theta, r^{\prime}}=\dot{B}_{p r^{\prime}}^{\theta s_{2}+(1-\theta) s_{1}} \quad\left(\operatorname{resp} . \quad\left(B_{p r}^{s_{1}}, B_{p r}^{s_{2}}\right)_{\theta, r^{\prime}}=B_{p r^{\prime}}^{\theta s_{2}+(1-\theta) s_{1}}\right)
$$

pour $0<\theta<1$ (voir par exemple [22]). 
Proposition 2.2. Pour $(r, p) \in[1, \infty]^{2}$ et $s \in \mathbb{R}$, alors on a les inégalités suivantes

$$
\|u v\|_{\dot{B}_{p r}^{s}} \lesssim\|u\|_{L^{\infty}}\|v\|_{\dot{B}_{p r}^{s}}+\|v\|_{L^{\infty}}\|u\|_{\dot{B}_{p r}^{s}} \quad \text { si } \quad s>0 .
$$

Si $s_{1}, s_{2}<\frac{N}{p}$ (resp. $\left.s_{1}, s_{2} \leq \frac{N}{p}\right), s_{1}+s_{2}+N \min \left(0,1-\frac{2}{p}\right)>0$, alors

$$
\begin{gathered}
\|u v\|_{\dot{B}_{p r}^{s_{1}+s_{2}-\frac{N}{p}}} \lesssim\|u\|_{\dot{B}_{p r}^{s_{1}}}\|v\|_{\dot{B}_{p \infty}^{s_{2}}} \\
\left(\text { resp. pour } r=1 \quad\|u v\|_{\dot{B}_{p 1}^{s_{1}+s_{2}-\frac{N}{p}}} \lesssim\|u\|_{\dot{B}_{p 1}^{s_{1}}}\|v\|_{\dot{B}_{p 1}^{s_{2}}}\right),
\end{gathered}
$$

si $|s|<\frac{N}{p}$ pour $p \geq 2$ et $-\frac{N}{p^{\prime}}<s<\frac{N}{p}$ sinon, alors

$$
\|u v\|_{\dot{B}_{p r}^{s}} \lesssim\|u\|_{\dot{B}_{p r}^{s}}\|v\|_{\dot{B}_{p \infty}^{N} \cap L^{\infty}} .
$$

L'inégalité (2.4) reste vrai dans le cas limite (c'est-à-dire si $s_{1}+s_{2}=0$ ) pour $2 \leq p$ (voir par exemple [22]). Plus précisément on a

$$
\|u v\|_{\dot{B}_{p}^{-\frac{N}{p}}} \lesssim\|u\|_{\dot{B}_{p 1}^{s}}\|v\|_{\dot{B}_{p \infty}^{-s}} \quad \text { si } \quad s \in\left(-\frac{N}{p}, \frac{N}{p}\right],
$$

et

$$
\|u v\|_{\dot{B}_{p \infty}^{-\frac{N}{p}}} \lesssim\|u\|_{\dot{B}_{p \infty}^{\frac{N}{p} \cap L^{\infty}}}\|v\|_{\dot{B}_{p 1}^{-\frac{N}{p}}} \text { si } \quad 2 \leq p .
$$

(2.8) $\|u v\|_{\dot{B}_{p r}^{s}} \lesssim\|u\|_{L^{2} \cap \dot{B}_{2 r}^{s+N-\frac{N}{p}}}\|v\|_{L^{2} \cap \dot{B}_{2 r}^{s+N-\frac{N}{p}}} \quad$ dès que $s+N-\frac{N}{p}>0$.

Preuve. Pour montrer (2.8), on utilise la décomposition de J.-M. Bony (voir [3]), on a

$$
\begin{gathered}
u v=T_{u} v+T_{v} u+R(u, v), \quad \text { avec } \quad T_{u} v \stackrel{\text { déf }}{=} \sum_{q \in \mathbb{Z}} \dot{S}_{q-1} u \dot{\Delta}_{q} v \\
R(u, v) \stackrel{\text { déf }}{=} \sum_{q \in \mathbb{Z}} \dot{\Delta}_{q} u\left(\dot{\Delta}_{q-1}+\dot{\Delta}_{q}+\dot{\Delta}_{q+1}\right) v \quad \text { et } \quad T_{u}^{\prime} v \stackrel{\text { déf }}{=} T_{u} v+R(u, v) .
\end{gathered}
$$

Grâce à (2.1), on a

$$
\dot{\Delta}_{k}\left(T_{u} v\right)=\sum_{|k-q| \leq 4} \dot{\Delta}_{k}\left(\dot{S}_{q-1} u \dot{\Delta}_{q} v\right)
$$

alors les inégalités de Bernstein et de Hölder, impliquent

$$
\begin{aligned}
& \left\|\dot{\Delta}_{k}\left(T_{u} v\right)\right\|_{L^{p}} \lesssim 2^{k N\left(1-\frac{1}{p}\right)}\left\|\dot{\Delta}_{k}\left(T_{u} v\right)\right\|_{L^{1}} \\
& \quad \lesssim 2^{k N\left(1-\frac{1}{p}\right)} \sum_{|k-q| \leq 4}\left\|\dot{S}_{q-1} u\right\|_{L^{2}}\left\|\dot{\Delta}_{q} v\right\|_{L^{2}} \lesssim\|u\|_{L^{2}} 2^{k N\left(1-\frac{1}{p}\right)} \sum_{|k-q| \leq 4}\left\|\dot{\Delta}_{q} v\right\|_{L^{2}} .
\end{aligned}
$$


D'où à partir de l'inégalité de Young on en déduit la majoration suivante

$$
\left\|T_{u} v\right\|_{\dot{B}_{p r}^{s}} \lesssim\|u\|_{L^{2}}\|v\|_{\dot{B}_{2 r}^{s+N-\frac{N}{p}}} .
$$

De même, on a

$$
\left\|T_{v} u\right\|_{\dot{B}_{p r}^{s}} \lesssim\|v\|_{L^{2}}\|u\|_{\dot{B}_{2 r}^{s+N-\frac{N}{p}}} .
$$

Par définition de l'opérateur $\dot{\Delta}_{\lambda}$, on a

$$
\dot{\Delta}_{\lambda} R(u, v)=\sum_{\lambda-k \leq 3} \sum_{|k-q| \leq 1} \dot{\Delta}_{\lambda}\left(\dot{\Delta}_{k} u \dot{\Delta}_{q} v\right) .
$$

Les inégalités de Bernstein et de Hölder impliquent

$$
\left\|\dot{\Delta}_{\lambda} R(u, v)\right\|_{L^{p}} \lesssim 2^{\lambda N\left(1-\frac{1}{p}\right)} \sum_{\lambda-k \leq 3} \sum_{|k-q| \leq 1}\left\|\dot{\Delta}_{k} u\right\|_{L^{2}}\left\|\dot{\Delta}_{q} v\right\|_{L^{2}} .
$$

Ainsi l'inégalité de Young conduit à la majoration suivante

$$
\|R(u, v)\|_{\dot{B}_{p r}^{s}} \lesssim\|u\|_{L^{2}}\|v\|_{\dot{B}_{2 r}^{s+N-\frac{N}{p}}} \quad \text { dès que } \quad s+N-\frac{N}{p}>0 .
$$

D'où le résultat.

Pour les espaces de Sobolev non homogènes, on a aussi une proposition qui est équivalente à la proposition 2.2 (voir par exemple [9, proposition 1.3])

\section{Proposition 2.3.}

$$
\begin{aligned}
& \|u v\|_{H^{s}} \lesssim\|u\|_{L^{\infty}}\|v\|_{H^{s}}+\|v\|_{L^{\infty}}\|u\|_{H^{s}} \quad \text { si } \quad s \geq 0, \\
& \|u v\|_{H^{s_{1}}} \lesssim\|u\|_{H^{s_{1}}}\|v\|_{H^{s_{2}}} \quad \text { si } \quad s_{1}+s_{2}>0, \quad s_{1} \leq \frac{N}{2} \quad \text { et } \quad s_{2}>\frac{N}{2} \text {, } \\
& \|u v\|_{H^{s_{1}+s_{2}-\frac{N}{2}}} \lesssim\|u\|_{H^{s_{1}}}\|v\|_{H^{s_{2}}} \quad \text { si } \quad s_{1}+s_{2}>0 \quad \text { et } \quad s_{1}, s_{2}<\frac{N}{2} \\
& \|u v\|_{H^{s}} \lesssim\|u\|_{H^{s}}\|v\|_{H^{\frac{N}{2}} \cap L^{\infty}} \quad \text { si } \quad|s|<\frac{N}{2} .
\end{aligned}
$$

Remarque 2.1. La proposition 2.2 (resp. la proposition 2.3) montre que $\dot{B}_{p r}^{s} \cap L^{\infty}$ est une algèbre pour $s>0$. De même pour $\dot{B}_{p 1}^{\frac{N}{p}}$ si $p<\infty$ (resp. $H^{s}$ si $\left.s>\frac{N}{2}\right)$.

Pour résoudre l'équation ( $\widetilde{\mathrm{INS}})$ nous utiliserons l'effet régularisant de l'équation de la chaleur découvert par J.-Y. Chemin et N. Lerner dans [6]. Pour cela on introduit les espaces $\widetilde{L}_{T}^{\rho}\left(\dot{B}_{p r}^{s}\right)$. 
Définition 2.2. Soient $s \in \mathbb{R},(r, \rho, p) \in[1,+\infty]^{3}$ et $\left.\left.T \in\right] 0,+\infty\right]$, on dit alors que $f \in \widetilde{L}_{T}^{\rho}\left(\dot{B}_{p r}^{s}\right)$, si

$$
\|f\|_{\widetilde{L}_{T}^{\rho}\left(\dot{B}_{p r}^{s}\right)} \stackrel{\text { déf }}{=}\left(\sum_{q \in \mathbb{Z}} 2^{q r s}\left(\int_{0}^{T}\left\|\dot{\Delta}_{q} f(t)\right\|_{L^{p}}^{\rho} d t\right)^{\frac{r}{\rho}}\right)^{\frac{1}{r}}<\infty
$$

$\left[\right.$ resp. $f \in \widetilde{L}_{T}^{\rho}\left(H^{s}\right)$, si $\left.\|f\|_{\widetilde{L}_{T}^{\rho}\left(H^{s}\right)} \stackrel{\text { déf }}{=}\left(\sum_{q \in \mathbb{Z}} 2^{2 q s}\left(\int_{0}^{T}\left\|\Delta_{q} f(t)\right\|_{L^{2}}^{\rho} d t\right)^{\frac{2}{\rho}}\right)^{\frac{1}{2}}<\infty\right]$, avec le changement usuel si $r=\infty$.

Remarque 2.2. Les fonctionnelles $\|\cdot\|_{\widetilde{L}_{T}^{\rho}\left(\dot{B}_{p r}^{s}\right)}$ ne sont des normes que si $s<\frac{N}{p}\left(\right.$ ou $s \leq \frac{N}{p}$ si $\left.r=1\right)$.

Pour $\theta \in[0,1]$, on a

$$
\begin{aligned}
\|u\|_{\widetilde{L}_{T}^{\rho}\left(\dot{B}_{p r}^{s}\right)} & \leq\|u\|_{\widetilde{L}_{T}^{\rho_{1}}\left(\dot{B}_{p r}^{s_{1}}\right)}^{\theta}\|u\|_{\widetilde{L}_{T}^{\rho_{2}}\left(\dot{B}_{p r}^{s_{2}}\right)}^{1-\theta} \\
\text { et }\|u\|_{\widetilde{L}_{T}^{\rho}\left(H^{s}\right)} & \leq\|u\|_{\widetilde{L}_{T}^{\rho_{1}}\left(H^{s_{1}}\right)}^{\theta}\|u\|_{\widetilde{L}_{T}^{\rho_{2}}\left(H^{s_{2}}\right)}^{1-\theta}
\end{aligned}
$$

avec $\frac{1}{\rho}=\frac{\theta}{\rho_{1}}+\frac{1-\theta}{\rho_{2}}$ et $s=\theta s_{1}+(1-\theta) s_{2}$.

Remarquons que l'inégalité de Minkowski, implique que $\|u\|_{\widetilde{L}_{T}^{\rho}\left(\dot{B}_{p r}^{s}\right)} \leq\|u\|_{L_{T}^{\rho}\left(\dot{B}_{p r}^{s}\right)}$ si $\rho \leq r \quad\left(\operatorname{resp} .\|u\|_{\widetilde{L}_{T}^{\rho}\left(H^{s}\right)} \leq\|u\|_{L_{T}^{\rho}\left(H^{s}\right)}\right.$ si $\left.\rho \leq 2\right)$, $\|u\|_{L_{T}^{\rho}\left(\dot{B}_{p r}^{s}\right)} \leq\|u\|_{\widetilde{L}_{T}^{\rho}\left(\dot{B}_{p r}^{s}\right)}$ si $r \leq \rho \quad\left(\operatorname{resp} .\|u\|_{L_{T}^{\rho}\left(H^{s}\right)} \leq\|u\|_{\widetilde{L}_{T}^{\rho}\left(H^{s}\right)}\right.$ si $\left.2 \leq \rho\right)$.

De même on a une loi de produit dans ces espaces analogue à celle dans $\dot{B}_{p r}^{s}$ (voir par exemple [5]).

Proposition 2.4. Soient $s>0, r \in[1, \infty]$ et $\frac{1}{\rho_{2}}+\frac{1}{\rho_{3}}=\frac{1}{\rho_{1}}+\frac{1}{\rho_{4}}$, alors

$$
\|u v\|_{\widetilde{L}_{T}^{\rho}\left(\dot{B}_{p r}^{s}\right)} \lesssim\|u\|_{L_{T}^{\rho_{1}\left(L^{\infty}\right)}}\|v\|_{\widetilde{L}_{T}^{\rho_{4}\left(\dot{B}_{p r}^{s}\right)}}+\|v\|_{L_{T}^{\rho_{2}\left(L^{\infty}\right)}}\|u\|_{\widetilde{L}_{T}^{\rho_{3}\left(\dot{B}_{p r}^{s}\right)}} .
$$

Si $s_{1}, s_{2}<\frac{N}{p}$ (resp. $\left.s_{1}, s_{2} \leq \frac{N}{p}\right), s_{1}+s_{2}+N \min \left(0,1-\frac{2}{p}\right)>0$ et $\frac{1}{\rho_{1}}+\frac{1}{\rho_{2}}=\frac{1}{\rho}$, alors

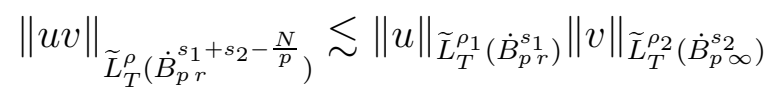

$$
\left(\text { resp. pour } r=1 \quad\|u v\|_{\widetilde{L}_{T}^{\rho}\left(\dot{B}_{p 1}^{\left.s_{1}+s_{2}-\frac{N}{p}\right)}\right.} \lesssim\|u\|_{\widetilde{L}_{T}^{\rho_{1}\left(\dot{B}_{p 1}^{s_{1}}\right)}}\|v\|_{\tilde{L}_{T}^{\rho_{2}\left(\dot{B}_{p 1}^{s_{2}}\right)}}\right) .
$$

Soit $|s|<\frac{N}{p}$ pour $p \geq 2$ et $-\frac{N}{p^{\prime}}<s<\frac{N}{p}$ sinon, alors

$$
\|u v\|_{\tilde{L}_{T}^{\rho}\left(\dot{B}_{p r}^{s}\right)} \lesssim\|u\|_{\widetilde{L}_{T}^{\rho_{1}\left(\dot{B}_{p r}^{s}\right)}}\|v\|_{\widetilde{L}_{T}^{\rho_{2}\left(\dot{B}_{p}^{p}\right) \cap L_{T}^{p}}} \quad \text { avec } \quad \frac{1}{\rho_{1}}+\frac{1}{\rho_{2}}=\frac{1}{\rho} \leq 1 .
$$

Si $2 \leq p, s \in\left(-\frac{N}{p}, \frac{N}{p}\right]$ et $\frac{1}{\rho_{1}}+\frac{1}{\rho_{2}}=\frac{1}{\rho} \leq 1$, on $a$

$$
\|u v\|_{\widetilde{L}_{T}^{\rho}\left(\dot{B}_{p}^{\left.-\frac{N}{p}\right)}\right.} \lesssim\|u\|_{\widetilde{L}_{T}^{\rho_{1}\left(\dot{B}_{p 1}^{s}\right)}}\|v\|_{\widetilde{L}_{T}^{\rho_{2}\left(\dot{B}_{p \infty}^{-s}\right)}} .
$$


Preuve. Pour la démonstration de l'inégalité (2.13) voir [8].

Pour montrer (2.12), on utilise de nouveau la décomposition de J.-M. Bony, on obtient

$$
\left\|\dot{\Delta}_{k}\left(T_{u} v\right)\right\|_{L_{T}^{\rho}\left(L^{p}\right)} \leq \sum_{|k-q| \leq 4}\left\|\dot{\Delta}_{q} v\right\|_{L_{T}^{\rho_{2}}\left(L^{p}\right)}\left\|\dot{S}_{q-1} u\right\|_{L_{T}^{\rho_{1}}\left(L^{\infty}\right)} .
$$

Or l'inégalité de Bernstein implique

$$
\left\|\dot{S}_{q-1} u\right\|_{L_{T}^{\rho_{1}}\left(L^{\infty}\right)} \lesssim \sum_{\lambda \leq q-2} 2^{\lambda\left(\frac{N}{p}-s\right)} 2^{\lambda s}\left\|\dot{\Delta}_{\lambda} u\right\|_{L_{T}^{\rho_{1}}\left(L^{p}\right)}
$$

on injecte cette majoration dans l'inégalité précédente, on trouve

$$
\begin{aligned}
2^{k s}\left\|\dot{\Delta}_{k}\left(T_{u} v\right)\right\|_{L_{T}^{\rho}\left(L^{p}\right)} \lesssim & \sum_{|k-q| \leq 4} 2^{q \frac{N}{p}}\left\|\dot{\Delta}_{q} v\right\|_{L_{T}^{\rho_{2}}\left(L^{p}\right)} \\
& \times \sum_{\lambda \leq q-2} 2^{(q-\lambda)\left(s-\frac{N}{p}\right)} 2^{\lambda s}\left\|\dot{\Delta}_{\lambda} u\right\|_{L_{T}^{\rho_{1}\left(L^{p}\right)}}
\end{aligned}
$$

alors l'inégalité de Young donne l'estimation suivante

$$
\left\|T_{u} v\right\|_{\widetilde{L}_{T}^{\rho}\left(\dot{B}_{p r}^{s}\right)} \lesssim\|u\|_{\widetilde{L}_{T}^{\rho_{1}\left(\dot{B}_{p r}^{s}\right)}}\|v\|_{\widetilde{L}_{T}^{\rho_{2}\left(\dot{B}_{p}^{p}{ }^{p}\right)}} \quad \text { dès que } \quad s<\frac{N}{p} .
$$

Par contre

$$
\left\|T_{v} u\right\|_{\widetilde{L}_{T}^{\rho}\left(\dot{B}_{p r}^{s}\right)} \leq\|u\|_{\tilde{L}_{T}^{\rho_{1}\left(\dot{B}_{p r}^{s}\right)}}\|v\|_{L_{T}^{\rho_{2}\left(L^{\infty}\right)}} \quad \text { pour tout } \quad s \in \mathbb{R} .
$$

Pour estimer le reste, en reprenant les calculs qui ont conduit à majorer le reste de l'inégalité (2.7), on prouve pour $p \geq 2$

$$
\begin{aligned}
2^{\lambda s}\left\|\dot{\Delta}_{\lambda} R(u, v)\right\|_{L_{T}^{\rho}\left(L^{p}\right)} \lesssim & \sum_{\lambda-k \leq 3} 2^{(\lambda-k)\left(s+\frac{N}{p}\right)} 2^{k s}\left\|\dot{\Delta}_{k} u\right\|_{L_{T}^{\rho_{1}}\left(L^{p}\right)} \\
& \times \sum_{|k-q| \leq 1} 2^{q \frac{N}{p}}\left\|\dot{\Delta}_{q} v\right\|_{L_{T}^{\rho_{2}}\left(L^{p}\right)},
\end{aligned}
$$

sinon, c'est-à-dire, $p<2$

$$
\begin{aligned}
2^{\lambda s}\left\|\dot{\Delta}_{\lambda} R(u, v)\right\|_{L_{T}^{\rho}\left(L^{p}\right) \lesssim} & \sum_{\lambda-k \leq 3} 2^{(\lambda-k)\left(s+\frac{N}{p^{\prime}}\right)} 2^{k s}\left\|\dot{\Delta}_{k} u\right\|_{L_{T}^{\rho_{1}\left(L^{p}\right)}} \\
& \times \sum_{|k-q| \leq 1} 2^{q \frac{N}{p}}\left\|\dot{\Delta}_{q} v\right\|_{L_{T}^{\rho_{2}}\left(L^{p}\right)},
\end{aligned}
$$

et par suite lorsque $s>-\frac{N}{p}$ pour $p \geq 2$ et $s>-\frac{N}{p^{\prime}}$ sinon, l'inégalité de Young implique que

$$
\|R(u, v)\|_{\widetilde{L}_{T}^{\rho}\left(\dot{B}_{p r}^{s}\right)} \lesssim\|u\|_{\widetilde{L}_{T}^{\rho_{1}\left(\dot{B}_{p r}^{s}\right)}}\|v\|_{\widetilde{L}_{T}^{\rho_{2}}\left(\dot{B}_{p}^{N}\right)} .
$$


Remarque 2.3. En reprenant les calculs qui ont conduit à (2.7), on prouve que pour $2 \leq p$

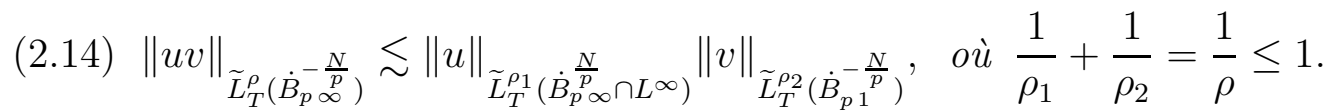

Remarque 2.4. La proposition 2.3 reste vraie dans $\widetilde{L}_{T}^{\rho}\left(H^{s}\right)$ par exemple

$$
\|u v\|_{\widetilde{L}_{T}^{\rho}\left(H^{s_{1}}\right)} \lesssim\|u\|_{\widetilde{L}_{T}^{\rho_{1}}\left(H^{s_{1}}\right)}\|v\|_{\widetilde{L}_{T}^{\rho_{2}}\left(H^{s_{2}}\right)},
$$

si $s_{1}+s_{2}>0, s_{1} \leq \frac{N}{2}$ et $\frac{N}{2}<s_{2}$ avec $\frac{1}{\rho}=\frac{1}{\rho_{1}}+\frac{1}{\rho_{2}}$ (voir [9]).

Lemme 2.1. Soient $m \in[1, \infty], T \in \mathbb{R}_{+}, s \in \mathbb{R}, \alpha>0, \nabla u$ à coefficients dans $\widetilde{L}_{T}^{a}\left(H^{\frac{N}{2}+\alpha-1}\right)$ et $\nabla v$ à coefficients dans $\widetilde{L}_{T}^{b}\left(H^{s-\frac{1}{2} \alpha \vee 1}\right)$ avec $\frac{1}{m}=\frac{1}{a}+\frac{1}{b}$. Alors

$$
\left\|T_{\nabla u} v\right\|_{\widetilde{L}_{T}^{m}\left(H^{s}\right)} \lesssim\|\nabla u\|_{\widetilde{L}_{T}^{a}\left(H^{\frac{N}{2}+\alpha-1}\right)}\|\nabla v\|_{\widetilde{L}_{T}^{b}\left(H^{s-\frac{1}{2} \alpha \vee 1}\right)} .
$$

Preuve. Comme $S_{q} f=\sum_{p \leq q-1} \Delta_{p} f$ et $\Delta_{p} f=0$ si $p \leq-2$, alors

$$
T_{\nabla u} v=\sum_{q \geq 1} S_{q-1} \nabla u \Delta_{q} v
$$

Et par suite l'inégalité (2.1) implique

$$
\Delta_{k}\left(T_{\nabla u} v\right)=\sum_{|k-q| \leq 4} \Delta_{k}\left(S_{q-1} \nabla u \Delta_{q} v\right)
$$

d'où les inégalités de Bernstein et de Hölder donnent (car $q \geq 1$ )

$$
\left\|\Delta_{k}\left(T_{\nabla u} v\right)\right\|_{L_{T}^{m}\left(L^{2}\right)} \lesssim \sum_{|k-q| \leq 4} 2^{-q}\left\|S_{q-1} \nabla u\right\|_{L_{T}^{a}\left(L^{\infty}\right)}\left\|\Delta_{q} \nabla v\right\|_{L^{b}\left(L^{2}\right)},
$$

avec $\frac{1}{m}=\frac{1}{a}+\frac{1}{b}$. Mais de nouveau l'inégalité de Bernstein implique

$$
\left\|S_{q-1} \nabla u\right\|_{L_{T}^{a}\left(L^{\infty}\right)} \lesssim \sum_{-1 \leq \lambda \leq q-2} 2^{\lambda\left(\frac{N}{2}-1+\alpha\right)}\left\|\Delta_{\lambda} \nabla u\right\|_{L_{T}^{a}\left(L^{2}\right)} 2^{\lambda(1-\alpha)},
$$

alors

$$
\left\|S_{q-1} \nabla u\right\|_{L_{T}^{a}\left(L^{\infty}\right)} \lesssim \begin{cases}\|\nabla u\|_{\widetilde{L}_{T}^{a}\left(H^{\frac{N}{2}-1+\alpha}\right)} 2^{q(1-\alpha \vee 1)} & \text { si } \alpha \neq 1 \\ \|\nabla u\|_{\widetilde{L}_{T}^{a}\left(H^{\frac{N}{2}}\right)} \sqrt{q} & \text { si } \alpha=1,\end{cases}
$$

ce qui démontre l'inégalité (2.15).

Il est bien connu que l'espace de Besov $B_{p r}^{s}$ est stable par composition à gauche dès que $s>0$ (voir par exemple [19]). H. Bahouri et J.-Y. Chemin dans [2] ont montré un résultat analogue à celui de [19] dans l'espace $\widetilde{L}_{T}^{\infty}\left(B_{21}^{s}\right)$ basé sur la méthode dite de la première linéarisation de Y. Meyer. Plus exactement, ils obtiennent 
Lemme 2.2. Pour toute fonction $G \in C^{\infty}$ à support compact nulle en 0 et pour tout réel strictement positif $s$, on a

$$
\|G(u)\|_{\widetilde{L}_{T}^{\infty}\left(B_{21}^{s}\right)} \leq C_{u}\|u\|_{\widetilde{L}_{T}^{\infty}\left(B_{21}^{s}\right)}
$$

avec $C_{u} \stackrel{\text { déf }}{=} C\left(1+\|u\|_{L_{T}^{\infty}\left(L^{\infty}\right)}\right)^{[s]+1}$, où $C$ est une constante ne dépendant que de $G$ et de s.

En suivant une démarche strictement analogue à celle de la démontration du lemme 2.2 et en utilisant le fait que l'espace de Besov homogène est défini modulo les polynômes, on obtient le lemme suivant.

Lemme 2.3. Soient $s$ un réel strictement positif, $(p, r, \rho) \in[1, \infty]^{3}, G \in$ $W_{l o c}^{[s]+1, \infty}, T \in(0, \infty]$ et $u \in \widetilde{L}_{T}^{\rho}\left(\dot{B}_{p r}^{s}\right) \cap L_{T}^{\infty}\left(L^{\infty}\right)$, alors

$$
\|G(u)\|_{\widetilde{L}_{T}^{\rho}\left(\dot{B}_{p r}^{s}\right)} \leq C\left(1+\|u\|_{L_{T}^{\infty}\left(L^{\infty}\right)}\right)^{[s]+1}\|u\|_{\widetilde{L}_{T}^{\rho}\left(\dot{B}_{p r}^{s}\right)}
$$

$$
\left(\operatorname{resp} .\|G(u)\|_{\widetilde{L}_{T}^{\rho}\left(B_{p r}^{s}\right)} \leq C\left(1+\|u\|_{L_{T}^{\infty}\left(L^{\infty}\right)}\right)^{[s]+1}\|u\|_{\widetilde{L}_{T}^{\rho}\left(B_{p r}^{s}\right)} \quad \text { si } \quad G(0)=0\right) .
$$

Lemme 2.4. Soient $p, r, \rho$ et $T$ vérifiant les conditions du lemme 2.3 avec $|s|<\frac{N}{p}$ pour $p \geq 2$ sinon $-\frac{N}{p^{\prime}}<s<\frac{N}{p}$ et $G$ appartenant $\grave{a} W_{l o c}^{\left[\frac{N}{p}\right]+2, \infty}$. Alors pour $u$ et $v$ appartenant $\grave{a} L_{T}^{\infty}\left(\dot{B}_{p \infty}^{\frac{N}{p}} \cap L^{\infty}\right)$ tels que $u-v \in \widetilde{L}_{T}^{\rho}\left(\dot{B}_{p r}^{s}\right)$, on a

$$
\begin{aligned}
\|G(u)-G(v)\|_{\widetilde{L}_{T}^{\rho}\left(\dot{B}_{p r}^{s}\right)} \lesssim \| & -v \|_{\widetilde{L}_{T}^{\rho}\left(\dot{B}_{p r}^{s}\right)}\left[\left(1+\|u\|_{L_{T}^{\infty}\left(L^{\infty}\right)}+\|v\|_{L_{T}^{\infty}\left(L^{\infty}\right)}\right){ }^{\left[\frac{N}{p}\right]+1}\right. \\
& \left.\times\left(\|u\|_{L_{T}^{\infty}\left(\dot{B}_{p}^{\frac{N}{p}}\right)}+\|v\|_{L_{T}^{\infty}\left(\dot{B}_{p}^{\frac{N}{p}}\right)}\right)\right] .
\end{aligned}
$$

Preuve. D'après la formule de Taylor avec reste intégral, on a

$$
G(u)-G(v)=\int_{0}^{1}(u-v) G^{\prime}(v+\tau(u-v)) d \tau,
$$

pour conclure il suffit d'utiliser les inégalités (2.12) et (2.17).

Rappelons enfin un résultat d'interpolation logarithmique pour les espaces $\widetilde{L}_{T}^{\rho}\left(\dot{B}_{p r}^{s}\right)$ (voir [8, proposition 1.8]).

Proposition 2.5. Soient $(p, \rho) \in[1, \infty]^{2}, s \in \mathbb{R}$ et $\varepsilon \in(0,1]$, alors

$$
\|u\|_{\widetilde{L}_{T}^{\rho}\left(\dot{B}_{p 1}^{s}\right)} \lesssim \frac{\|u\|_{\widetilde{L}_{T}^{\rho}\left(\dot{B}_{p \infty}^{s}\right)}}{\varepsilon} \log \left(e+\frac{\|u\|_{\tilde{L}_{T}^{\rho}\left(\dot{B}_{p \infty}^{s-\varepsilon}\right)}+\|u\|_{\widetilde{L}_{T}^{\rho}\left(\dot{B}_{p \infty}^{s+\varepsilon}\right)}}{\|u\|_{\widetilde{L}_{T}^{\rho}\left(\dot{B}_{p \infty}^{s}\right)}}\right) .
$$




\section{Unicité}

Dans cette section, nous allons démontrer l'unicité des solutions des équations de Navier-Stokes non homogène dans l'espace de Besov homogène

$$
L_{T}^{\infty}\left(\dot{B}_{p \infty}^{\frac{N}{p}}\right) \times\left(L_{T}^{\infty}\left(\dot{B}_{p 1}^{\frac{N}{p}-1}\right)\right)^{N}
$$

pour $1 \leq p \leq N$. La preuve repose sur le lemme d'Osgood et un résultat d'analyse harmonique dû a R. Danchin [10].

Soit $\left(a^{i}, u^{i}, \nabla \Pi^{i}\right)$ pour $1 \leq i \leq 2$ deux solutions. Désignons par

$$
\left(\mathcal{M}^{i}, \delta \mathcal{M}\right) \stackrel{\text { déf }}{=}\left(\nabla u^{i}+{ }^{t} \nabla u^{i}, \mathcal{M}^{2}-\mathcal{M}^{1}\right)
$$

et

$$
(\delta a, \delta u, \nabla \delta \Pi) \stackrel{\text { déf }}{=}\left(a^{2}-a^{1}, u^{2}-u^{1}, \nabla \Pi^{2}-\nabla \Pi^{1}\right)
$$

leur différence, qui vérifie le système suivant

$$
\left\{\begin{array}{l}
\partial_{t} \delta a+u^{2} \cdot \nabla \delta a=-\delta u \cdot \nabla a^{1} \\
\partial_{t} \delta u+u^{2} \cdot \nabla \delta u-\mu^{1} \Delta \delta u+\nabla \delta \Pi=F\left(a^{i}, u^{i}, \nabla \Pi^{i}\right) \\
\operatorname{div} \delta u=0
\end{array}\right.
$$

où

$$
\begin{aligned}
F\left(a^{i}, u^{i}, \nabla \Pi^{i}\right) & =-\delta u \cdot \nabla u^{1}+a^{1}\left(\mu^{1} \Delta \delta u-\nabla \delta \Pi\right)+\delta a\left(\mu^{1} \Delta u^{2}-\nabla \Pi^{2}\right) \\
& +\operatorname{div}\left[\left(\widetilde{\mu}\left(a^{1}\right)-\mu^{1}\right) \delta \mathcal{M}\right]+\delta a \operatorname{div}\left[\left(\widetilde{\mu}\left(a^{2}\right)-\mu^{1}\right) \mathcal{M}^{2}\right] \\
& +a^{1} \operatorname{div}\left[\left(\widetilde{\mu}\left(a^{1}\right)-\mu^{1}\right) \delta \mathcal{M}\right]+\operatorname{div}\left[\left(\widetilde{\mu}\left(a^{2}\right)-\widetilde{\mu}\left(a^{1}\right)\right) \mathcal{M}^{2}\right] \\
& +a^{1} \operatorname{div}\left[\left(\widetilde{\mu}\left(a^{2}\right)-\widetilde{\mu}\left(a^{1}\right)\right) \mathcal{M}^{2}\right] .
\end{aligned}
$$

Donc $\delta a$ (resp. $(\delta u, \delta \nabla \Pi))$ vérifie l'équation de transport avec un terme de force $g$ (resp. le système de Stokes non stationnaire avec force $F$ ). Mais d'après deux résultats dûs à R. Danchin on peut contrôler $\delta a(\operatorname{resp} .(\delta u, \delta \nabla \Pi))$ dans $\dot{B}_{p r}^{s}$ pour $|s|<\frac{N}{p}+1$ (resp. pour $|s|<\frac{N}{p}$ ) par $\|g\|_{L_{T}^{1}\left(\dot{B}_{p r}^{s}\right)}$ (resp. $\|F\|_{\widetilde{L}_{T}^{1}\left(\dot{B}_{p r}^{s}\right)}$ ) (pour plus de précisions voir propositions 3.2 et 3.3 ).

Dans notre analyse nous allons distinguer deux cas : d'une part $3 \leq N$, $1 \leq p<N$ et d'autre part $N=2$ ou $N=p$ dans lesquels nous faisons appel à des lois de produit bien appropriées. 


\subsection{Cas où $3 \leq N$ et $1 \leq p<N$}

Proposition 3.1. Soient $\left(a^{1}, u^{1}, \nabla \Pi\right)$ et $\left(a^{2}, u^{2}, \nabla \Pi^{2}\right)$ deux solutions de $(\widetilde{\mathrm{INS}})$ avec mêmes données initiales $a_{0} \in \dot{B}_{p}^{\frac{N}{p}}\left(\mathbb{R}^{N}\right) \cap L^{\infty}\left(\mathbb{R}^{N}\right)$, u $u_{0}$ un champ de vecteurs à divergence nulle avec coefficients dans $\dot{B}_{p 1}^{\frac{N}{p}-1}\left(\mathbb{R}^{N}\right)$ et un terme de force extérieure $f \in\left(L_{\text {loc }}^{1}\left(\left[0, T^{*}\right) ; \dot{B}_{p 1}^{\frac{N}{p}-1}\left(\mathbb{R}^{N}\right)\right)\right)^{N}$ tel que $\mathcal{Q} f$ appartienne $\grave{a}\left(L_{l o c}^{1}\left(\left[0, T^{\star}\right) ; \dot{B}_{p 1}^{\frac{N}{p}-2}\left(\mathbb{R}^{N}\right)\right)\right)^{N}$. Supposons que pour $i=1,2$ on ait

$$
\begin{aligned}
a^{i} & \in C\left(\left[0, T^{\star}\right) ; \dot{B}_{p \infty}^{\frac{N}{p}}\right) \cap L^{\infty}\left(0, T^{\star} ; L^{\infty}\right), \\
u^{i} & \in\left(C\left(\left[0, T^{\star}\right) ; \dot{B}_{p 1}^{\frac{N}{p}-1}\right)\right)^{N} \cap\left(L_{L o c}^{1}\left(\left[0, T^{\star}\right) ; \dot{B}_{p 1}^{\frac{N}{p}+1}\right)\right)^{N}, \\
\nabla \Pi^{i} & \in\left(L_{L o c}^{1}\left(\left[0, T^{\star}\right) ; \dot{B}_{p 1}^{\frac{N}{p}-1}\right)\right)^{N} .
\end{aligned}
$$

Alors il existe une constante $c$ strictement positive telle que si on a

$$
\left\|a^{1}\right\|_{L_{T^{\star}}^{\infty}\left(\dot{B}_{p \infty}^{\frac{N}{p}} \cap L^{\infty}\right)} \leq c
$$

alors $\left(a^{2}, u^{2}, \nabla \Pi^{2}\right)=\left(a^{1}, u^{1}, \nabla \Pi^{1}\right)$.

Preuve. Tout d'abord on va montrer que $(\delta a, \delta u, \nabla \delta \Pi) \in F_{T}^{\frac{N}{p}-1}$, où $F_{T}^{\frac{N}{p}-1}=C\left([0, T] ; \dot{B}_{p \infty}^{\frac{N}{p}-1}\right) \times\left(L_{T}^{1}\left(\dot{B}_{p 1}^{\frac{N}{p}}\right) \cap C\left([0, T] ; \dot{B}_{p 1}^{\frac{N}{p}-2}\right)\right)^{N} \times\left(L_{T}^{1}\left(\dot{B}_{p 1}^{\frac{N}{p}-2}\right)\right)^{N}$ muni de la norme suivante

$$
\begin{gathered}
\|(a, u, \nabla \Pi)\|_{F_{T}^{\frac{N}{p}-1}} \stackrel{\text { déf }}{=}\|a\|_{L_{T}^{\infty}\left(\dot{B}_{p \infty}^{\frac{N}{p}-1}\right)}+\|u\|_{L_{T}^{\infty}\left(\dot{B}_{p 1}^{\frac{N}{p}-2}\right)}+\mu\left\|^{1}\right\| u \|_{L_{T}^{1}\left(\dot{B}_{p 1}^{\frac{N}{p}}\right)} \\
+\|\nabla \Pi\|_{L_{T}^{1}\left(\dot{B}_{p 1}^{\frac{N}{p}-2}\right)}
\end{gathered}
$$

Pour montrer que $(\delta a, \delta u, \nabla \delta \Pi) \in F_{T}^{\frac{N}{p}-1}$, il suffit de montrer que

$$
\left(a^{i}-a_{0}, \bar{u}^{i}, \nabla \bar{\Pi}^{i}\right) \in F_{T}^{\frac{N}{p}-1},
$$

où $\left(\bar{u}^{i}, \nabla \bar{\Pi}^{i}\right)$ sont définis par $u^{i}=u_{L}+\bar{u}^{i}$ et $\nabla \Pi^{i}=\nabla \Pi_{L}^{i}+\nabla \bar{\Pi}^{i}$, avec $\left(u_{L}, \nabla \Pi_{L}\right)$ la solution du système de Stokes non-stationnaire suivant

$$
\left\{\begin{array}{l}
\partial_{t} u_{L}-\mu^{1} \Delta u_{L}+\nabla \Pi_{L}=f \\
\operatorname{div} u_{L}=0 \\
u_{L \mid t=0}=u_{0}
\end{array}\right.
$$


La proposition 2.1 dans [5] assure que $u_{L}$ est à coefficients dans l'espace $C\left([0, T] ; \dot{B}_{p 1}^{\frac{N}{p}-1}\right) \cap L^{1}\left(0, T ; \dot{B}_{p 1}^{\frac{N}{p}+1}\right)$ et $\nabla \Pi_{L} \in\left(L^{1}\left(0, T ; \dot{B}_{p 1}^{\frac{N}{p}-1}\right)\right)^{N}$. De plus $\left(\bar{u}^{i}, \nabla \bar{\Pi}^{i}\right)$ vérifie

$$
\left(\mathrm{NS}_{\mathrm{mod}}\right)\left\{\begin{array}{l}
\partial_{t} \bar{u}^{i}-\mu^{1} \Delta \bar{u}^{i}+\nabla \bar{\Pi}^{i}=G\left(a^{i}, u^{i}, \nabla \Pi^{i}\right) \\
\operatorname{div} \bar{u}^{i}=0 \\
\bar{u}_{\mid t=0}^{i}=0,
\end{array}\right.
$$

où

$G\left(a^{i}, u^{i}, \nabla \Pi^{i}\right)=-u^{i} \cdot \nabla u^{i}+a^{i}\left(\mu^{1} \Delta u^{i}-\nabla \Pi^{i}\right)+\left(1+a^{i}\right) \operatorname{div}\left[\left(\widetilde{\mu}\left(a^{i}\right)-\mu^{1}\right) \mathcal{M}^{i}\right]$.

On applique à $\left(N S_{\text {mod }}\right)$ l'opérateur $\mathcal{P}$, on obtient

$$
\partial_{t} \bar{u}^{i}-\mu^{1} \Delta \bar{u}^{i}=\mathcal{P}\left(G\left(a^{i}, u^{i}, \nabla \Pi^{i}\right)\right)
$$

et on applique l'opérateur de divergence à $(\widetilde{I N S})$, on trouve

$$
\begin{aligned}
& \operatorname{div}\left(\left(1+a^{i}\right) \nabla \Pi^{i}\right)-\operatorname{div} \mathcal{Q} f= \\
& \quad \operatorname{div}\left\{-u^{i} \cdot \nabla u^{i}+\mu^{1}\left(a^{i} \Delta u^{i}\right)+\left(1+a^{i}\right) \operatorname{div}\left[\left(\widetilde{\mu}\left(a^{i}\right)-\mu^{1}\right) \mathcal{M}^{i}\right]\right\} .
\end{aligned}
$$

D'après l'inégalité $(2.10)$, on a $u^{i} \in\left(L_{T}^{2}\left(\dot{B}_{p 1}^{\frac{N}{p}}\right)\right)^{N}$, et comme $N>p, N \geq 3$ alors l'inégalité $(2.5)$ nous conduit à $u^{i} \cdot \nabla u^{i} \in\left(L_{T}^{2}\left(\dot{B}_{p 1}^{\frac{N}{p}-2}\right)\right)^{N}$. Par ailleurs en utilisant les inégalités $(2.10)$ et $(2.5)$, on obtient $a^{i} \Delta u^{i} \in\left(L_{T}^{2}\left(\dot{B}_{p 1}^{\frac{N}{p}-2}\right)\right)^{N}$. Dans la suite on utilise le fait que

$$
\left\|a^{i}\right\|_{L^{\infty}} \leq\left\|a_{0}\right\|_{L^{\infty}} .
$$

Les inégalités (2.5), (2.17) et l'inégalité de Bernstein impliquent

$$
\begin{aligned}
\|\left(1+a^{i}\right) \operatorname{div}[ & \left.\left.\left(\widetilde{\mu}\left(a^{i}\right)-\mu^{1}\right) \mathcal{M}^{i}\right] \|_{L_{T}^{2}\left(\dot{B}_{p 1}^{\frac{N}{p}}-2\right.}\right) \\
& \left.\lesssim\left(1+\left\|a^{i}\right\|_{L_{T}^{\infty}\left(\dot{B}_{p \infty}^{\frac{N}{p}} \cap L^{\infty}\right)}\right)\left\|\left(\widetilde{\mu}\left(a^{i}\right)-\mu^{1}\right) \mathcal{M}^{i}\right\|_{L_{T}^{2}\left(\dot{B}_{p 1}^{\frac{N}{p}}-1\right.}\right) \\
& \lesssim\left(1+\left\|a^{i}\right\|_{L_{T}^{\infty}\left(\dot{B}_{p}^{\frac{N}{p}} \cap L^{\infty}\right)}\right)\left\|\widetilde{\mu}\left(a^{i}\right)-\mu^{1}\right\|_{L_{T}^{\infty}\left(\dot{B}_{p \infty}^{p} \cap L^{\infty}\right)}\left\|u^{i}\right\|_{L_{T}^{2}\left(\dot{B}_{p 1}^{\frac{N}{p}}\right)} \\
& \lesssim\left(1+\left\|a^{i}\right\|_{L_{T}^{\infty}\left(\dot{B}_{p \infty}^{\frac{N}{p}} \cap L^{\infty}\right)}\right)\left\|a^{i}\right\|_{L_{T}^{\infty}\left(\dot{B}_{p \infty}^{\frac{N}{p}} \cap L^{\infty}\right)}\left\|u^{i}\right\|_{L_{T}^{2}\left(\dot{B}_{p 1}^{\frac{N}{p}}\right)}
\end{aligned}
$$

Donc la quantité de gauche de l'égalité (3.2) appartient à $L_{T}^{2}\left(\dot{B}_{p 1}^{\frac{N}{p}-3}\right)$. Sous l'hypothese $\mathcal{Q} f \in\left(L_{l o c}^{1}\left(\left[0, T^{\star}\right) ; \dot{B}_{p 1}^{\frac{N}{p}-2}\right)\right)^{N}$ et le fait que $\left\|a^{i}\right\|_{L_{T}^{\infty}\left(\dot{B}_{p \infty}^{\frac{N}{p}} \cap L^{\infty}\right)}$ est 
petit, on utilise les inégalités $(2.5)$ et $(3.2)$, on obtient $\nabla \Pi^{i} \in\left(L_{T}^{1}\left(\dot{B}_{p 1}^{\frac{N}{p}-2}\right)\right)^{N}$ dès que $T<+\infty$. D'où l'inégalité $(2.5)$ montre que $a^{i} \nabla \Pi^{i} \in\left(L_{T}^{1}\left(\dot{B}_{p 1}^{\frac{N}{p}-2}\right)\right)^{N}$. Et par suite pour $T<\infty$, on trouve que $G\left(a^{i}, u^{i}, \nabla \Pi^{i}\right) \in\left(L_{T}^{1}\left(\dot{B}_{p 1}^{\frac{N}{p}-2}\right)\right)^{N}$. Puisque l'opérateur $\mathcal{P}$ est continu sur $\dot{B}_{p r}^{s}$ pour tout $s \in \mathbb{R}$ et $(p, r) \in[1, \infty]^{2}$, alors la quantité de gauche de l'égalité (3.1) appartient à $\left(L_{T}^{1}\left(\dot{B}_{p 1}^{\frac{N}{p}-2}\right)\right)^{N}$. De nouveau la proposition 2.1 dans [5], implique $\bar{u}^{i}$ appartient à

$$
\left(L_{T}^{1}\left(\dot{B}_{p 1}^{\frac{N}{p}}\right)\right)^{N} \cap\left(C\left([0, T] ; \dot{B}_{p 1}^{\frac{N}{p}-2}\right)\right)^{N} .
$$

De même, on a $\nabla \bar{\Pi}^{i} \in\left(L_{T}^{1}\left(\dot{B}_{p 1}^{\frac{N}{p}-2}\right)\right)^{N}$. Mais $\partial_{t} a^{i}=-u^{i} \cdot \nabla a^{i}$, donc grâce à l'inégalité $(2.5)$, on a $\partial_{t} a^{i} \in L_{T}^{2}\left(\dot{B}_{p \infty}^{\frac{N}{p}-1}\right)$ et par suite l'inégalité de CauchySchwarz implique que $\left(a^{i}-a_{0}\right) \in C\left([0, T] ; \dot{B}_{p \infty}^{\frac{N}{p}-1}\right)$. Par ailleurs $\delta a=\left(a^{2}-a_{0}\right)$ $-\left(a^{1}-a_{0}\right), \delta u=\bar{u}^{2}-\bar{u}^{1}$ et $\nabla \delta \Pi=\nabla \bar{\Pi}^{2}-\nabla \bar{\Pi}^{1}$, donc $(\delta a, \delta u, \nabla \delta \Pi) \in F_{T}^{\frac{N}{p}-1}$.

Pour estimer $(\delta a, \delta u, \nabla \delta \Pi)$ nous allons utiliser les propositions suivantes, qui nous donnent une estimation de la solution de l'équation de transport et de celle de l'équation de Stokes non stationnaire linéaire. Admettons-les pour le moment.

Proposition 3.2. Soient $r \in[1,+\infty], p \in[1,+\infty]$ et $s \in \mathbb{R}$ tel que $|s|<$ $1+\frac{N}{p}$. Soit $v$ un champ de vecteurs à divergence nulle tel que $\nabla v$ soit $\grave{a}$ coefficients dans $L_{T}^{1}\left(\dot{B}_{p r}^{\frac{N}{p}} \cap L^{\infty}\right)$. Supposons que $f_{0} \in \dot{B}_{p r}^{s}, F \in L_{T}^{1}\left(\dot{B}_{p r}^{s}\right)$. Soit $f \in L_{T}^{\infty}\left(\dot{B}_{p r}^{s}\right) \cap C\left([0, T] ; \mathcal{S}^{\prime}\right)$ une solution du système suivant

$$
\left\{\begin{array}{l}
\partial_{t} f+\operatorname{div}(v f)=F \\
f_{\mid t=0}=f_{0}
\end{array}\right.
$$

Alors il existe une constante $C$ strictement positive dépendant de $N$ et $s$ telle que

$$
\|f\|_{\tilde{L}_{T}^{\infty}\left(\dot{B}_{p r}^{s}\right)} \leq e^{C V(t)}\left(\left\|f_{0}\right\|_{\dot{B}_{p r}^{s}}+\int_{0}^{t} e^{-C V(\tau)}\|F(\tau)\|_{\dot{B}_{p r}^{s}} d \tau\right),
$$

où

$$
V(t)=\int_{0}^{t}\|\nabla v(\tau)\|_{\dot{B}_{p}^{\frac{N}{p}} \cap L^{\infty}} d \tau
$$

De plus $f \in C\left([0, T] ; \dot{B}_{p r}^{s}\right)$ si $r<\infty$. 
Proposition 3.3. Soient s tel que $|s|<\frac{N}{p}$ si $2 \leq p<\infty,-\frac{N}{p^{\prime}}<s<\frac{N}{p}$ si $1<p \leq 2, r \in[1,+\infty]$, $u_{0}$ un champ de vecteurs à divergence nulle avec coefficients dans $\dot{B}_{p r}^{s}$ et $g$ un champ de vecteurs à coefficients dans $\widetilde{L}_{T}^{1}\left(\dot{B}_{p r}^{s}\right)$. Soient $u$ et $v$ deux champs de vecteurs à divergence nulle tels que $\nabla v$ soit à coefficients dans $L_{T}^{1}\left(\dot{B}_{p r}^{\frac{N}{p}} \cap L^{\infty}\right)$ (resp. $\left.L_{T}^{1}\left(\dot{B}_{p 1}^{\frac{N}{p}}\right)\right)$ et $u \in\left(C\left(\left[0, T ; \dot{B}_{p r}^{s}\right)\right)^{N} \cap\right.$ $\left(\widetilde{L}_{T}^{1}\left(\dot{B}_{p r}^{s+2}\right)\right)^{N}$ soit une solution du système de Stokes non stationnaire

$$
(\mathrm{L}) \quad\left\{\begin{array}{l}
\partial_{t} u+v \cdot \nabla u-\nu \Delta u+\nabla \Pi=g \\
\operatorname{div} u=0 \\
u_{\mid t=0}=u_{0} .
\end{array}\right.
$$

Alors il existe $C$ dépendant de $N$ et s tel que u vérifie l'estimation suivante

$$
\begin{aligned}
& \|u\|_{\widetilde{L}_{T}^{\infty}\left(\dot{B}_{p r}^{s}\right)}+\nu\|u\|_{\widetilde{L}_{T}^{1}\left(\dot{B}_{p r}^{s+2}\right)}+\|\nabla \Pi\|_{\widetilde{L}_{T}^{1}\left(\dot{B}_{p r}^{s}\right)} \\
& \leq \exp \left(C\|\nabla v\|_{L_{T}^{1}\left(\dot{B}_{p r}^{N} L^{\infty}\right.}\right)\left\{\left\|u_{0}\right\|_{\dot{B}_{p r}^{s}}+C\|g\|_{\widetilde{L}_{T}^{1}\left(\dot{B}_{p r}^{s}\right)}\right\} .
\end{aligned}
$$

On a de plus l'estimation suivante:

$$
\begin{aligned}
& \|u\|_{\widetilde{L}_{T}^{\infty}\left(\dot{B}_{p \infty}^{\left.-\frac{N}{p}\right)}\right.}+\nu\|u\|_{\widetilde{L}_{T}^{1}\left(\dot{B}_{p \infty}^{2-\frac{N}{p}}\right)}+\|\nabla \Pi\|_{\widetilde{L}_{T}^{1}\left(\dot{B}_{p}^{-\frac{N}{p}}\right)} \\
& \quad \leq \exp \left(C\|\nabla v\|_{L_{T}^{1}\left(\dot{B}_{p 1}^{\frac{N}{p}}\right)}\right)\left\{\left\|u_{0}\right\|_{\dot{B}_{p \infty}^{-\frac{N}{p}}}+C\|g\|_{\widetilde{L}_{T}^{1}\left(\dot{B}_{p}^{-\frac{N}{p}}\right)}\right\} .
\end{aligned}
$$

Comme conséquence des propositions 3.2 et 3.3 , on a la majoration suivante pour tout $t \leq T$

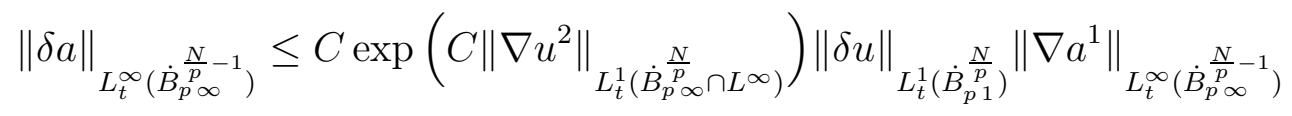

et

$$
\begin{aligned}
& \|\delta u\|_{L_{t}^{\infty}\left(\dot{B}_{p 1}^{\frac{N}{p}}-2\right)}+\mu^{1}\|\delta u\|_{L_{t}^{1}\left(\dot{B}_{p 1}^{\frac{N}{p}}\right)}+\|\nabla \delta \Pi\|_{L_{t}^{1}\left(\dot{B}_{p 1}^{\frac{N}{p}}-2\right.} \\
& \lesssim \exp \left(C\left\|\nabla u^{2}\right\|_{L_{t}^{1}\left(\dot{B}_{p 1}^{\frac{N}{p}}\right)}\right)\left\|F\left(a^{i}, u^{i}, \nabla \Pi^{i}\right)\right\|_{L_{t}^{1}\left(\dot{B}_{p 1}^{\frac{N}{p}-2}\right)} .
\end{aligned}
$$

Tout d'abord les inégalités (2.4) et (2.5), donnent du fait que $3 \leq N$ et $p<N$,

$$
\begin{aligned}
& \left.\left\|-\delta u \cdot \nabla u^{1}+a^{1}\left(\mu^{1} \Delta \delta u-\nabla \delta \Pi\right)+\delta a\left(\mu^{1} \Delta u^{2}-\nabla \Pi^{2}\right)\right\|_{L_{T}^{1}\left(\dot{B}_{p 1}^{\frac{N}{p}}-2\right.}\right) \\
& \lesssim\|\delta u\|_{L_{T}^{\infty}\left(\dot{B}_{p 1}^{\frac{N}{p}-2}\right)}\left\|\nabla u^{1}\right\|_{L_{T}^{1}\left(\dot{B}_{p 1}^{\frac{N}{p}}\right)}+\left(\|\delta u\|_{L_{T}^{1}\left(\dot{B_{p 1}}\right)}+\|\nabla \delta \Pi\|_{L_{T}^{\frac{N}{p}\left(\dot{B}_{p 1}^{\frac{N}{p}-2}\right)}}\right) \\
& \times\left\|a^{1}\right\|_{L_{T}^{\infty}\left(\dot{B}_{p}^{\frac{N}{p}} \cap L^{\infty}\right)}+\|\delta a\|_{L_{T}^{\infty}\left(\dot{B}_{p \infty}^{\frac{N}{p}-1}\right)}\left(\left\|\Delta u^{2}\right\|_{L_{T}^{1}\left(\dot{B}_{p 1}^{\frac{N}{p}-1}\right)}+\left\|\nabla \Pi^{2}\right\|_{L_{T}^{1}\left(\dot{B}_{p 1}^{\frac{N}{p}-1}\right)}\right) .
\end{aligned}
$$


D'après la formule de Taylor avec reste intégral et le fait que $\left\|a^{i}\right\|_{L^{\infty}}<<1$, on trouve

$$
\left\|\widetilde{\mu}\left(a^{i}\right)-\mu^{1}\right\|_{L^{\infty}} \lesssim\left\|a^{i}\right\|_{L^{\infty}}
$$

De même d'après les inégalités (2.5) et (2.17), on a

$$
\begin{aligned}
\left\|\operatorname{div}\left[\left(\widetilde{\mu}\left(a^{1}\right)-\mu^{1}\right) \delta \mathcal{M}\right]\right\|_{L_{T}^{1}\left(\dot{B}_{p 1}^{\frac{N}{p}-2}\right)} & \lesssim\left\|\widetilde{\mu}\left(a^{1}\right)-\mu^{1}\right\|_{L_{T}^{\infty}\left(\dot{B}_{p \infty}^{\frac{N}{p}} \cap L^{\infty}\right)}\|\delta u\|_{L_{T}^{1}\left(\dot{B}_{p 1}^{\frac{N}{p}}\right)} \\
& \lesssim\left\|a^{1}\right\|_{L_{T}^{\infty}\left(\dot{B}_{p}^{\frac{N}{p}} \cap L^{\infty}\right)}\|\delta u\|_{L_{T}^{1}\left(\dot{B}_{p 1}^{\frac{N}{p}}\right)} .
\end{aligned}
$$

Comme $p<N$ et $3 \leq N$, alors les inégalités (2.5) et (2.17) impliquent

$$
\begin{aligned}
\| a^{1} \operatorname{div}\left[\left(\widetilde{\mu}\left(a^{1}\right)\right.\right. & \left.\left.-\mu^{1}\right) \delta \mathcal{M}\right] \|_{L_{T}^{1}\left(\dot{B}_{p 1}^{\frac{N}{p}}-2\right)} \\
& \lesssim\left\|a^{1}\right\|_{L_{T}^{\infty}\left(\dot{B}_{p}^{\frac{N}{p}} \cap L^{\infty}\right)}\left\|\left(\widetilde{\mu}\left(a^{1}\right)-\mu^{1}\right) \delta \mathcal{M}\right\|_{L_{T}^{1}\left(\dot{B}_{p 1}^{\frac{N}{p}}-1\right)} \|\left(\widetilde{\mu}\left(a^{1}\right)-\mu^{1}\left\|_{L_{T}^{\infty}\left(\dot{B}_{p \infty}^{\frac{N}{p}} \cap L^{\infty}\right)}\right\| \nabla \delta u \|_{L_{T}^{1}\left(\dot{B}_{p 1}^{\frac{N}{p}}-1\right)}\right. \\
& \lesssim\left\|a^{1}\right\|_{L_{T}^{\infty}\left(\dot{B}_{p \infty}^{\frac{N}{p}} \cap L^{\infty}\right)}\|\| \|_{L_{T}^{1}\left(\dot{B}_{p 1}^{\frac{N}{p}}\right)} \\
& \lesssim\left\|a^{1}\right\|_{L_{T}^{\infty}\left(\dot{B}_{p}^{p} \cap L^{\infty}\right)}^{2}
\end{aligned}
$$

D'après les inégalités de Bernstein (2.11) et (3.10), il existe $0<T_{2} \leq T$ tel que

$$
\begin{aligned}
\left\|\left(\widetilde{\mu}\left(a^{2}\right)-\widetilde{\mu}\left(a^{1}\right)\right) \mathcal{M}^{2}\right\|_{L_{T_{2}}^{1}\left(\dot{B}_{p 1}^{\frac{N}{p}}-1\right)} \lesssim \int_{0}^{T_{2}}\left\|\widetilde{\mu}\left(a^{2}\right)-\widetilde{\mu}\left(a^{1}\right)\right\|_{\dot{B}_{p 1}^{\frac{N}{p}-1}}\left\|\nabla u^{2}\right\|_{\dot{B}_{p 1}^{\frac{N}{p}}} d t \\
\quad \lesssim \int_{0}^{T_{2}}\|\delta a\|_{\dot{B}_{p \infty}^{\frac{N}{p}-1}}\left(1-\log \|\delta a\|_{\dot{B}_{p \infty}^{\frac{N}{p}-1}}\right)\left\|u^{2}\right\|_{\dot{B}_{p 1}^{\frac{N}{p}+1}} d t .
\end{aligned}
$$

Grâce au lemme 2.4 on démontre que la quantité $a^{1} \operatorname{div}\left[\left\{\widetilde{\mu}\left(a^{2}\right)-\widetilde{\mu}\left(a^{1}\right)\right\} \mathcal{M}^{2}\right]$ est dans $L_{T}^{1}\left(\dot{B}_{p 1}^{\frac{N}{p}-2}\right)$ est contrôlée par la norme $L^{1}$ par rapport au temps de $\|\delta a\|_{\dot{B}_{p 1}^{\frac{N}{p}-1}}\left(1-\log \|\delta a\|_{\dot{B}_{p 1}^{\frac{N}{p}-1}}\right)$.

Lemme 3.1. Soient $p<N, 3 \leq N$ et $\left(a^{1}, a^{2}, u^{2}\right)$ vérifiant les conditions de la proposition 3.1 . Alors il existe $T_{2}$ strictement positif dépendant de $a_{0}$ et $u_{0}$, tel que pour $0 \leq T \leq T_{2}$

$$
\begin{aligned}
& \left\|a^{1} \operatorname{div}\left[\left\{\widetilde{\mu}\left(a^{2}\right)-\widetilde{\mu}\left(a^{1}\right)\right\} \mathcal{M}^{2}\right]\right\|_{L_{T}^{1}\left(\dot{B}_{p 1}^{\frac{N}{p}-2}\right)} \\
& \quad \leq C_{T_{2}} \int_{0}^{T}\|\delta a\|_{\dot{B}_{p \infty}^{\frac{N}{p}-1}}\left(1-\log \|\delta a\|_{\dot{B}_{p \infty}^{\frac{N}{p}-1}}\right)\left\|u^{2}\right\|_{\dot{B}_{p 1}^{\frac{N}{p}+1}} d t .
\end{aligned}
$$


En suivant la même démonstration dans le lemme 3.1, on obtient le résultat suivant.

Lemme 3.2. Soient $1 \leq p<N, 3 \leq N$ et $\left(a^{2}, u^{2}\right)$ vérifiant les conditions de la proposition 3.1. Alors il existe $T_{3}$ strictement positif dépendant de $a_{0}$ et $u_{0}$, tel que, pour $0 \leq T \leq T_{3}$,

$$
\begin{aligned}
\left\|\delta a \operatorname{div}\left[\left\{\widetilde{\mu}\left(a^{2}\right)-\mu^{1}\right\} \mathcal{M}^{2}\right]\right\|_{L_{T}^{1}\left(\dot{B}_{p 1}^{\frac{N}{p}-2}\right)} & \leq C_{T_{3}} \int_{0}^{T}\|\delta a(t)\|_{\dot{B}_{p \infty}^{\frac{N}{p}-1}} \\
& \times\left(1-\log \|\delta a(t)\|_{\dot{B}_{p \infty}^{\frac{N}{p}-1}}\right)\left\|u^{2}\right\|_{\dot{B}_{p 1}^{\frac{N}{p}+1}} d t .
\end{aligned}
$$

Donc grâce aux inégalités précedentes et aux lemmes 3.1 et 3.2, il existe $T_{1}$ strictement positif tel que

$$
\begin{aligned}
& \left.\left\|F\left(a^{i}, u^{i}, \nabla \Pi^{i}\right)\right\|_{L_{T_{1}}^{1}\left(\dot{B}_{p 1}^{\frac{N}{p}-2}\right)} \lesssim\|\delta u\|_{L_{T_{1}}^{\infty}\left(\dot{B}_{p 1}^{\frac{N}{p}-2}\right)}\left\|u^{1}\right\|_{L_{T_{1}}^{1}\left(\dot{B}_{p 1}^{\frac{N}{p}}+1\right.}\right) \\
& +\left\|a^{1}\right\|_{L_{T_{1}}^{\infty}\left(\dot{B}_{p}^{p} \cap L^{\infty}\right)}\left(\|\delta u\|_{L_{T_{1}}^{1}\left(\dot{B}_{p 1}^{\frac{N}{p}}\right)}+\|\nabla \delta \Pi\|_{L_{T_{1}}^{1}\left(\dot{B}_{p 1}^{\frac{N}{p}}-2\right.}\right)
\end{aligned}
$$

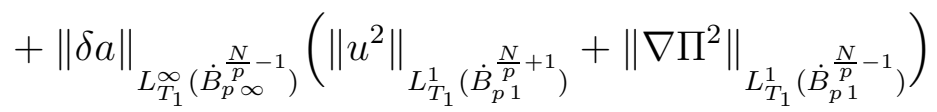

$$
\begin{aligned}
& +\left(\left\|a^{1}\right\|_{L_{T_{1}}^{\infty}\left(\dot{B}_{p}^{\frac{N}{p}} \cap L^{\infty}\right)}+\left\|a^{1}\right\|_{L_{T_{1}}^{\infty}\left(\dot{B}_{p \infty}^{\frac{N}{p}} \cap L^{\infty}\right)}^{2}\right) \mu^{1}\|\delta u\|_{L_{T_{1}}^{1}\left(\dot{B}_{p 1}^{\frac{N}{p}}\right)} \\
& +\int_{0}^{T_{1}}\|\delta a(t)\|_{\dot{B}_{p \infty}^{\frac{N}{p}-1}}\left(1-\log \|\delta a(t)\|_{\dot{B}_{p \infty}^{\frac{N}{p}-1}}\right)\left\|u^{2}\right\|_{\dot{B}_{p 1}^{\frac{N}{p}+1}} d t .
\end{aligned}
$$

De plus, quitte à diminuer $T_{1}$ on peut supposer que

$$
\exp \left\{C\left\|\nabla u^{2}\right\|_{L_{T_{1}}^{1}\left(\dot{B}_{p 1}^{\frac{N}{p}}\right)}\right\} \leq 2 \text { et } \mu^{1} \sum_{i=1}^{2}\left\|u_{L_{T_{1}}^{i}\left(\dot{B}_{p 1}^{\frac{N}{p}+1}\right)}+\right\| \nabla \Pi^{2} \|_{L_{T_{1}}^{1}\left(\dot{B}_{p 1}^{\frac{N}{p}-1}\right)} \leq c .
$$

En utilisant le fait que $\left\|a^{1}\right\|_{L_{T}^{\infty}\left(\dot{B}_{p \infty}^{\frac{N}{p}} \cap L^{\infty}\right)} \leq c<1$, on obtient

$$
\begin{aligned}
& \left\|F\left(a^{i}, u^{i}, \nabla \Pi^{i}\right)\right\|_{L_{T_{1}}^{1}\left(\dot{B}_{p 1}^{\frac{N}{p}-2}\right)} \lesssim c\left(\|\delta u\|_{L_{T_{1}}^{\infty}\left(\dot{B}_{p 1}^{\frac{N}{p}-2}\right)}+\|\delta u\|_{L_{T_{1}}^{1}\left(\dot{B}_{p 1}^{p}\right)}\right. \\
& \left.+\|\nabla \delta \Pi\|_{L_{T_{1}}^{1}\left(\dot{B}_{p 1}^{\frac{N}{p}-2}\right)}+\|\delta a\|_{L_{T_{1}}^{\infty}\left(\dot{B}_{p \infty}^{\frac{N}{p}-1}\right)}\right) \\
& +\int_{0}^{T_{1}}\|\delta a\|_{\dot{B}_{p \infty}^{\frac{N}{p}-1}}\left(1-\log \|\delta a\|_{\dot{B}_{p \infty}^{\frac{N}{p}-1}}\right)\left\|u^{2}\right\|_{\dot{B}_{p 1}^{\frac{N}{p}+1}} d t .
\end{aligned}
$$

Ainsi

$$
\|(\delta a, \delta u, \delta \nabla \Pi)\|_{F_{T_{1}}^{\frac{N}{p}-1}} \leq C \int_{0}^{T_{1}}\|\delta a\|_{\dot{B}_{p \infty}^{\frac{N}{p}-1}}\left(1-\log \|\delta a\|_{\dot{B}_{p \infty}^{\frac{N}{p}-1}}\right)\left\|u^{2}\right\|_{\dot{B}_{p 1}^{\frac{N}{p}+1}} d t .
$$


On utilisant de nouveau le fait que la fonction $x \longmapsto x(1-\log x)$ est croissante sur $] 0,1]$, on trouve

$$
\begin{aligned}
& \|(\delta a, \delta u, \delta \nabla \Pi)\|_{F_{T_{1}}^{\frac{N}{p}-1}} \\
& \lesssim \int_{0}^{T_{1}}\|(\delta a, \delta u, \delta \nabla \Pi)\|_{F_{t}^{\frac{N}{p}-1}}\left(1-\log \|(\delta a, \delta u, \delta \nabla \Pi)\|_{F_{t}^{\frac{N}{p}-1}}\right)\left\|u^{2}\right\|_{\dot{B}_{p 1}^{\frac{N}{p}+1}} d t .
\end{aligned}
$$

Or

$$
\int_{0}^{1} \frac{d r}{r(1-\log r)} d r=+\infty
$$

et $t \mapsto\left\|u^{2}\right\|_{\dot{B}_{p 1}^{\frac{N}{p}+1}}$ est localement intégrable, donc le lemme d'Osgood (voir par exemple [4]) implique que $(\delta a, \delta u, \delta \nabla \Pi)=0$ sur $\left[0, T_{1}\right]$ pour $T_{1}$ petit. Un argument standard de connexité permet d'obtenir $(\delta a, \delta u, \delta \nabla \Pi)=0$ sur $[0, T]$. Ainsi la démonstration est achevée pour $1<p<N$ et $3 \leq N$. Les calculs qu'on a effectués sont valables pour $1<p$ (car ils reposent sur la proposition 3.3). Le cas $p=1$ s'en déduit par injection.

\subsection{Si $N=p$ ou $N=2$}

Dans le cas limite $N=p$ ou $N=2$ la condition $a^{1}$ est petit dans

$$
L_{T^{\star}}^{\infty}\left(\dot{B}_{p \infty}^{\frac{N}{p}} \cap L^{\infty}\right)
$$

est insuffisante pour boucler les estimations. Mais on a le résultat suivant :

Proposition 3.4. Soient $\left(a^{1}, u^{1}, \nabla \Pi\right)$ et $\left(a^{2}, u^{2}, \nabla \Pi^{2}\right)$ deux solutions de $(\widetilde{\mathrm{INS}})$ avec donnée initiale $a_{0} \in \dot{B}_{N 1}^{1}, u_{0} \in\left(\dot{B}_{N 1}^{0}\left(\mathbb{R}^{N}\right)\right)^{N}$ avec $\operatorname{div} u_{0}=0$ et $f$ à coefficients dans $L_{\text {loc }}^{1}\left(\left[0, T^{*}\right) ; \dot{B}_{N 1}^{0}\left(\mathbb{R}^{N}\right)\right)$ tel que $\mathcal{Q} f$ à coefficients dans $L_{\text {loc }}^{1}\left(\left[0, T^{\star}\right) ; \dot{B}_{N 1}^{-1}\left(\mathbb{R}^{N}\right)\right)$. Supposons que pour $i=1,2$ on ait

$$
\begin{aligned}
a^{i} & \in C\left(\left[0, T^{\star}\right) ; \mathcal{S}^{\prime}\right) \cap L_{l o c}^{\infty}\left(\left[0, T^{\star}\right) ; \dot{B}_{N 1}^{1}\right), \\
u^{i} & \in\left(C\left(\left[0, T^{\star}\right) ; \dot{B}_{N 1}^{0}\right)\right)^{N} \cap\left(L_{l o c}^{1}\left(\left[0, T^{\star}\right) ; \dot{B}_{N 1}^{2}\right)\right)^{N}, \\
\nabla \Pi^{i} & \in\left(L_{l o c}^{1}\left(\left[0, T^{\star}\right) ; \dot{B}_{N 1}^{0}\right)\right)^{N} .
\end{aligned}
$$

Alors il existe une constante $c$ strictement positive telle que si on a

$$
\left\|a^{1}\right\|_{\widetilde{L}_{T^{\star}}^{\infty}\left(\dot{B}_{N 1}^{1}\right)} \leq c
$$

alors $\left(a^{2}, u^{2}, \nabla \Pi^{2}\right)=\left(a^{1}, u^{1}, \nabla \Pi^{1}\right)$. 
Preuve. On va montrer tout d'abord que $(\delta a, \delta u, \nabla \delta \Pi) \in G_{T}^{0}$, où $G_{T}^{0}=C\left([0, T] ; \dot{B}_{N \infty}^{0}\right) \times\left(\widetilde{L}_{T}^{1}\left(\dot{B}_{N \infty}^{1}\right) \cap C\left([0, T] ; \dot{B}_{N \infty}^{-1}\right)\right)^{N} \times\left(\widetilde{L}_{T}^{1}\left(\dot{B}_{N \infty}^{-1}\right)\right)^{N}$, muni de la norme suivante

$$
\begin{aligned}
\|(a, u, \nabla \Pi)\|_{G_{T}^{0}} \stackrel{\text { déf }}{=}\|a\|_{L_{T}^{\infty}\left(\dot{B}_{N \infty}^{0}\right)}+\|u\|_{L_{T}^{\infty}\left(\dot{B}_{N \infty}^{-1}\right)} & \left.+\mu^{1}\|u\|_{\widetilde{L}_{T}^{1}\left(\dot{B}_{N \infty}^{1}\right)}\right) \\
& +\|\nabla \Pi\|_{\widetilde{L}_{T}^{1}\left(\dot{B}_{N \infty}^{-1}\right)} .
\end{aligned}
$$

Le seule différence avec la preuve précédente est dans le traitement des produits du type $a^{i} \nabla \Pi^{i}$. Pour cela, on utilise l'inégalité (2.6), qui assure que le membre de droite de l'égalité (3.1) appartient à $\left(L_{T}^{2}\left(\dot{B}_{N \infty}^{-1}\right)\right)^{N}$, et par suite l'inégalité de Cauchy-Schwarz implique que cette quantité appartient à $\widetilde{L}_{T}^{1}\left(\dot{B}_{N \infty}^{-1}\right)$ pour $T<\infty$. Donc la proposition 2.1 dans [5] implique que $(\delta a, \delta u, \nabla \delta \Pi) \in G_{T}^{0}$.

En suivant la même démarche qui conduit à montrer que $(\delta a, \delta u, \nabla \delta \Pi)=0$ sur $[0, T]$ pour $1 \leq p<N$ et $3<N$, on obtient grâce aux propositions 3.2 et 3.3 les estimations suivantes:

$$
\|\delta a\|_{L_{t}^{\infty}\left(\dot{B}_{N \infty}^{0}\right)} \leq \exp \left(C\left\|\nabla u^{2}\right\|_{L_{t}^{1}\left(\dot{B}_{N 1}^{1}\right)}\right)\left\|\delta u \cdot \nabla a^{1}\right\|_{L_{t}^{1}\left(\dot{B}_{N \infty}^{0}\right)}
$$

et

$$
\begin{aligned}
& \|\delta u\|_{L_{t}^{\infty}\left(\dot{B}_{N \infty}^{-1}\right)}+\mu^{1}\|\delta u\|_{\widetilde{L}_{t}^{1}\left(\dot{B}_{N \infty}^{1}\right)}+\|\nabla \delta \Pi\|_{\widetilde{L}_{t}^{1}\left(\dot{B}_{N \infty}^{-1}\right)} \\
& \leq C \exp \left(C\left\|u^{2}\right\|_{L_{t}^{1}\left(\dot{B}_{N 1}^{2}\right)}\right)\left\|F\left(a^{i}, u^{i}, \nabla \Pi^{i}\right)\right\|_{\widetilde{L}_{t}^{1}\left(\dot{B}_{N \infty}^{-1}\right)} .
\end{aligned}
$$

D'après les inégalités (2.5), de Bernstein, de Minkowski et (2.19), on a

$$
\left\|\delta u \cdot \nabla a^{1}\right\|_{L_{t}^{1}\left(\dot{B}_{N \infty}^{0}\right)} \lesssim\|\delta u\|_{L_{t}^{1}\left(\dot{B}_{N 1}^{1}\right)}\left\|a^{1}\right\|_{\widetilde{L}_{t}^{\infty}\left(\dot{B}_{N 1}^{1}\right)}
$$

et

$$
\|\delta u\|_{L_{t}^{1}\left(\dot{B}_{N 1}^{1}\right)} \lesssim\|\delta u\|_{\widetilde{L}_{t}^{1}\left(\dot{B}_{N \infty}^{1}\right)} \log \left(e+\frac{\|\delta u\|_{\widetilde{L}_{t}^{1}\left(\dot{B}_{N \infty}^{0}\right)}+\|\delta u\|_{\widetilde{L}_{t}^{1}\left(\dot{B}_{N \infty}^{2}\right)}}{\left.\|\delta u\|_{\widetilde{L}_{t}^{1}\left(\dot{B}_{N \infty}^{1}\right)}\right)} .\right.
$$

Mais les inégalités de Hölder et de Minkowski impliquent que

$$
\|\delta u\|_{\widetilde{L}_{t}^{1}\left(\dot{B}_{N \infty}^{0}\right)} \leq t \sum_{i=1}^{2}\left\|u^{i}\right\|_{L_{t}^{\infty}\left(\dot{B}_{N 1}^{0}\right)} \quad \text { et } \quad\|\delta u\|_{\widetilde{L}_{t}^{1}\left(\dot{B}_{N \infty}^{2}\right)} \leq \sum_{i=1}^{2}\left\|u^{i}\right\|_{L_{t}^{1}\left(\dot{B}_{N 1}^{2}\right)} .
$$

Et par suite

$$
\begin{aligned}
\|\delta a\|_{L_{t}^{\infty}\left(\dot{B}_{N \infty}^{0}\right)} & \left.\leq \exp \left(C\left\|\nabla u^{2}\right\|_{L_{t}^{1}\left(\dot{B}_{N 1}^{1}\right)}\right)\left\|a^{1}\right\|_{\tilde{L}_{t}^{\infty}\left(\dot{B}_{N 1}^{1}\right)}\|\delta u\|_{\widetilde{L}_{t}^{1}\left(\dot{B}_{N \infty}^{1}\right)}\right) \\
& \times \log \left(e+\frac{\sum_{i=1}^{2}\left(t\left\|u^{i}\right\|_{L_{t}^{\infty}\left(\dot{B}_{N 1}^{0}\right)}+\left\|u^{i}\right\|_{L_{t}^{1}\left(\dot{B}_{N 1}^{2}\right)}\right)}{\left.\|\delta u\|_{\widetilde{L}_{t}^{1}\left(\dot{B}_{N \infty}^{1}\right)}\right)}\right) .
\end{aligned}
$$


Aussi les inégalités (2.13) et de Bernstein impliquent

$$
\begin{aligned}
& \left\|-\delta u \cdot \nabla u^{1}+a^{1}\left(\mu^{1} \Delta \delta u-\nabla \delta \Pi\right)+\delta a\left(\mu^{1} \Delta u^{2}-\nabla \Pi^{2}\right)\right\|_{\widetilde{L}_{t}^{1}\left(\dot{B}_{N \infty}^{-1}\right)} \\
& \lesssim\left\|u^{1}\right\|_{L_{t}^{1}\left(\dot{B}_{N 1}^{2}\right)}\|\delta u\|_{L_{t}^{\infty}\left(\dot{B}_{N \infty}^{-1}\right)}+\left\|a^{1}\right\|_{\widetilde{L}_{t}^{\infty}\left(\dot{B}_{N 1}^{1}\right)}\left(\|\delta u\|_{\widetilde{L}_{t}^{1}\left(\dot{B}_{N \infty}^{1}\right)}+\|\nabla \delta \Pi\|_{\widetilde{L}_{t}^{1}\left(\dot{B}_{N \infty}^{-1}\right)}\right) \\
& +\int_{0}^{t}\|\delta a\|_{\dot{B}_{N \infty}^{0}}\left(\left\|u^{2}\right\|_{\dot{B}_{N 1}^{2}}+\left\|\nabla \Pi^{2}\right\|_{\dot{B}_{N 1}^{0}}\right) d \tau .
\end{aligned}
$$

Par contre pour contrôler $\delta a \operatorname{div}\left[\left(\widetilde{\mu}\left(a^{2}\right)-\mu^{1}\right) \mathcal{M}^{2}\right]$ dans $\widetilde{L}_{t}^{1}\left(\dot{B}_{N \infty}^{-1}\right)$, on n'a pas besoin d'utiliser la proposition 2.5 comme dans le lemme 3.2 grâce à l'hypothèse $a^{i} \in L_{t}^{\infty}\left(\dot{B}_{N 1}^{1}\right)$ au lieu de $L_{t}^{\infty}\left(\dot{B}_{N \infty}^{1} \cap L^{\infty}\right)$. Et par suite les inégalités de Minkowski, (2.6), (2.2), (2.4) et (2.17) impliquent

$$
\begin{aligned}
\| \delta a \operatorname{div}\left[\left(\widetilde{\mu}\left(a^{2}\right)-\mu^{1}\right) \mathcal{M}^{2}\right] & \left\|_{\widetilde{L}_{t}^{1}\left(\dot{B}_{N \infty}^{-1}\right)} \lesssim \int_{0}^{t}\right\| \delta a\left\|_{\dot{B}_{N \infty}^{0}}\right\|\left(\widetilde{\mu}\left(a^{2}\right)-\mu^{1}\right) \mathcal{M}^{2} \|_{\dot{B}_{N 1}^{1}} d \tau \\
& \lesssim \int_{0}^{t}\|\delta a\|_{\dot{B}_{N \infty}^{0}}\left\|\widetilde{\mu}\left(a^{2}\right)-\mu^{1}\right\|_{\dot{B}_{N 1}^{1}}\left\|\nabla u^{2}\right\|_{\dot{B}_{N 1}^{1}} d \tau \\
& \lesssim\left\|a^{2}\right\|_{L_{t}^{\infty}\left(\dot{B}_{N 1}^{1}\right)} \int_{0}^{t}\|\delta a\|_{\dot{B}_{N \infty}^{0}}\left\|u^{2}\right\|_{\dot{B}_{N 1}^{2}} d \tau .
\end{aligned}
$$

D'après les inégalités (2.2), (2.12) et (2.17), on a

$$
\begin{aligned}
\left\|\operatorname{div}\left[\left(\widetilde{\mu}\left(a^{1}\right)-\mu^{1}\right) \delta \mathcal{M}\right]\right\|_{\widetilde{L}_{t}^{1}\left(\dot{B}_{N \infty}^{-1}\right)} & \lesssim\left\|\widetilde{\mu}\left(a^{1}\right)-\mu^{1}\right\|_{\widetilde{L}_{t}^{\infty}\left(\dot{B}_{N 1}^{1}\right)}\|\nabla \delta u\|_{\widetilde{L}_{t}^{1}\left(\dot{B}_{N \infty}^{0}\right)} \\
& \lesssim\left\|a^{1}\right\|_{\widetilde{L}_{t}^{\infty}\left(\dot{B}_{N 1}^{1}\right)}\|\delta u\|_{\widetilde{L}_{t}^{1}\left(\dot{B}_{N \infty}^{1}\right)} .
\end{aligned}
$$

Grâce aux inégalités (2.13), (2.12), (2.18), de Bernstein et de Minkowski, on trouve

$$
\begin{aligned}
\| a^{1} \operatorname{div}\left[\left(\widetilde{\mu}\left(a^{2}\right)\right.\right. & \left.\left.-\widetilde{\mu}\left(a^{1}\right)\right) \mathcal{M}^{2}\right] \|_{\widetilde{L}_{t}^{1}\left(\dot{B}_{N \infty}^{-1}\right)} \\
& \lesssim \int_{0}^{t}\left\|a^{1}\right\|_{\dot{B}_{N 1}^{1}}\left\|\left(\widetilde{\mu}\left(a^{2}\right)-\widetilde{\mu}\left(a^{1}\right)\right) \mathcal{M}^{2}\right\|_{\dot{B}_{N \infty}^{0}} d \tau \\
& \lesssim \int_{0}^{t}\left\|a^{1}\right\|_{\dot{B}_{N 1}^{1}}\left\|\widetilde{\mu}\left(a^{2}\right)-\widetilde{\mu}\left(a^{1}\right)\right\|_{\dot{B}_{N \infty}^{0}}\left\|\nabla u^{2}\right\|_{\dot{B}_{N \infty}^{1} \cap L^{\infty}} d \tau \\
& \lesssim\left\|a^{1}\right\|_{\widetilde{L}_{t}^{\infty}\left(\dot{B}_{N 1}^{1}\right)}\left\|a^{2}\right\|_{L_{t}^{\infty}\left(\dot{B}_{N 1}^{1}\right)} \int_{0}^{t}\|\delta a\|_{\dot{B}_{N \infty}^{0}}\left\|u^{2}\right\|_{\dot{B}_{N 1}^{2}} d \tau .
\end{aligned}
$$

En utilisant les inégalités (2.13), (2.12), (2.17) et Bernstein, on obtient

$$
\begin{aligned}
\left\|a^{1} \operatorname{div}\left[\left(\widetilde{\mu}\left(a^{1}\right)-\mu^{1}\right) \delta \mathcal{M}\right]\right\|_{\widetilde{L}_{t}^{1}\left(\dot{B}_{N \infty}^{-1}\right)} & \lesssim\left\|a^{1}\right\|_{\widetilde{L}_{t}^{\infty}\left(\dot{B}_{N 1}^{1}\right)}\left\|\left(\widetilde{\mu}\left(a^{1}\right)-\mu^{1}\right) \delta \mathcal{M}\right\|_{\widetilde{L}_{t}^{1}\left(\dot{B}_{N \infty}^{0}\right)} \\
& \lesssim\left\|a^{1}\right\|_{\widetilde{L}_{t}^{\infty}\left(\dot{B}_{N 1}^{1}\right)}\|\delta u\|_{\widetilde{L}_{t}^{1}\left(\dot{B}_{N \infty}^{1}\right)} .
\end{aligned}
$$


En suivant la même calcul que pour prouver l'inégalité (3.8), on obtient

$$
\left\|\operatorname{div}\left[\left(\widetilde{\mu}\left(a^{2}\right)-\widetilde{\mu}\left(a^{1}\right)\right) \mathcal{M}^{2}\right]\right\|_{\widetilde{L}_{t}^{1}\left(\dot{B}_{N \infty}^{-1}\right)} \lesssim\left\|a^{2}\right\|_{L_{t}^{\infty}\left(\dot{B}_{N 1}^{1}\right)} \int_{0}^{t}\|\delta a\|_{\dot{B}_{N \infty}^{0}}\left\|u^{2}\right\|_{\dot{B}_{N 1}^{2}} d \tau \text {. }
$$

Donc

$$
\begin{aligned}
& \left.\left\|F\left(a^{i}, u^{i}, \nabla \Pi^{i}\right)\right\|_{\widetilde{L}_{t}^{1}\left(\dot{B}_{N \infty}^{-1}\right)} \lesssim\left\|u^{1}\right\|_{L_{t}^{1}\left(\dot{B}_{N 1}^{2}\right)}\|\delta u\|_{L_{t}^{\infty}\left(\dot{B}_{N \infty}^{-1}\right)}+\left\|a^{1}\right\|_{\widetilde{L}_{t}^{\infty}\left(\dot{B}_{N 1}^{1}\right)}\right) \\
& \quad \times\left(\|\delta u\|_{\widetilde{L}_{t}^{1}\left(\dot{B}_{N \infty}^{1}\right)}+\|\nabla \delta \Pi\|_{\widetilde{L}_{t}^{1}\left(\dot{B}_{N \infty}^{-1}\right)}\right)+\int_{0}^{t}\|\delta a\|_{\dot{B}_{N \infty}^{0}}\left\|u^{2}\right\|_{\dot{B}_{N 1}^{2}} d \tau .
\end{aligned}
$$

Soient

$$
\alpha(\tau) \stackrel{\text { déf }}{=} \sum_{i=1}^{2}\left(\tau\left\|u^{i}\right\|_{L_{\tau}^{\infty}\left(\dot{B}_{N 1}^{0}\right)}+\mu^{1}\left\|u^{i}\right\|_{L_{\tau}^{1}\left(\dot{B}_{N 1}^{2}\right)}\right)
$$

et

$$
W(t) \stackrel{\text { déf }}{=}\|\delta u\|_{L_{t}^{\infty}\left(\dot{B}_{N \infty}^{-1}\right)}+\mu^{1}\|\delta u\|_{\widetilde{L}_{t}^{1}\left(\dot{B}_{N \infty}^{1}\right)}+\|\nabla \delta \Pi\|_{\widetilde{L}_{t}^{1}\left(\dot{B}_{N \infty}^{-1}\right)} .
$$

De même que dans le cas $1 \leq p<N$ et $3 \leq N$, on choisit $T_{1}$ de telle sorte que

$$
e^{C\left\|\nabla u^{2}\right\|_{L_{T_{1}}^{1}\left(\dot{B}_{N 1}^{1}\right)}} \leq 2 \quad \text { et } \quad \mu^{1} \sum_{i=1}^{2}\left\|u^{i}\right\|_{L_{T_{1}}^{1}\left(\dot{B}_{N 1}^{2}\right)}+\left\|\nabla \Pi^{2}\right\|_{L_{T_{1}}^{1}\left(\dot{B}_{N 1}^{0}\right)} \leq c .
$$

Comme $\left\|a^{1}\right\|_{\widetilde{L}_{T^{\star}}^{\infty}\left(\dot{B}_{N \infty}^{1}\right)} \leq c$, les inégalités (3.7), (3.6) et (3.9) impliquent

$$
W(t) \leq 4 C c\left\|a^{2}\right\|_{L_{t}^{\infty}\left(\dot{B}_{N 1}^{1}\right)} \int_{0}^{t} \log \left(e+\frac{\alpha(\tau)}{\left.\|\delta u\|_{\widetilde{L}_{\tau}^{1}\left(\dot{B}_{N \infty}^{1}\right)}^{1}\right)}\right) W(\tau)\left\|u^{2}\right\|_{\dot{B}_{N 1}^{2}} d \tau .
$$

D'où

$$
W(t) \leq C_{t} \int_{0}^{t}\left(1-\log \|\delta u\|_{\widetilde{L}_{\tau}^{1}\left(\dot{B}_{N \infty}^{1}\right)}\right) W(\tau)\left\|u^{2}\right\|_{\dot{B}_{N 1}^{2}} d \tau
$$

dès que $\|\delta u\|_{\widetilde{L}_{t}^{1}\left(\dot{B}_{N \infty}^{1}\right)} \leq 1$ pour $t \in\left[0, T_{1}\right]$. D'autre part, la fonction $x \longmapsto$ $x(1-\log x)$ est croissante sur ]0,1]. Donc

$$
W(t) \leq 4 C_{T_{1}} \int_{0}^{t}(1-\log W(\tau)) W(\tau)\left\|u^{2}\right\|_{\dot{B}_{N 1}^{2}} d \tau .
$$

De nouveau le lemme d'Osgood implique que $W(t)=0$ pour $t \in\left[0, T_{1}\right]$, et par suite l'inégalité (3.7) assure que $a_{1}=a_{2}$. Ensuite par itération, on obtient $(\delta a, \delta u, \nabla \delta \Pi)=0$ sur $[0, T]$.

Remarque 3.1. Pour $N=2$, on commence par le cas $p=2$ (c'est-à-dire $N=p)$ puis par injection, on déduit le résultat pour $1 \leq p \leq 2$. 
Preuve de lemme 3.1. Sous les hypothèses que $p<N$ et $3 \leq N$, les inégalités (2.5) et (2.11) donnent

$$
\begin{aligned}
\| a^{1} \operatorname{div}\left[\left\{\widetilde{\mu}\left(a^{2}\right)\right.\right. & \left.\left.\left.-\widetilde{\mu}\left(a^{1}\right)\right\} \mathcal{M}^{2}\right] \|_{L_{T}^{1}\left(\dot{B}_{p 1}^{\frac{N}{p}}-2\right.}\right) \\
& \lesssim\left\|a^{1}\right\|_{L_{T}^{\infty}\left(\dot{B}_{p}^{\frac{N}{p}} \cap L^{\infty}\right)} \int_{0}^{T}\left\|\left\{\widetilde{\mu}\left(a^{2}\right)-\widetilde{\mu}\left(a^{1}\right)\right\} \mathcal{M}^{2}\right\|_{\dot{B}_{p 1}^{\frac{N}{p}-1}} d t \\
& \lesssim\left\|a^{1}\right\|_{L_{T}^{\infty}\left(\dot{B}_{p \infty}^{\frac{N}{p}} \cap L^{\infty}\right)} \int_{0}^{T}\left\|\widetilde{\mu}\left(a^{2}\right)-\widetilde{\mu}\left(a^{1}\right)\right\|_{\dot{B}_{p 1}^{\frac{N}{p}-1}}\left\|u^{2}\right\|_{\dot{B}_{p 1}^{\frac{N}{p}+1}} d t .
\end{aligned}
$$

Tout d'abord l'inégalité (2.17) implique

$$
\left\|\widetilde{\mu}\left(a^{2}\right)-\widetilde{\mu}\left(a^{1}\right)\right\|_{\dot{B}_{p}^{\frac{N}{p}}} \leq \sum_{i=1}^{2}\left\|\widetilde{\mu}\left(a^{i}\right)\right\|_{\dot{B}_{p \infty}^{\frac{N}{p}}} \lesssim \sum_{i=1}^{2}\left\|a^{i}\right\|_{\dot{B}_{p}^{p}} .
$$

Comme $N>p$ et $N \geq 3$, alors les inégalités (2.18), (2.5), (3.3) et l'inégalité de Hölder assurent que pour tout $t \leq T$

$$
\begin{aligned}
& \left\|\widetilde{\mu}\left(a^{2}\right)-\widetilde{\mu}\left(a^{1}\right)\right\|_{\dot{B}_{p}^{\frac{N}{p}-2}} \lesssim\|\delta a\|_{\dot{B}_{p \infty}^{\frac{N}{p}-2}} \sum_{i=1}^{2}\left\|a^{i}\right\|_{\dot{B}_{p}^{\frac{N}{p}} \cap L^{\infty}} \\
& \lesssim \exp \left(C\left\|\nabla u^{2}\right\|_{L_{t}^{1}\left(\dot{B}_{p 1}^{\frac{N}{p}}\right)}\right)\|\delta u\|_{L_{t}^{1}\left(\dot{B}_{p \infty}^{\frac{N}{p}-1}\right)}\left\|a^{1}\right\|_{L_{t}^{\infty}\left(\dot{B}_{p \infty}^{\frac{N}{p}}\right)} \sum_{i=1}^{2}\left\|a^{i}\right\|_{\dot{B}_{p \infty}^{\frac{N}{p}} \cap L^{\infty}} \\
& \lesssim t \exp \left(C\left\|\nabla u^{2}\right\|_{L_{t}^{1}\left(\dot{B_{p}} \frac{N}{p}\right)}\right) \sum_{i=1}^{2}\left\|u^{i}\right\|_{L_{t}^{\infty}\left(\dot{B}_{p \infty}^{\frac{N}{p}}-1\right)}\left\|a^{1}\right\|_{L_{t}^{\infty}\left(\dot{B}_{p}^{\frac{N}{p}}\right)} \sum_{i=1}^{2}\left\|a^{i}\right\|_{\dot{B}_{p}^{\frac{N}{p}} \cap L^{\infty}} \\
& \lesssim t \exp \left(C\left\|\nabla u^{2}\right\|_{L_{t}^{1}\left(\dot{B_{p}}\right)}\right) \sum_{i=1}^{2}\left\|u^{i}\right\|_{L_{t}^{\infty}\left(\dot{B}_{p \infty}^{\frac{N}{p}}-1\right)} \sum_{i=1}^{2}\left\|a^{i}\right\|_{\dot{B}_{p \infty}^{\frac{N}{p}} \cap L^{\infty}}
\end{aligned}
$$

et par suite pour tout $t \leq T$, on a

$$
\left\|\widetilde{\mu}\left(a^{2}\right)-\widetilde{\mu}\left(a^{1}\right)\right\|_{\dot{B}_{p \infty}^{\frac{N}{p}-2}} \leq C_{T} \quad \text { avec } C_{T} \underset{T \rightarrow 0}{\longrightarrow} 0 .
$$

D'après les inégalités (2.18), (2.5) et (2.17), on a

$$
\left\|\widetilde{\mu}\left(a^{2}\right)-\widetilde{\mu}\left(a^{1}\right)\right\|_{\dot{B}_{p \infty}^{\frac{N}{p}-1}} \lesssim\|\delta a\|_{\dot{B}_{p \infty}^{\frac{N}{p}-1}}\left\|a^{i}\right\|_{\dot{B}_{p \infty}^{\frac{N}{p}} \cap L^{\infty}} \leq C_{T}^{\prime}\|\delta a\|_{\dot{B}_{p \infty}^{\frac{N}{p}-1}} .
$$

En appliquant l'inégalité (2.19), le fait que

$$
\log \left(e+\alpha x^{-1}\right) \leq(1-\log x) \log (e+\alpha), \quad \forall \alpha \geq 0 \text { et } x \in(0,1]
$$

et la croissance de la fonction $x \longmapsto x(1-\log x)$ sur $(0,1]$, on obtient

$$
\left\|\widetilde{\mu}\left(a^{2}\right)-\widetilde{\mu}\left(a^{1}\right)\right\|_{\dot{B}_{p 1}^{\frac{N}{p}-1}} \lesssim\|\delta a\|_{\dot{B}_{p \infty}^{\frac{N}{p}-1}}\left\{1-\log \left(\|\delta a\|_{\dot{B}_{p \infty}^{\frac{N}{p}-1}}\right)\right\},
$$


car on a $\delta a \in C\left([0, T] ; \dot{B}_{p \infty}^{\frac{N}{p}-1}\right)$ et $\delta a(0)=0$, donc on peut choisir $T_{2}$ de telle sorte que $C_{T}^{\prime}\|\delta a(t)\|_{\dot{B}_{p}^{\frac{N}{p}-1}} \leq 1$ pour tout $t \in[0, T]$. Donc

$$
\left\|\widetilde{\mu}\left(a^{2}\right)-\widetilde{\mu}\left(a^{1}\right)\right\|_{\dot{B}_{p 1}^{\frac{N}{p}-1}} \lesssim\|\delta a\|_{\dot{B}_{p \infty}^{\frac{N}{p}-1}}\left\{1-\log \left(\|\delta a\|_{\dot{B}_{p \infty}^{\frac{N}{p}-1}}\right)\right\} \forall t \in\left[0, T_{2}\right] .
$$

De même, on peut supposer $C_{T_{2}}\|\delta a\|_{\dot{B}_{p \infty}^{\frac{N}{p}-1}} \leq \sqrt{\|\delta a\|_{\dot{B}_{p}^{p}}^{\frac{N}{p}-1}}$, on conclut que

$$
\begin{aligned}
\| a^{1} \operatorname{div}[ & \left.\left\{\widetilde{\mu}\left(a^{2}\right)-\widetilde{\mu}\left(a^{1}\right)\right\} \mathcal{M}^{2}\right] \|_{L_{T}^{1}\left(\dot{B}_{p 1}^{\frac{N}{p}-2}\right)} \\
& \lesssim\left\|a^{1}\right\|_{L_{T}^{\infty}\left(\dot{B}_{p}^{\frac{N}{p}} \cap L^{\infty}\right)} \int_{0}^{T}\|\delta a\|_{\dot{B}_{p \infty}^{\frac{N}{p}-1}}\left(1-\log \|\delta a\|_{\dot{B}_{p \infty}^{\frac{N}{p}-1}}\right)\left\|u^{2}\right\|_{\dot{B}_{p 1}^{\frac{N}{p}+1}} d t .
\end{aligned}
$$

Preuve de la proposition 3.2. On applique l'opérateur $\dot{\Delta}_{q}$ au système $(\mathcal{T})$. On obtient :

$\partial_{t} \dot{\Delta}_{q} f+v \cdot \nabla \dot{\Delta}_{q} f=\dot{\Delta}_{q} F+\left[v, \dot{\Delta}_{q}\right] \cdot \nabla f$ où $\left[v, \dot{\Delta}_{q}\right] \cdot \nabla f=v \cdot \nabla \dot{\Delta}_{q} f-\dot{\Delta}_{q}(v \cdot \nabla f)$.

Dans le cas où $1<p<\infty$, on multiplie l'égalité précédente par

$$
\left|\dot{\Delta}_{q} f\right|^{p-1} \operatorname{sign}\left(\dot{\Delta}_{q} f\right)
$$

et on obtient du fait que $\operatorname{div} v=0$ et grâce à l'inégalité de Hölder :

$$
\frac{1}{p} \frac{d}{d t}\left\|\dot{\Delta}_{q} f\right\|_{L^{p}}^{p} \leq\left\|\dot{\Delta}_{q} f\right\|_{L^{p}}^{p-1}\left(\left\|\left[v, \dot{\Delta}_{q}\right] \cdot \nabla f\right\|_{L^{p}}+\left\|\dot{\Delta}_{q} F\right\|_{L^{p}}\right) .
$$

On en déduit que,

$$
2^{q s}\left\|\dot{\Delta}_{q} f\right\|_{L_{T}^{\infty}\left(L^{p}\right)} \leq 2^{q s}\left\|\dot{\Delta}_{q} f_{0}\right\|_{L^{p}}+2^{q s}\left\|\dot{\Delta}_{q} F\right\|_{L_{T}^{1}\left(L^{p}\right)}+2^{q s}\left\|\left[v, \dot{\Delta}_{q}\right] \cdot \nabla f\right\|_{L_{T}^{1}\left(L^{p}\right)} .
$$

Donc grâce au lemme A.1 [8] et l'inégalité de Minkowski, on obtient

$$
\|f\|_{\widetilde{L}_{T}^{\infty}\left(\dot{B}_{p r}^{s}\right)} \leq\left\|f_{0}\right\|_{\dot{B}_{p r}^{s}}+\int_{0}^{T}\|F\|_{\dot{B}_{p r}^{s}} d t+C \int_{0}^{T}\|\nabla v\|_{\dot{B}_{p r}^{p} \cap L^{\infty}}\|f\|_{\dot{B}_{p r}^{s}} d t .
$$

On en déduit alors la majoration (3.3) à partir d'un argument du type lemme de Gronwall. Les cas $p=1$ et $p=\infty$ s'obtiennent par passage à la limite. La continuité en temps est prouvée dans [8].

Preuve de la proposition 3.3. Rappelons tout d'abord une inégalité semblable à l'inégalité de Bernstein (voir [10]) qui nous permet de gagner deux dérivées à partir du laplacien dans $L^{p}$ si $1<p<\infty$. 
Lemme 3.3. Soient $N \geq 1,1<p<+\infty$ et $u \in L^{p}\left(\mathbb{R}^{N}\right)$ tel que supp $\mathcal{F} u \subset C\left(0, R_{1}, R_{2}\right)$ (avec $\left.0<R_{1}<R_{2}\right)$. Alors il existe une constante $K$ strictement positive dépendant de $N$ et $\frac{R_{1}}{R_{2}}$ telle que

$$
K \frac{R_{1}^{2}}{p^{2}} \int_{\mathbb{R}^{N}}|u|^{p} d x \leq \int_{\mathbb{R}^{N}}|\nabla u|^{2}|u|^{p-2} d x=-\frac{1}{p-1} \int_{\mathbb{R}^{N}} \Delta u|u|^{p-2} u d x .
$$

En suivant la même démarche que dans la démontration de la proposition 3.2 , on trouve après l'application de $\mathcal{P}$ et de l'inégalité (3.11) que

$$
\begin{aligned}
2^{q s}\left\|\dot{\Delta}_{q} u\right\|_{L_{T}^{\infty}\left(L^{p}\right)}+k \nu 2^{q(s+2)}\left\|\dot{\Delta}_{q} u\right\|_{L_{T}^{1}\left(L^{p}\right)} & \leq 2^{q s}\left\|\dot{\Delta}_{q} u_{0}\right\|_{L^{p}}+2^{q s}\left\|\dot{\Delta}_{q} \mathcal{P} g\right\|_{L_{T}^{1}\left(L^{p}\right)} \\
& +2^{q s}\left\|\left[v, \dot{\Delta}_{q}\right] \cdot \nabla u\right\|_{L_{T}^{1}\left(L^{p}\right)}
\end{aligned}
$$

De nouveau on déduit grâce au lemme A.1 [8] et l'inégalité de Minkowski que

$$
\begin{aligned}
\|u\|_{\widetilde{L}_{T}^{\infty}\left(\dot{B}_{p r}^{s}\right)}+k \nu\|u\|_{\widetilde{L}_{T}^{1}\left(\dot{B}_{p r}^{s+2}\right)} & \leq\left\|u_{0}\right\|_{\dot{B}_{p r}^{s}}+\|\mathcal{P} g\|_{\widetilde{L}_{T}^{1}\left(\dot{B}_{p r}^{s}\right)} \\
& +C \int_{0}^{T}\|\nabla v(t)\|_{\dot{B}_{p r}^{p} L^{\infty}}\|u(t)\|_{\dot{B}_{p r}^{s}} d t .
\end{aligned}
$$

Pour estimer $\nabla \Pi$ dans $\widetilde{L}_{T}^{1}\left(\dot{B}_{p r}^{s}\right)$, on applique l'opérateur de divergence, on trouve l'équation suivante

$$
\Delta \Pi=\operatorname{div} \mathcal{Q} g-\operatorname{div}(v \cdot \nabla u) .
$$

Donc l'inégalité (2.2), l'inégalité de Minkowski et le fait que div $v=\operatorname{div} u=0$, impliquent

$$
\begin{aligned}
\|\Delta \Pi\|_{\widetilde{L}_{T}^{1}\left(\dot{B}_{p r}^{s-1}\right)} & \leq c\|\mathcal{Q} g\|_{\widetilde{L}_{T}^{1}\left(\dot{B}_{p r}^{s}\right)}+\left\|\sum_{1 \leq i, j \leq N} \partial_{j}\left(\partial_{i} v^{j} u^{i}\right)\right\|_{L_{T}^{1}\left(\dot{B}_{p r}^{s-1}\right)} \\
& \lesssim\|\mathcal{Q} g\|_{\widetilde{L}_{T}^{1}\left(\dot{B}_{p r}^{s}\right)}+\|u \cdot \nabla v\|_{L_{T}^{1}\left(\dot{B}_{p r}^{s}\right)}
\end{aligned}
$$

Et par suite les inégalités (2.5) et l'inégalité de Minkowski entrainent

$$
\|u \cdot \nabla v\|_{L_{T}^{1}\left(\dot{B}_{p r}^{s}\right)} \lesssim \int_{0}^{T}\|\nabla v\|_{\dot{B}_{p \infty}^{\frac{N}{p}} \cap L^{\infty}}\|u\|_{\dot{B}_{p r}^{s}} d t .
$$

Puis en reportant cette expression dans l'inégalité précédente, on obtient grâce à l'inégalité (2.2) que

$$
\|\nabla \Pi\|_{\widetilde{L}_{T}^{1}\left(\dot{B}_{p r}^{s}\right)} \lesssim\|\mathcal{Q} g\|_{\widetilde{L}_{T}^{1}\left(\dot{B}_{p r}^{s}\right)}+\int_{0}^{T}\|\nabla v\|_{\dot{B}_{p \infty}^{p} \cap L^{\infty}}\|u\|_{\dot{B}_{p r}^{s}} d t
$$


Pour conclure il suffit d'utiliser les inégalités (3.12), (3.13) et un argument du type lemme de Gronwall. Pour montrer l'inégalité (3.5) on reprend les calculs qui conduisent à l'inégalité (3.4). Puisque le lemme A.1 [8] est vrai pour $p \geq 2$ et $s=-\frac{N}{p}$ car le seul terme que posé des problemes est $R\left(v^{j}, \partial_{j} u\right)$. Mais par définition de reste et le fait que $p \geq 2$, on touve à partir des inégalités de Bernstein et de Hölder que

$\left\|\partial_{j} R\left(v^{j}, u\right)\right\|_{L_{T}^{1}\left(B_{p}^{-\frac{N}{p}}\right)} \lesssim\left\|R\left(v^{j}, u\right)\right\|_{L_{T}^{1}\left(B_{p}^{1-\frac{N}{p}}\right)} \lesssim \int_{0}^{T}\|u(\tau)\|_{B_{p}^{-\frac{N}{p}}}\|v(\tau)\|_{B_{p 1}^{\frac{N}{p}+1}} d \tau$.

Pour l'estimation de la pression, on utilise l'inégalité (2.13) que implique que

$$
\|u \cdot \nabla v\|_{L_{T}^{1}\left(\dot{B}_{p}^{\left.-\frac{N}{p}\right)}\right.} \lesssim \int_{0}^{T}\|u(\tau)\|_{\dot{B}_{p}^{-\frac{N}{p}}}\|\nabla v(\tau)\|_{\dot{B}_{p 1}^{\frac{N}{p}}} d \tau \text {. }
$$

D'où la proposition.

\section{Existence}

L'objectif de cette section est de prouver le théorème 1.2 dont la démonstration répose sur une méthode itérative. On prouve ensuite l'existence dans l'espace de Besov qui s'appuie sur le théorème 1.2 et le fait que $H^{s_{0}}\left(\mathbb{R}^{N}\right) \hookrightarrow$ $\dot{B}_{q 1}^{s}\left(\mathbb{R}^{N}\right)$ dès que $s_{0}>s+\frac{N}{2}-\frac{N}{q}, s>\frac{N}{q}-\frac{N}{2}$ et $q \geq 2$.

\subsection{Démonstration du théorème 1.2}

Le but de cette section est d'étudier l'existence locale des solutions régulières du système ( $\widetilde{\mathrm{INS}}$ ). Rappelons tout d'abord l'estimation d'énergie dans $L^{2}$ :

Lemme 4.1. Soit $(\rho, u, \nabla \Pi)$ une solution sur $[0, T]$ du système linéaire suivant

$$
\left\{\begin{array}{l}
\partial_{t} \rho+v \cdot \nabla \rho=\rho h \\
\rho\left(\partial_{t} u+v \cdot \nabla u\right)+\nabla \Pi-\operatorname{div}(\mu(\rho) \mathcal{M})=\rho f \\
\operatorname{div} u=0 \\
(\rho, u)_{\mid t=0}=\left(\rho_{0}, u_{0}\right)
\end{array}\right.
$$

avec $0<\mu \leq \mu$ et $v$ un champ de vecteurs régulier à divergence nulle. Alors pour tout $\bar{t} \in[0, T]$ on a

$$
\begin{aligned}
& \|\rho(t)\|_{L^{p}} \leq\left\|\rho_{0}\right\|_{L^{p}}+\int_{0}^{t}\|(\rho h)(s)\|_{L^{p}} d s \quad \forall p \in[1, \infty], \\
& e^{-\frac{1}{2} \int_{0}^{t}\|h(\tau)\|_{L^{\infty}} d \tau}\|(\sqrt{\rho} u)(t)\|_{L^{2}} \leq\left\|\sqrt{\rho_{0}} u_{0}\right\|_{L^{2}} \\
& +\int_{0}^{t} e^{-\frac{1}{2} \int_{0}^{\tau}\|h(s)\|_{L^{\infty}} d s}\|(\sqrt{\rho} f)(\tau)\|_{L^{2}} d \tau .
\end{aligned}
$$


Preuve. La démonstration du lemme est la même que celle de la proposition 2.1 de [9], puisque

$$
(-\operatorname{div}(\mu(\rho) \mathcal{M}) \mid u)=\int_{\mathbb{R}^{N}} \mu(\rho) \mathcal{M} \nabla u d x \geq \frac{1}{2} \underline{\mu}\|\mathcal{M}\|_{L^{2}}^{2}
$$

et par intégration par parties, on a

$$
\|\mathcal{M}\|_{L^{2}}=\sqrt{2}\|\nabla u\|_{L^{2}}
$$

Avant d'entamer l'étude du système ( $\widetilde{\mathrm{INS}})$, il est utile de donner une estimation des solutions du système de Stokes à densité et viscosité variables $(\mathrm{M})$ :

$$
\left\{\begin{array}{l}
\partial_{t} u+v \cdot \nabla u+(1+a)(\nabla \Pi-\operatorname{div}\{\widetilde{\mu}(a) \mathcal{M}\})=g \\
\operatorname{div} u=0 \\
u_{\mid t=0}=u_{0}
\end{array}\right.
$$

avec $a, g, v$ et $u_{0}$ données. On suppose que $\underline{a} \stackrel{\text { déf }}{=} \inf _{x}(1+a)>0, \mu$ vérifie (1.1).

Plus précisément, on a la proposition suivante qui généralise un résultat dû à R. Danchin (voir [9, proposition 3.3]).

Proposition 4.1. Soient $m \in[1, \infty]$,

$$
s \in\left(2-\frac{2}{m}, \alpha+\frac{N}{2}+\inf \left(0,1+\frac{1}{2} \alpha \vee 1-\frac{2}{m}\right)\right]
$$

tel que $\frac{s}{2}+\frac{1}{m} \leq \frac{N}{2}+\alpha$ et $\alpha>0$. On suppose que $a_{0} \in H^{\frac{N}{2}+\alpha}\left(\mathbb{R}^{N}\right)$ vérifie $\underline{a} \stackrel{\text { déf }}{=} \inf \left(1+a_{0}\right)>0$, que $u_{0}$ est un champ de vecteurs à divergence nulle avec coefficients dans $H^{s}\left(\mathbb{R}^{N}\right)$ et $g$ un champ de vecteurs à coefficients dans $\widetilde{L}_{T}^{1}\left(H^{s}\left(\mathbb{R}^{N}\right)\right)$. Supposons que $a \in \widetilde{C}_{T}\left(H^{\frac{N}{2}+\alpha}\left(\mathbb{R}^{N}\right)\right.$ ) (avec de plus $\nabla$ a à coefficients dans $L_{T}^{\infty}\left(L^{\infty}\left(\mathbb{R}^{N}\right)\right)$ si $\left.\alpha=1\right)$. Soit $v$ un champ de vecteurs à divergence nulle tel que $\nabla v$ soit à coefficients dans $L^{1}\left(0, T ; B_{2}^{\frac{N}{2}}\left(\mathbb{R}^{N}\right) \cap L^{\infty}\left(\mathbb{R}^{N}\right)\right)$ si $s<\frac{N}{2}+1$ et dans $L^{1}\left(0, T ; H^{s}\left(\mathbb{R}^{N}\right)\right)$ si $s>\frac{N}{2}+1$, avec $s \neq 1+\frac{N}{2}$ si $\nabla v \neq 0$. Soit $u \in\left(\widetilde{L}_{T}^{\infty}\left(H^{s}\left(\mathbb{R}^{N}\right)\right)\right)^{N}$ une solution du système $(M)$ sur $[0, T] \times \mathbb{R}^{N}$ et

$$
\nabla \Pi \in\left(\widetilde{L}_{T}^{1}\left(H^{s}\left(\mathbb{R}^{N}\right)\right)\right)^{N}+\left(\widetilde{L}_{T}^{m}\left(H^{s-2+\frac{2}{m}}\left(\mathbb{R}^{N}\right)\right)\right)^{N} .
$$

On note $\bar{\mu} \stackrel{\text { déf }}{=} \underline{a} \underline{\mu}$. Alors il existe une constante strictement positive $C=$ $C(s, N, \alpha, m, \mu)$ telle que

$$
\begin{array}{r}
\|u\|_{\widetilde{L}_{T}^{\infty}\left(H^{s}\right)}+\bar{\mu}^{\frac{1}{m}}\|u\|_{\widetilde{L}_{T}^{m}\left(H^{\left.s+\frac{2}{m}\right)}\right.} \leq C \exp \left(C A_{T}^{\frac{s+2}{\alpha^{\prime}}} V(T)\right)\left(\left\|u_{0}\right\|_{H^{s}}+A_{T}^{\frac{s+2}{\alpha^{\prime}}} \times\right. \\
\left.\times\left\{\|g\|_{\widetilde{L}_{T}^{1}\left(H^{s}\right)}+\bar{\mu}^{\frac{1}{m}} A_{T}^{\frac{2}{m \alpha^{\prime}}+\left[\frac{N}{2}+\alpha\right]}\|u\|_{\widetilde{L}_{T}^{m}\left(H^{s+\frac{2}{m}-\alpha^{\prime}}\right)}\right\}\right),
\end{array}
$$


avec

$$
\begin{gathered}
V(t) \stackrel{\text { déf }}{=} \begin{cases}\int_{0}^{t}\|\nabla v\|_{B_{2 \infty}^{\frac{N}{2}} \cap L^{\infty}} d \tau & \text { si } s<\frac{N}{2}+1 \\
\int_{0}^{t}\|\nabla v\|_{H^{s-1}} d \tau & \text { si } s>\frac{N}{2}+1,\end{cases} \\
A_{T} \stackrel{\text { déf }}{=} \begin{cases}1+(1+\underline{a})\|a\|_{\widetilde{L}_{T}^{\infty}\left(H^{\frac{N}{2}+\alpha}\right)} & \text { si } \alpha \neq 1 \\
1+(1+\underline{a})\left(\|a\|_{\widetilde{L}_{T}^{\infty}\left(H^{\frac{N}{2}+1}\right)}+\|\nabla a\|_{L_{T}^{\infty}\left(L^{\infty}\right)}\right) & \text { si } \alpha=1\end{cases}
\end{gathered}
$$

et $\alpha^{\prime}$ est un réel strictement positif vérifiant $\alpha^{\prime} \leq \inf \left(1, \frac{1}{2} \alpha, \frac{s-2+\frac{2}{m}}{2}\right)$.

De plus, on a $\nabla \Pi=\nabla \Pi^{1}+\nabla \Pi^{2}$ avec

$$
\underline{a}\left\|\nabla \Pi^{1}\right\|_{\widetilde{L}_{T}^{1}\left(H^{s}\right)} \lesssim A_{T}^{\frac{s+1}{\alpha^{\prime}}}\left(\|\mathcal{Q} g\|_{\widetilde{L}_{T}^{1}\left(H^{s}\right)}+\int_{0}^{T} V(t)\|u(t)\|_{H^{s}} d t\right)
$$

et

$$
\begin{aligned}
\underline{a}\left\|\nabla \Pi_{2}\right\|_{\widetilde{L}_{T}^{m}\left(H^{s+\frac{2}{m}-2-\alpha^{\prime}}\right)} \lesssim & A_{T}^{\frac{s}{\alpha^{\prime}}}\|a\|_{\widetilde{L}_{T}^{\infty}\left(H^{\frac{N}{2}+\alpha}\right)}\|u\|_{\widetilde{L}_{T}^{m}\left(H^{s+\frac{2}{m}-\alpha^{\prime}}\right)} \\
& \times\left\{\mu^{1}+\|a\|_{\widetilde{L}_{T}^{\infty}\left(H^{\frac{N}{2}+\alpha}\right)}\right\} .
\end{aligned}
$$

Si $v=u$, alors les estimations restent vraies avec $V(t)=\int_{0}^{t}\|\nabla u\|_{L^{\infty}} d \tau$ (même si $\left.s=\frac{N}{2}+1\right)$.

Preuve. Lorsque $\mu$ est une constante strictement positive, R. Danchin a démontré dans [9] proposition 3.3 le même résultat, on va donc suivre la même démarche.

On applique l'opérateur $\Delta_{q}$ à $(M)$, on obtient pour $q \geq-1$

$$
\begin{aligned}
\partial_{t} \Delta_{q} u+v \cdot \nabla \Delta_{q} u & +\Delta_{q} \nabla \Pi-\operatorname{div}\left((1+a) \widetilde{\mu}(a) \Delta_{q} \mathcal{M}\right) \\
& =\Delta_{q} g+\left[v, \Delta_{q}\right] \cdot \nabla u-\Delta_{q}(a \nabla \Pi)+R_{q}
\end{aligned}
$$

avec

$$
\begin{aligned}
R_{q} & =\Delta_{q}[(1+a) \operatorname{div}\{\widetilde{\mu}(a) \mathcal{M}\}]-\operatorname{div}\left((1+a) \widetilde{\mu}(a) \Delta_{q} \mathcal{M}\right) \\
& =\Delta_{q}\left[(1+a) \operatorname{div}\left\{\left(\widetilde{\mu}(a)-\mu^{1}\right) \mathcal{M}\right\}\right]-\operatorname{div}\left[(1+a)\left\{\widetilde{\mu}(a)-\mu^{1}\right\} \Delta_{q} \mathcal{M}\right] \\
& +\mu^{1} \Delta_{q}(a \operatorname{div} \mathcal{M})-\mu^{1} \operatorname{div}\left(a \Delta_{q} \mathcal{M}\right) \\
& =\Delta_{q}\left[a \operatorname{div}\left\{\left(\widetilde{\mu}(a)-\mu^{1}\right) \mathcal{M}\right\}\right]-\operatorname{div}\left[a \Delta_{q}\left(\left\{\widetilde{\mu}(a)-\mu^{1}\right\} \mathcal{M}\right)\right] \\
& +\mu^{1} \Delta_{q}(a \operatorname{div} \mathcal{M})-\mu^{1} \operatorname{div}\left(a \Delta_{q} \mathcal{M}\right)-\operatorname{div}\left\{(1+a)\left[\widetilde{\mu}(a)-\mu^{1}, \Delta_{q}\right] \cdot \mathcal{M}\right\} \\
& =R_{q}^{1}+\operatorname{div} R_{q}^{2}
\end{aligned}
$$

où $R_{q}^{2}=-(1+a)\left[\widetilde{\mu}(a)-\mu^{1}, \Delta_{q}\right] \cdot \mathcal{M}$ et $R_{q}^{1}=R_{q}-\operatorname{div} R_{q}^{2}$. 
Par intégration par parties et en utilisant l'inégalité de Bernstein, on trouve

$$
\left|\left(\operatorname{div} R_{q}^{2} \mid \Delta_{q} u\right)\right| \lesssim 2^{q}\left\|R_{q}^{2}\right\|_{L^{2}}\left\|\Delta_{q} u\right\|_{L^{2}}
$$

Comme div $u=0$, alors grâce à l'inégalité de Bernstein on trouve avec $\bar{\mu} \stackrel{\text { déf }}{=} \underline{a} \underline{\mu}$,

$$
\begin{aligned}
\left(-\operatorname{div}\left((1+a) \widetilde{\mu}(a) \Delta_{q} \mathcal{M} \mid \Delta_{q} u\right)\right. & =\int_{\mathbb{R}^{N}}(1+a) \widetilde{\mu}(a) \Delta_{q} \mathcal{M} \Delta_{q} \nabla u d x \\
& =\frac{1}{2} \int_{\mathbb{R}^{N}}(1+a) \widetilde{\mu}(a) \Delta_{q} \mathcal{M} \Delta_{q} \mathcal{M} d x \\
& \geq \frac{1}{2} \bar{\mu}\left\|\Delta_{q} \mathcal{M}\right\|_{L^{2}}^{2} \\
& \geq \bar{\mu}\left\|\Delta_{q} \nabla u\right\|_{L^{2}}^{2}
\end{aligned}
$$

puisque par intégration par parties, on obtient

$$
\left\|\Delta_{q} \mathcal{M}\right\|_{L^{2}}^{2}=2\left\|\Delta_{q} \nabla u\right\|_{L^{2}}^{2}+2 \int_{\mathbb{R}^{N}} \partial_{j} \Delta_{q} u^{i} \partial_{i} \Delta_{q} u^{j}=2\left\|\Delta_{q} \nabla u\right\|_{L^{2}}^{2} .
$$

En prenant le produit scalaire au sens $L^{2}$ de l'égalité (4.1) avec $\Delta_{q} u$, on obtient

$$
\begin{aligned}
& \frac{1}{2} \frac{d}{d t}\left\|\Delta_{q} u\right\|_{L^{2}}^{2}+\bar{\mu}\left\|\Delta_{q} \nabla u\right\|_{L^{2}}^{2} \leq\left\|\Delta_{q} u\right\|_{L^{2}}\left(\left\|R_{q}^{1}\right\|_{L^{2}}+2^{q}\left\|R_{q}^{2}\right\|_{L^{2}}\right. \\
& \left.\quad+\left\|\left[v, \Delta_{q}\right] \cdot \nabla u\right\|_{L^{2}}+\left\|\Delta_{q} T_{\nabla a} \Pi\right\|_{L^{2}}+\left\|\Delta_{q} T_{\nabla \Pi}^{\prime} a\right\|_{L^{2}}+\left\|\Delta_{q} \mathcal{P} g\right\|_{L^{2}}\right) .
\end{aligned}
$$

Pour $q \geq 0, \mathcal{F}\left(\Delta_{q} u\right)$ est supportée dans $2^{q} C$, donc il existe une constante strictement positive $c_{1}$ telle que :

$$
\left\|\nabla \Delta_{q} u\right\|_{L^{2}}^{2} \geq c_{1} 2^{2 q}\left\|\Delta_{q} u\right\|_{L^{2}}^{2}
$$

d'où

$$
\begin{aligned}
& \frac{1}{2} \frac{d}{d t}\left\|\Delta_{q} u\right\|_{L^{2}}^{2}+c_{1} 2^{2 q} \bar{\mu}\left\|\Delta_{q} u\right\|_{L^{2}}^{2} \leq\left\|\Delta_{q} u\right\|_{L^{2}}\left(\left\|R_{q}^{1}\right\|_{L^{2}}+2^{q}\left\|R_{q}^{2}\right\|_{L^{2}}\right. \\
& \left.\quad+\left\|\left[v, \Delta_{q}\right] \cdot \nabla u\right\|_{L^{2}}+\left\|\Delta_{q} T_{\nabla a} \Pi\right\|_{L^{2}}+\left\|\Delta_{q} T_{\nabla \Pi}^{\prime} a\right\|_{L^{2}}+\left\|\Delta_{q} \mathcal{P} g\right\|_{L^{2}}\right) .
\end{aligned}
$$

Soit $\varepsilon>0$, comme

$$
\frac{1}{2} \frac{d}{d t}\left\|\Delta_{q} u\right\|_{L^{2}}^{2}=\left(\left\|\Delta_{q} u\right\|_{L^{2}}^{2}+\varepsilon\right)^{\frac{1}{2}} \frac{d}{d t}\left(\left\|\Delta_{q} u\right\|_{L^{2}}^{2}+\varepsilon\right)^{\frac{1}{2}}
$$


alors

$$
\begin{aligned}
e^{-k \bar{\mu} 2^{2 q} t} \frac{d}{d t} & {\left[e^{k \bar{\mu} 2^{2 q} t}\left(\left\|\Delta_{q} u\right\|_{L^{2}}^{2}+\varepsilon\right)^{\frac{1}{2}}\right] \leq\left\|R_{q}^{1}\right\|_{L^{2}}+2^{q}\left\|R_{q}^{2}\right\|_{L^{2}} } \\
& +\left\|\Delta_{q} T_{\nabla a} \Pi\right\|_{L^{2}}+\left\|\Delta_{q} T_{\nabla \Pi}^{\prime} a\right\|_{L^{2}}+\left\|\left[v, \Delta_{q}\right] \cdot \nabla u\right\|_{L^{2}} \\
& +\left\|\Delta_{q} \mathcal{P} g\right\|_{L^{2}}+c_{1} 2^{2 q} \widetilde{\mu} \varepsilon^{\frac{1}{2}}
\end{aligned}
$$

avec

$$
k \stackrel{\text { déf }}{=} \begin{cases}0 & \text { si } q=-1 \\ c_{1} & \text { sinon. }\end{cases}
$$

On décompose $\Pi$ sous la forme suivante $\Pi=\Pi_{1}+\Pi_{2}$ avec

$$
\begin{array}{lll}
\operatorname{div}\left((1+a) \nabla \Pi_{1}\right)=\operatorname{div} G & \text { où } \quad G \stackrel{\text { déf }}{=} g-T_{\nabla u} v-T_{\nabla v}^{\prime} u, \\
\operatorname{div}\left((1+a) \nabla \Pi_{2}\right)=\operatorname{div} H & \text { où } \quad H \stackrel{\text { déf }}{=}(1+a) \operatorname{div}(\widetilde{\mu}(a) \mathcal{M}) .
\end{array}
$$

En intégrant (4.2) par rapport au temps, puis en faisant tendre $\varepsilon$ vers 0 puis en multipliant par $2^{q s}$ et en prenant la norme $l^{2}$, on trouve

$$
\begin{aligned}
& \|u\|_{\widetilde{L}_{T}^{\infty}\left(H^{s}\right)}+\bar{\mu}^{\frac{1}{m}}\|u\|_{\widetilde{L}_{T}^{m}\left(H^{s+\frac{2}{m}}\right)} \lesssim\left\|u_{0}\right\|_{H^{s}}+\bar{\mu}^{\frac{1}{m}}\left\|\Delta_{-1} u\right\|_{L_{T}^{m}\left(L^{2}\right)}+\|\mathcal{P} g\|_{\widetilde{L}_{T}^{1}\left(H^{s}\right)} \\
& +\left\|T_{\nabla a} \Pi_{1}\right\|_{\widetilde{L}_{T}^{1}\left(H^{s}\right)}+\bar{\mu}^{\frac{1}{m}-1}\left\|T_{\nabla a} \Pi_{2}\right\|_{\widetilde{L}_{T}^{m}\left(H^{s+\frac{2}{m}-2}\right)}+\left\|T_{\nabla \Pi_{1}}^{\prime} a\right\|_{\widetilde{L}_{T}^{1}\left(H^{s}\right)} \\
& +\bar{\mu}^{\frac{1}{m}-1}\left\|T_{\nabla \Pi_{2}}^{\prime} a\right\|_{\widetilde{L}_{T}^{m}\left(H^{s+\frac{2}{m}-2}\right)}+\left(\sum_{q \geq-1} 2^{2 q s}\left\|\left[v, \Delta_{q}\right] \cdot \nabla u\right\|_{L_{T}^{1}\left(L^{2}\right)}\right)^{\frac{1}{2}} \\
& +\bar{\mu}^{\frac{1}{m}-1}\left\{\left(\sum_{q \geq-1} 2^{2 q\left(s+\frac{2}{m}-2\right)}\left\|R_{q}^{1}\right\|_{L_{T}^{m}\left(L^{2}\right)}^{2}\right)^{\frac{1}{2}}+\left(\sum_{q \geq-1} 2^{2 q\left(s+\frac{2}{m}-1\right)}\left\|R_{q}^{2}\right\|_{L_{T}^{m}\left(L^{2}\right)}^{2}\right)^{\frac{1}{2}}\right\} .
\end{aligned}
$$

Supposons tout d'abord que $\alpha \neq 1$. Comme $\alpha>0$, alors les lemmes B.3, B.5 et B.1 de [9], impliquent que

$$
\begin{aligned}
\left(\sum_{q \geq-1} 2^{2 q\left(s-2+\frac{2}{m}\right)} \| \Delta_{q}(a \operatorname{div} \mathcal{M})\right. & \left.-\operatorname{div}\left(a \Delta_{q} \mathcal{M}\right) \|_{L_{T}^{m}\left(L^{2}\right)}^{2}\right)^{\frac{1}{2}} \\
& \lesssim\|\nabla a\|_{\widetilde{L}_{T}^{\infty}\left(H^{\frac{N}{2}+\alpha-1}\right)}\|\nabla u\|_{\widetilde{L}_{T}^{m}\left(H^{s+\frac{2}{m}-1-\alpha \vee 1}\right)}
\end{aligned}
$$

et

$$
\begin{aligned}
\left(\sum_{q \geq-1} 2^{2 q\left(s-2+\frac{2}{m}\right)}\right. & \left.\left\|\mathcal{H}_{q}\right\|_{L_{T}^{m}\left(L^{2}\right)}^{2}\right)^{\frac{1}{2}} \\
& \lesssim\|\nabla a\|_{\widetilde{L}_{T}^{\infty}\left(H^{\frac{N}{2}+\alpha-1}\right)}\left\|\left(\widetilde{\mu}(a)-\mu^{1}\right) \mathcal{M}\right\|_{\widetilde{L}_{T}^{m}\left(H^{s+\frac{2}{m}-1-\alpha \vee 1}\right)},
\end{aligned}
$$


avec

$$
\mathcal{H}_{q}=\Delta_{q}\left[a \operatorname{div}\left\{\left(\widetilde{\mu}(a)-\mu^{1}\right) \mathcal{M}\right\}\right]-\operatorname{div}\left[a \Delta_{q}\left(\left\{\widetilde{\mu}(a)-\mu^{1}\right\} \mathcal{M}\right)\right],
$$

et par suite en utilisant le fait que $s+\frac{2}{m}-1-\alpha \vee 1 \leq \frac{N}{2}+\alpha$, la remarque 2.4 et l'inégalité (2.17), on obtient

$$
\begin{aligned}
\left(\sum_{q \geq-1} 2^{2 q\left(s-2+\frac{2}{m}\right)}\left\|\mathcal{H}_{q}\right\|_{L_{T}^{m}\left(L^{2}\right)}^{2}\right)^{\frac{1}{2}} & \\
& \lesssim\|\nabla a\|_{\widetilde{L}_{T}^{\infty}\left(H^{\frac{N}{2}+\alpha-1}\right)}\|a\|_{\widetilde{L}_{T}^{\infty}\left(H^{\frac{N}{2}+\alpha}\right)}\|\nabla u\|_{\tilde{L}_{T}^{m}\left(H^{s+\frac{2}{m}-1-\alpha \vee 1}\right)}
\end{aligned}
$$

donc

$$
\begin{aligned}
& \left(\sum_{q \geq-1} 2^{2 q\left(s+\frac{2}{m}-2\right)}\left\|R_{q}^{1}\right\|_{L_{T}^{m}\left(L^{2}\right)}^{2}\right)^{\frac{1}{2}} \\
& \quad \quad \lesssim\|\nabla a\|_{\widetilde{L}_{T}^{\infty}\left(H^{\frac{N}{2}+\alpha-1}\right)}\|\nabla u\|_{\widetilde{L}_{T}^{m}\left(H^{s+\frac{2}{m}-1-\alpha \vee 1}\right)}\left(\|a\|_{\widetilde{L}_{T}^{\infty}\left(H^{\frac{N}{2}+\alpha}\right)}+1\right) .
\end{aligned}
$$

De nouveau en utilisant le fait que $s+\frac{2}{m}-1-\alpha \vee 1 \leq \frac{N}{2}+\alpha$, alors l'inégalité de Hölder et l'inégalité (2.17) donnent

$$
\begin{aligned}
\left(\sum_{q \geq-1} 2^{2 q\left(s-1+\frac{2}{m}\right)}\left\|R_{q}^{2}\right\|_{L_{T}^{m}\left(L^{2}\right)}^{2}\right)^{\frac{1}{2}} & \lesssim\left\|\widetilde{\mu}(a)-\mu^{1}\right\|_{\widetilde{L}_{T}^{\infty}\left(H^{\frac{N}{2}+\alpha}\right)}\|\nabla u\|_{\widetilde{L}_{T}^{m}\left(H^{s+\frac{2}{m}-1-\alpha \vee 1}\right)} \\
& \lesssim\|a\|_{\widetilde{L}_{T}^{\infty}\left(H^{\frac{N}{2}+\alpha}\right)}\|\nabla u\|_{\widetilde{L}_{T}^{m}\left(H^{s+\frac{2}{m}-1-\alpha \vee 1}\right)} .
\end{aligned}
$$

Pour $\alpha=1$, il suffit de remplacer $\|\nabla a\|_{\widetilde{L}_{T}^{\infty}\left(H^{\frac{N}{2}}\right)}$ par $\|\nabla a\|_{\widetilde{L}_{T}^{\infty}\left(H^{\frac{N}{2}} \cap L^{\infty}\right)}$ pour la majoration de $R_{q}^{1}$ et $\|a\|_{\widetilde{L}_{T}^{\infty}\left(H^{\frac{N}{2}+1}\right)}$ par $\|a\|_{\widetilde{L}_{T}^{\infty}\left(H^{\frac{N}{2}+1}\right)}+\|\nabla a\|_{L_{T}^{\infty}\left(L^{\infty}\right)}$ dans l'estimation de $R_{q}^{2}$.

De même, on a

$$
\left(\sum_{q \geq-1} 2^{2 q s}\left\|\left[v, \Delta_{q}\right] \cdot \nabla u\right\|_{L_{T}^{1}\left(L^{2}\right)}^{2}\right)^{\frac{1}{2}} \lesssim \int_{0}^{T} V^{\prime}(t)\|u(t)\|_{H^{s}} d t
$$

avec (voir [9, lemme B.5])

$$
V^{\prime}(t) \stackrel{\text { déf }}{=} \begin{cases}\|\nabla v\|_{B_{2 \infty}^{\frac{N}{2}} \cap L^{\infty}} & \text { si }-\frac{N}{2}<s<\frac{N}{2}+1 \\ \|\nabla v\|_{H^{s-1}} & \text { si } s>\frac{N}{2}+1 \\ \|\nabla u(t)\|_{L^{\infty}} & \text { si } v=u \text { et } 0<s \\ 0 & \text { si } \nabla v=0 .\end{cases}
$$


Puisque $s+\frac{2}{m}-2 \leq \frac{N}{2}+\alpha$, alors d'après l'inégalité (2.15), la proposition 1.4 et la remarque 1.7 de [9], on a

$$
\begin{aligned}
\left\|T_{\nabla a} \Pi_{1}\right\|_{\widetilde{L}_{T}^{1}\left(H^{s}\right)} & \lesssim\|\nabla a\|_{\widetilde{L}_{T}^{\infty}\left(H^{\frac{N}{2}+\alpha-1}\right)}\left\|\nabla \Pi_{1}\right\|_{\widetilde{L}_{T}^{1}\left(H^{s-\alpha^{\prime}}\right)}, \\
\left\|T_{\nabla \Pi_{1}}^{\prime} a\right\|_{\widetilde{L}_{T}^{1}\left(H^{s}\right)} & \lesssim\|a\|_{\widetilde{L}_{T}^{\infty}\left(H^{\frac{N}{2}+\alpha}\right)}\left\|\nabla \Pi_{1}\right\|_{\widetilde{L}_{T}^{1}\left(H^{s-\alpha^{\prime}}\right)}, \\
\left\|T_{\nabla a} \Pi_{2}\right\|_{\widetilde{L}_{T}^{m}\left(H^{s+\frac{2}{m}-2}\right)} & \lesssim\|\nabla a\|_{\widetilde{L}_{T}^{\infty}\left(H^{\frac{N}{2}+\alpha-1}\right)}\left\|\nabla \Pi_{2}\right\|_{\widetilde{L}_{T}^{m}\left(H^{s+\frac{2}{m}-2-\alpha^{\prime}}\right)}, \\
\left\|T_{\nabla \Pi_{2}}^{\prime} a\right\|_{\widetilde{L}_{T}^{m}\left(H^{s+\frac{2}{m}-2}\right)} & \lesssim\|a\|_{\widetilde{L}_{T}^{\infty}\left(H^{\frac{N}{2}+\alpha}\right)}\left\|\nabla \Pi_{2}\right\|_{\widetilde{L}_{T}^{m}\left(H^{s+\frac{2}{m}-2-\alpha^{\prime}}\right)} .
\end{aligned}
$$

Comme $s>2-\frac{2}{m}$, alors pour $0<\alpha^{\prime} \leq \inf \left(1, \frac{1}{2} \alpha, \frac{s+\frac{2}{m}-2}{2}\right)$, on trouve grâce a la proposition A.5 de [9], les inégalités suivantes

$$
\begin{aligned}
\underline{a}\left\|\nabla \Pi_{1}\right\|_{\widetilde{L}_{T}^{1}\left(H^{s-\alpha^{\prime}}\right)} & \lesssim \mathcal{A}_{T}^{\frac{s+1}{\alpha^{\prime}}}\|\mathcal{Q} G\|_{\widetilde{L}_{T}^{1}\left(H^{s-\alpha^{\prime}}\right)} \\
& \lesssim \mathcal{A}_{T}^{\frac{s+1}{\alpha^{\prime}}}\left(\|\mathcal{Q} g\|_{\widetilde{L}_{T}^{1}\left(H^{s}\right)}+\int_{0}^{T} V^{\prime}(t)\|u(t)\|_{H^{s}} d s\right),
\end{aligned}
$$

avec

$$
\mathcal{A}_{T} \stackrel{\text { déf }}{=} \begin{cases}1+\underline{a}^{-1}\|\nabla a\|_{\widetilde{L}_{T}^{\infty}\left(H^{\frac{N}{2}+\alpha-1}\right)} & \text { si } \alpha \neq 1 \\ 1+\underline{a}^{-1}\|\nabla a\|_{\widetilde{L}_{T}^{\infty}\left(H^{\frac{N}{2}} \cap L^{\infty}\right)} & \text { si } \alpha=1\end{cases}
$$

Pour majorer $\left\|\nabla \Pi_{2}\right\|_{\widetilde{L}_{T}^{m}\left(H^{s+\frac{2}{m}-2-\alpha^{\prime}}\right)}$, il y a deux cas possibles soit $\alpha^{\prime} \leq s+$ $\frac{2}{m}-2-\alpha^{\prime} \leq \frac{N}{2}+\alpha^{\prime}$ soit $1 \leq s+\frac{2}{m}-2-\alpha^{\prime} \leq \frac{N}{2}+\alpha$, et par suite

$$
\begin{aligned}
& \underline{a}\left\|\nabla \Pi_{2}\right\|_{\widetilde{L}_{T}^{m}\left(H^{s+\frac{2}{m}-2-\alpha^{\prime}}\right)} \lesssim \mathcal{A}_{T}^{\frac{s+\frac{2}{m}-2}{\alpha^{\prime}}}\|\mathcal{Q} H\|_{\widetilde{L}_{T}^{m}\left(H^{s+\frac{2}{m}-2-\alpha^{\prime}}\right)} \lesssim \mathcal{A}_{T}^{\frac{s+\frac{2}{m}-2}{\alpha^{\prime}}} \\
& \quad \times\left\{\|a \Delta u\|_{\widetilde{L}_{T}^{m}\left(H^{s+\frac{2}{m}-2-\alpha^{\prime}}\right)}+\left\|(1+a) \operatorname{div}\left[\left(\widetilde{\mu}(a)-\mu^{1}\right) \mathcal{M}\right]\right\| \|_{\widetilde{L}_{T}^{m}\left(H^{s+\frac{2}{m}-2-\alpha^{\prime}}\right)}\right\} .
\end{aligned}
$$

Puisque $s+\frac{2}{m}-2-\alpha^{\prime} \leq \frac{N}{2}+\alpha$, alors la remarque 2.4 et les inégalités de Bernstein et (2.17) impliquent

$$
\begin{aligned}
& \underline{a}\left\|\nabla \Pi_{2}\right\|_{\widetilde{L}_{T}^{m}\left(H^{s+\frac{2}{m}-2-\alpha^{\prime}}\right)} \\
& \quad \lesssim \mathcal{A}_{T}^{\frac{s+\frac{2}{m}-2}{\alpha^{\prime}}}\|a\|_{\widetilde{L}_{T}^{\infty}\left(H^{\frac{N}{2}+\alpha}\right)}\|u\|_{\widetilde{L}_{T}^{m}\left(H^{s+\frac{2}{m}-\alpha^{\prime}}\right)}\left\{\|a\|_{\widetilde{L}_{T}^{\infty}\left(H^{\frac{N}{2}+\alpha}\right)}+1\right\} .
\end{aligned}
$$


Donc

$$
\begin{aligned}
\|u\|_{\widetilde{L}_{T}^{\infty}\left(H^{s}\right)} & +\bar{\mu}^{\frac{1}{m}}\|u\|_{\widetilde{L}_{T}^{m}\left(H^{s+\frac{2}{m}}\right)} \lesssim\left\|u_{0}\right\|_{H^{s}}+\bar{\mu}^{\frac{1}{m}}\left\|\Delta_{-1} u\right\|_{L_{T}^{m}\left(L^{2}\right)}+\|\mathcal{P} g\|_{\widetilde{L}_{T}^{1}\left(H^{s}\right)} \\
& +\mathcal{A}_{T}^{\frac{s+1}{\alpha^{\prime}}}\|a\|_{\widetilde{L}_{T}^{\infty}\left(H^{\frac{N}{2}+\alpha}\right)}\left(\|\mathcal{Q} g\|_{\widetilde{L}_{T}^{1}\left(H^{s}\right)}+\int_{0}^{T} V^{\prime}(t)\|u(t)\|_{H^{s}} d t\right) \\
& +\bar{\mu}^{\frac{1}{m}-1} \mathcal{A}_{T}^{\frac{s+\frac{2}{m}-2}{\alpha^{\prime}}}\|a\|_{\widetilde{L}_{T}^{\infty}\left(H^{\frac{N}{2}+\alpha}\right)}^{3}\|u\|_{\widetilde{L}_{T}^{m}\left(H^{s+\frac{2}{m}-\alpha^{\prime}}\right)} \\
& +\bar{\mu}^{\frac{1}{m}} \mathcal{A}_{T}\|u\|_{\widetilde{L}_{T}^{m}\left(H^{s+\frac{2}{m}-\alpha^{\prime}}\right)}\|a\|_{\widetilde{L}_{T}^{\infty}\left(H^{\frac{N}{2}+\alpha}\right)}+\int_{0}^{T} V^{\prime}(t)\|u(t)\|_{H^{s}} d t
\end{aligned}
$$

Soit

$$
\mathcal{B}_{T} \stackrel{\text { déf }}{=} \begin{cases}1+\|a\|_{\widetilde{L}_{T}^{\infty}\left(H^{\frac{N}{2}+\alpha}\right)} & \text { si } \alpha \neq 1 \\ 1+\|a\|_{\widetilde{L}_{T}^{\infty}\left(H^{\frac{N}{2}+1}\right)}+\|\nabla a\|_{L_{T}^{\infty}\left(L^{\infty}\right)} & \text { si } \alpha=1\end{cases}
$$

alors

$$
\begin{aligned}
\|u\|_{\widetilde{L}_{T}^{\infty}\left(H^{s}\right)}+\bar{\mu}^{\frac{1}{m}}\|u\|_{\widetilde{L}_{T}^{m}\left(H^{\left.s+\frac{2}{m}\right)}\right.} \lesssim & \left\|u_{0}\right\|_{H^{s}}+\bar{\mu}^{\frac{1}{m}} \mathcal{B}_{T}^{\frac{s+\frac{2}{m}+2}{\alpha^{\prime}}+\left[\frac{N}{2}+\alpha\right]}\|u\|_{\widetilde{L}_{T}^{m}\left(H^{s+\frac{2}{m}-\alpha^{\prime}}\right)} \\
& +\mathcal{B}_{T}^{\frac{s+2}{\alpha^{\prime}}}\left(\|g\|_{\widetilde{L}_{T}^{1}\left(H^{s}\right)}+\int_{0}^{T} V^{\prime}(t)\|u(t)\|_{H^{s}} d t\right) .
\end{aligned}
$$

Pour conclure il suffit d'utiliser le lemme de Gronwall.

Remarque 4.1. Remarquons que pour s vérifiant $s+\frac{2}{m}-1 \leq \frac{N}{2}+\alpha$, on a $\nabla \Pi_{2}$ à coefficients dans $\widetilde{L}_{T}^{m}\left(H^{s+\frac{2}{m}-2}\right)$. De plus

$$
\underline{a}\left\|\nabla \Pi_{2}\right\|_{\widetilde{L}_{T}^{m}\left(H^{s+\frac{2}{m}-2}\right)} \lesssim \mathcal{A}_{T}^{\frac{s}{\alpha^{\prime}}}\|a\|_{\widetilde{L}_{T}^{\infty}\left(H^{\frac{N}{2}+\alpha}\right)}\|u\|_{\widetilde{L}_{T}^{m}\left(H^{s+\frac{2}{m}}\right)}\left(\mu^{1}+\|a\|_{\widetilde{L}_{T}^{\infty}\left(H^{\frac{N}{2}+\alpha}\right)}\right) .
$$

Grâce à la méthode de Friedrichs et la proposition 4.1, on obtient le résultat suivant (la démonstration est la même que celle de la proposition 3.5 de $[9])$.

Proposition 4.2. Soient $T>0$ et $\mu, m, s, \alpha, \alpha^{\prime}, u_{0}, g, a, v$ vérifiant les conditions de la proposition 4.1. On suppose de plus que $\left(\partial_{t}+v \cdot \nabla\right) \log (1+a)$ $\in L_{T}^{1}\left(L^{\infty}\left(\mathbb{R}^{N}\right)\right)$. Alors le système $(M)$ admet une unique solution $(u, \nabla \Pi)$ telle que

$$
\begin{aligned}
& u \in\left(\widetilde{C}_{T}\left(H^{s}\left(\mathbb{R}^{N}\right)\right)\right)^{N}, \quad \bar{\mu}^{\frac{1}{m}} u \in\left(\widetilde{L}_{T}^{m}\left(H^{s+\frac{2}{m}}\left(\mathbb{R}^{N}\right)\right)\right)^{N} \\
& \text { et } \nabla \Pi \in\left(\widetilde{L}_{T}^{1}\left(H^{s}\left(\mathbb{R}^{N}\right)\right)\right)^{N}+\left(\widetilde{L}_{T}^{m}\left(H^{s+\frac{2}{m}-2-\alpha^{\prime}}\left(\mathbb{R}^{N}\right)\right)\right)^{N} .
\end{aligned}
$$


Enfin pour démontrer que le système $(\widetilde{\mathrm{INS}})$ est localement bien posé dans $H^{s}$, pour $s$ suffisamment grand, il suffit d'utiliser le schéma itératif et la proposition 4.1, (pour plus de détaille voir la fin de preuve du théorème 0.2 de [9]). D'où le théorème 1.2.

Le théorème 1.2 nous permet démontre le résultat d'existence dans l'espace $\dot{B}_{p, 1}^{\frac{N}{p}} \times \dot{B}_{p, 1}^{\frac{N}{p}-1}$ pour $1<p<2 N$. Notons que dans ce cas $u_{0}$ est dans un espace à régularité négative. En d'autres termes, nous avons.

Théorème 4.1. Soient $1<p<2 N$ et $\mu$ vérifie (1.1). Il existe une constante $c$ dépendant de $N, p$ et de la fonction $\mu$ telle que pour $u_{0}$ un champ de vecteurs à divergence nulle avec coefficients dans $\dot{B}_{p 1}^{\frac{N}{p}-1}\left(\mathbb{R}^{N}\right), f$ appartenant $\grave{a}\left(L^{1}\left(\mathbb{R}_{+} ; B_{p 1}^{\frac{N}{p}-1}\left(\mathbb{R}^{N}\right)\right)\right)^{N}$ avec $\mathcal{Q} f \in\left(L_{\text {loc }}^{2}\left(\mathbb{R}_{+} ; \dot{B}_{p 1}^{\frac{N}{p}-2}\left(\mathbb{R}^{N}\right)\right)\right)^{N}$ et $a_{0} \in$ $\dot{B}_{p 1}^{\frac{N}{p}}\left(\mathbb{R}^{N}\right)$ où

$$
\left\|a_{0}\right\|_{\dot{B}_{p 1}^{\frac{N}{p}}} \leq c
$$

alors il existe $T \in(0,+\infty]$ tel que le système $(\widetilde{\mathrm{INS}})$ admette une solution $(a, u, \nabla \Pi)$ vérifiant

$$
\begin{gathered}
a \in C_{b}\left([0, T) ; \dot{B}_{p 1}^{\frac{N}{p}}\right) \cap \widetilde{L}_{T}^{\infty}\left(\dot{B}_{p 1}^{\frac{N}{p}}\right), \quad u \in\left(C_{b}\left([0, T) ; \dot{B}_{p 1}^{\frac{N}{p}-1}\right) \cap L_{T}^{1}\left(\dot{B}_{p 1}^{\frac{N}{p}+1}\right)\right)^{N} \\
\text { et } \nabla \Pi \in\left(L_{T}^{1}\left(\dot{B}_{p 1}^{\frac{N}{p}-1}\right)\right)^{N} .
\end{gathered}
$$

De plus il existe une constante $\beta$ dépendant de $N, p$ et $\mu$ telle que

$$
\|a\|_{\widetilde{L}_{T}^{\infty}\left(\dot{B_{p}}\right)} \leq \beta\left\|a_{0}\right\|_{\dot{B}_{p 1}^{\frac{N}{p}}}
$$

et

$\|u\|_{\widetilde{L}_{T}^{\infty}\left(\dot{B}_{p 1}^{\frac{N}{p}-1}\right)}+\mu^{1}\|u\|_{L_{T}^{1}\left(\dot{B}_{p 1}^{\frac{N}{p}}+1\right.}+\|\nabla \Pi\|_{L_{T}^{1}\left(\dot{B}_{p 1}^{\frac{N}{p}}-1\right.} \leq \beta\left(\left\|u_{0}\right\|_{\dot{B}_{p 1}^{\frac{N}{p}-1}}+\|f\|_{L_{T}^{1}\left(\dot{B}_{p 1}^{\frac{N}{p}-1}\right)}\right)$.

De plus $\nabla \Pi \in\left(L_{T}^{\frac{2}{2-\eta}}\left(\dot{B}_{p 1}^{\frac{N}{p}-1-\eta}\right)\right)^{N}$ avec $0 \leq \eta<\inf \left(1, \frac{2 N-p}{2 p}\right)$ et $T$ peut être minoré par

$$
\begin{aligned}
\max \left\{t \geq 0 \mid\|\mathcal{Q} f\|_{L_{t}^{1}\left(\dot{B}_{p 1}^{\frac{N}{p}-1}\right)}+\sum_{q \in \mathbb{Z}} 2^{q\left(\frac{N}{p}-1\right)}\right. & \left(\left\|\dot{\Delta}_{q} u_{0}\right\|_{L^{p}}+\left\|\dot{\Delta}_{q} \mathcal{P} f\right\|_{L_{t}^{1}\left(L^{p}\right)}\right) \\
& \left.\times\left(\frac{1-e^{-K \mu^{1} t 2^{2 q}}}{K}\right) \leq \frac{c\left(\mu^{1}\right)^{2}}{\mu^{1}+U_{0}}\right\},
\end{aligned}
$$

avec $\mu^{1}=\mu(1)$ et $U_{0} \stackrel{\text { déf }}{=}\left\|u_{0}\right\|_{\dot{B}_{p 1}^{\frac{N}{p}-1}}+\|\mathcal{P} f\|_{L^{1}\left(\mathbb{R}_{+} ; \dot{B}_{p 1}^{\frac{N}{p}-1}\right)}$. 


\subsection{Démonstration du théorème 4.1}

La démonstration du théorème s'effectue en trois étapes. La première consiste à résoudre un problème approché, la deuxième étape à démontrer que la solution du problème approché est uniformément bornée et la dernière, à étudier la convergence de la solution approchée et démontrer que sa limite est une solution de $(\widetilde{\mathrm{INS}})$.

\section{Première étape : Construction d'une solution approchée régulière}

Tout d'abord on démontre que l'ensemble des fonctions régulières est dense dans l'espace $\dot{B}_{p r}^{s}$.

Lemme 4.2. Soient $1 \leq i \leq 4, s_{i} \in \mathbb{R},\left(p_{i}, r_{i}\right) \in\left[1, \infty\left[^{2}\right.\right.$ et $(G, H, F) \in$ $\dot{B}_{p_{1} r_{1}}^{s_{1}}\left(\mathbb{R}^{N}\right) \times \dot{B}_{p_{2} r_{2}}^{s_{2}}\left(\mathbb{R}^{N}\right) \times \dot{B}_{p_{3} r_{3}}^{s_{3}}\left(\mathbb{R}^{N}\right)$, telles que $\operatorname{div} H=0$ et $\mathcal{Q} F \in \dot{B}_{p_{4} r_{4}}^{s_{4}}$. Alors pour tout $\varepsilon>0$ il existe une suite $\left(G_{\varepsilon}\right)_{\varepsilon \geq 0}$ régulière et $n_{0}$ tel que

$$
\left\|G_{\varepsilon}-G\right\|_{\dot{B}_{p_{1} r_{1}}^{s_{1}}} \leq \varepsilon \quad \text { pour tout } n \geq n_{0} .
$$

Si de plus $G \in L^{\infty}\left(\mathbb{R}^{N}\right)$, alors

$$
\left\|G_{\varepsilon}\right\|_{L^{\infty}\left(\mathbb{R}^{N}\right)} \lesssim\|G\|_{L^{\infty}\left(\mathbb{R}^{N}\right)} .
$$

De même pour $H$ et $F$ avec de plus $H_{\varepsilon} \in H^{\infty}\left(\mathbb{R}^{N}\right)$ et $F_{\varepsilon} \in H^{\infty}\left(\mathbb{R}^{N}\right)$ telles que $\operatorname{div} H_{\varepsilon}=0$ et $\mathcal{Q} F_{\varepsilon}$ bornée dans $\dot{B}_{p_{3} r_{3}}^{s_{4}}$.

Preuve. On régularise $G$ par l'opérateur $\dot{\Delta}_{q}$ et on localise : pour $n \in \mathbb{N}$ et $0<\delta<1$, on définit

$$
G^{n, \delta} \stackrel{\text { déf }}{=} \Theta_{\delta} \sum_{|q| \leq n} \dot{\Delta}_{q} G
$$

avec $\Theta_{\delta}(x)=\Theta(\delta x)$ telle que $\Theta \in \mathcal{S}\left(\mathbb{R}^{N}\right), \Theta(0)=1$, supp $\mathcal{F} \Theta \subset B(0,1)$. Pour simplifier les notations, on pose $G_{\varepsilon} \stackrel{\text { déf }}{=} G^{n, \delta}$.

Tout d'abord l'inégalité de Bernstein montre que $G_{\varepsilon} \in \mathcal{S}\left(\mathbb{R}^{N}\right)$. Pour montrer la convergence, on commence par faire tendre $\delta$ vers 0 , pour cela on prouve que $G_{\varepsilon} \rightarrow \sum_{|q| \leq n} \dot{\Delta}_{q} G$ dans $\dot{B}_{p r}^{s}$ quand $\delta \rightarrow 0$ : en effet par définition de l'opérateur $\dot{\Delta}_{k}$, on a

$$
\begin{aligned}
\dot{\Delta}_{k} G_{\varepsilon}(x) & -\dot{\Delta}_{k}\left(\sum_{|q| \leq n} \dot{\Delta}_{q} G\right)(x) \\
& =2^{k N} \int_{\mathbb{R}^{N}} h\left(2^{N}(x-y)\right)\left(\Theta(\delta y) \sum_{|q| \leq n} \dot{\Delta}_{q} G(y)-\sum_{|q| \leq n} \dot{\Delta}_{q} G(y)\right) d y .
\end{aligned}
$$


Comme

$$
\Theta(\delta y) \sum_{|q| \leq n} \dot{\Delta}_{q} G(y)-\sum_{|q| \leq n} \dot{\Delta}_{q} G(y) \rightarrow 0 \quad \text { pour tout } y \text { quand } \delta \rightarrow 0,
$$

$\sum_{|q| \leq n} \dot{\Delta}_{q} G \in L^{p}, h \in L^{p^{\prime}}$ et $\left\|\Theta_{\delta}\right\|_{L^{\infty}} \lesssim 1$ alors le théorème de Lebesgue implique que

$$
\dot{\Delta}_{k} G_{\varepsilon}(x)-\dot{\Delta}_{k}\left(\sum_{|q| \leq n} \dot{\Delta}_{q} G\right)(x) \rightarrow 0 \quad \text { quand } \delta \rightarrow 0
$$

et

$$
\left|\dot{\Delta}_{k} G_{\varepsilon}(x)-\dot{\Delta}_{k}\left(\sum_{|q| \leq n} \dot{\Delta}_{q} G\right)(x)\right| \lesssim \int_{\mathbb{R}^{N}}\left|h\left(2^{N}(x-y)\right)\right|\left|\sum_{|q| \leq n} \dot{\Delta}_{q} G(y)\right| d y
$$

appartient à $L^{p}$ d'après Young. Le théorème de Lebesgue implique alors que

$$
\left\|\dot{\Delta}_{k} G^{n}(x)-\dot{\Delta}_{k}\left(\sum_{|q| \leq n} \dot{\Delta}_{q} G\right)(x)\right\|_{L^{p}} \rightarrow 0 \quad \text { quand } \quad \delta \rightarrow 0 .
$$

D'autre part comme supp $\mathcal{F} \Theta_{\delta} \subset B(0, \delta)$ et

$$
\operatorname{supp} \mathcal{F}\left(\sum_{|q| \leq n} \dot{\Delta}_{q} G\right) \subset C\left(0, \frac{3}{4} 2^{-n}, \frac{8}{3} 2^{n}\right),
$$

alors pour $\delta \leq \frac{3}{8} 2^{-n}$, on a

$$
\operatorname{supp} \mathcal{F} G_{\varepsilon} \subset C\left(0, \frac{3}{8} 2^{-n}, \frac{11}{3} 2^{n}\right)
$$

et par suite

$$
\dot{\Delta}_{k}\left(\Theta_{\delta} \sum_{|q| \leq n} \dot{\Delta}_{q} G\right) \equiv 0 \quad \text { si } k-n \geq 3 \quad \text { ou } n+k \leq-3 .
$$

D'autre part

$$
\left\|\dot{\Delta}_{k}\left(\Theta_{\delta} \sum_{|q| \leq n} \dot{\Delta}_{q} G\right)\right\|_{L^{p}} \lesssim \sum_{|q| \leq n}\left\|\dot{\Delta}_{q} G\right\|_{L^{p}}
$$

et

$$
\sum_{|k| \leq n+2} 2^{k s r} \sum_{|q| \leq n}\left\|\dot{\Delta}_{q} G\right\|_{L^{p}}<\infty,
$$

donc le théorème de Lebesgue implique que

$$
\left\|G_{\varepsilon}-\sum_{|q| \leq n} \dot{\Delta}_{q} G\right\|_{\dot{B}_{p r}^{s}} \rightarrow 0 \quad \text { quand } \delta \rightarrow 0 .
$$


Maintenant on va démontrer que $\sum_{|q| \leq n} \dot{\Delta}_{q} G \rightarrow G$ dans $\dot{B}_{p r}^{s}$ quand $n \rightarrow \infty$. Mais $G-\sum_{|q| \leq n} \dot{\Delta}_{q} G=\sum_{|q| \geq n+1} \dot{\Delta}_{q} G \rightarrow 0$ dans $\dot{B}_{p r}^{s}$ quand $n \rightarrow \infty$, car $\sum_{q} \dot{\Delta}_{q} G \in \dot{B}_{p r}^{s}$. D'où $\mathcal{S}\left(\mathbb{R}^{n}\right) \cap \dot{B}_{p r}^{s}\left(\mathbb{R}^{N}\right)$ dense dans $\dot{B}_{p r}^{s}\left(\mathbb{R}^{N}\right)$. Pour montrer que $G_{\varepsilon}$ est bornée dans $L^{\infty}\left(\mathbb{R}^{N}\right)$, premièrement d'après l'inégalité de Hölder, on a

$$
\left\|G_{\varepsilon}\right\|_{L^{\infty}\left(\mathbb{R}^{N}\right)} \leq\left\|\sum_{|q| \leq n} \dot{\Delta}_{q} G\right\|_{L^{\infty}\left(\mathbb{R}^{N}\right)}\left\|\Theta_{\delta}\right\|_{L^{\infty}\left(\mathbb{R}^{N}\right)} \lesssim\left\|\sum_{|q| \leq n} \dot{\Delta}_{q} G\right\|_{L^{\infty}\left(\mathbb{R}^{N}\right)},
$$

mais $\sum_{|q| \leq n} \dot{\Delta}_{q} G=\dot{S}_{n+1} G-\dot{S}_{-n} G$, on utilise le fait que l'opérateur $\dot{S}_{q}$ est continu sur les espaces de Lebesgue, on trouve

$$
\left\|\sum_{|q| \leq n} \dot{\Delta}_{q} G\right\|_{L^{\infty}} \lesssim\|G\|_{L^{\infty}}
$$

Pour $H$ il suffit de prendre $H_{\varepsilon}=\mathcal{P}\left(\Theta_{\delta} \sum_{|q| \leq n} \dot{\Delta}_{q} H\right)$ qui appartient à $H^{\infty}\left(\mathbb{R}^{N}\right)$ car $\Theta_{\delta} \sum_{|q| \leq n} \dot{\Delta}_{q} H \in\left(\mathcal{S}\left(\mathbb{R}^{N}\right)\right)^{N}$ d'après l'inégalité de Bernstein et par suite a ses coefficients dans $H^{\infty}\left(\mathbb{R}^{N}\right)$. Enfin, on utilise le fait que $\mathcal{P}$ est continue sur les espace de Sobolev et de Besov homogène.

On approche $F$ par

$$
F_{\varepsilon}=\mathcal{P}\left(\Theta_{\delta} \sum_{|q| \leq n} \dot{\Delta}_{q} F\right)+\Theta_{\delta} \sum_{|q| \leq n} \dot{\Delta}_{q} \mathcal{Q} F
$$

De même on a $F_{\varepsilon} \in H^{\infty}\left(\mathbb{R}^{N}\right)$, et on utilise le fait que $\mathcal{P}$ est continue sur l'espace de Besov homogène. Donc on a

$$
\mathcal{P}\left(\Theta_{\delta} \sum_{|q| \leq n} \dot{\Delta}_{q} F\right) \rightarrow \mathcal{P} \sum_{|q| \leq n} \dot{\Delta}_{q} F \quad \text { quand } \delta \rightarrow 0 \text { dans } \dot{B}_{p_{3} r_{3}}^{s_{3}}
$$

et comme $\mathcal{Q}$ est continue sur l'epace de Besov homogène, alors

$$
\Theta_{\delta} \sum_{|q| \leq n} \dot{\Delta}_{q} \mathcal{Q} F \rightarrow \sum_{|q| \leq n} \dot{\Delta}_{q} \mathcal{Q} F \quad \text { quand } \delta \rightarrow 0 \text { dans } \dot{B}_{p_{3} r_{3}}^{s_{3}}
$$

et par suite

$$
\mathcal{P}\left(\Theta_{\delta} \sum_{|q| \leq n} \dot{\Delta}_{q} F\right)+\Theta_{\delta} \sum_{|q| \leq n} \dot{\Delta}_{q} \mathcal{Q} F \rightarrow \sum_{|q| \leq n} \dot{\Delta}_{q} F \quad \text { quand } \delta \rightarrow 0 \text { dans } \dot{B}_{p_{3}}^{s_{3}} r_{3} .
$$

D'autre part, par construction, on a $\mathcal{Q} F_{\varepsilon}=\mathcal{Q}\left(\Theta_{\delta} \sum_{|q| \leq n} \dot{\Delta}_{q} \mathcal{Q} F\right)$. De nouveau, la continuité de $\mathcal{Q}$ sur l'espace de Besov homogène implique que

$$
\left\|\mathcal{Q} F_{\varepsilon}\right\|_{\dot{B}_{p_{4} r_{4}}^{s_{4}}} \lesssim\|\mathcal{Q} F\|_{\dot{B}_{p_{4} r_{4}}^{s_{4}}} .
$$


D'après le lemme 4.2 il existe donc $a_{0}^{n} \in H^{s_{0}+1}\left(\mathbb{R}^{N}\right), u_{0}^{n} \in\left(H^{s_{0}}\left(\mathbb{R}^{N}\right)\right)^{N}$ et $f^{n} \in\left(\widetilde{L}_{\text {loc }}^{1}\left(\mathbb{R}_{+} ; H^{s_{0}}\left(\mathbb{R}^{N}\right)\right)\right)^{N}$ pour $s_{0}>\frac{N}{2}-1$ telles que $\left\|a_{0}^{n}\right\|_{L^{\infty}} \lesssim\left\|a_{0}\right\|_{L^{\infty}}, \operatorname{div} u_{0}^{n}=0$ et $\left\|\mathcal{Q} f^{n}\right\|_{L_{l o c}^{1}\left(\mathbb{R}_{+} ; \dot{B}_{p 1}^{\frac{N}{p}-2}\right)} \lesssim\|\mathcal{Q} f\|_{L_{l o c}^{1}\left(\mathbb{R}_{+} ; \dot{B}_{p 1}^{\frac{N}{p}}-2\right.}$.

Maintenant grâce au théorème 1.2 on peut déduire que le système ( $\widetilde{\mathrm{INS}})$ avec données $\left(a_{0}^{n}, u_{0}^{n}, f^{n}\right)$ admet une solution locale unique $\left(a^{n}, u^{n}, \nabla \Pi^{n}\right)$ telle que

$$
\begin{gathered}
a^{n} \in C\left(\left[0, T^{n}\right) ; H^{s_{0}+1}\left(\mathbb{R}^{N}\right)\right), u^{n} \in\left(C\left(\left[0, T^{n}\right) ; H^{s_{0}}\left(\mathbb{R}^{N}\right)\right)\right)^{N} \\
\text { et } \nabla \Pi^{n} \in\left(\widetilde{L}^{1}\left(\left[0, T^{n}\right) ; H^{s_{0}}\left(\mathbb{R}^{N}\right)\right)\right)^{N} .
\end{gathered}
$$

\section{Deuxième étape : Estimation de la solution régularisée}

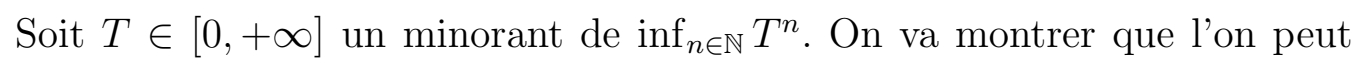
choisir $T>0$ tel que $\left(a^{n}, u^{n}, \nabla \Pi^{n}\right)$ appartienne à et soit uniformément bornée dans

$$
E_{T}=\widetilde{L}_{T}^{\infty}\left(\dot{B}_{p 1}^{\frac{N}{p}}\right) \times\left(L_{T}^{1}\left(\dot{B}_{p 1}^{\frac{N}{p}+1}\right) \cap \widetilde{L}_{T}^{\infty}\left(\dot{B}_{p 1}^{\frac{N}{p}-1}\right)\right)^{N} \times\left(L_{T}^{1}\left(\dot{B}_{p 1}^{\frac{N}{p}-1}\right)\right)^{N} .
$$

Soit $\left(u_{L}^{n}, \Pi_{L}^{n}\right)$ la solution du système de Stokes non-stationnaire suivant

$$
(\mathrm{L}) \quad\left\{\begin{array}{l}
\partial_{t} u_{L}^{n}-\mu^{1} \Delta u_{L}^{n}+\nabla \Pi_{L}^{n}=f^{n} \\
\operatorname{div} u_{L}^{n}=0 \\
u_{L \mid t=0}^{n}=u_{0}^{n}
\end{array}\right.
$$

Par construction,

$$
u_{0}^{n} \in \dot{B}_{p 1}^{\frac{N}{p}-1}\left(\mathbb{R}^{N}\right) \cap H^{s_{0}}\left(\mathbb{R}^{N}\right) \quad \text { et } \quad f^{n} \in\left(\widetilde{L}_{l o c}^{1}\left(\mathbb{R}_{+} ; \dot{B}_{p 1}^{\frac{N}{p}-1} \cap H^{s_{0}}\right)\right)^{N} .
$$

Donc, d'après la proposition 2.3 [10], on a

$$
\left(u_{L}^{n}, \nabla \Pi_{L}^{n}\right) \in\left(L_{T}^{\infty}\left(\dot{B}_{p 1}^{\frac{N}{p}-1} \cap H^{s_{0}}\right)\right)^{N} \times\left(\widetilde{L}_{T}^{1}\left(\dot{B}_{p 1}^{\frac{N}{p}-1} \cap H^{s_{0}}\right)\right)^{N}
$$

et de plus $u_{L}^{n} \in\left(\widetilde{L}_{T}^{1}\left(\dot{B}_{p 1}^{\frac{N}{p}+1} \cap H^{s_{0}+1}\right)\right)^{N}$ pour tout $T>0$.

On décompose $\left(u^{n}, \nabla \Pi^{n}\right)$ sous la forme suivante $u^{n}=u_{L}^{n}+\bar{u}^{n}$ et $\nabla \Pi^{n}=$ $\nabla \Pi_{L}^{n}+\nabla \bar{\Pi}^{n}$. Alors

$$
\left(a^{n}, \bar{u}^{n}, \nabla \bar{\Pi}^{n}\right) \in C\left(\left[0, T^{n}\right) ; H^{s_{0}+1}\right) \times\left(C\left(\left[0, T^{n}\right) ; H^{s_{0}}\right)\right)^{N} \times\left(\widetilde{L}_{T^{n}}^{1}\left(H^{s_{0}}\right)\right)^{N}
$$

satisfait

$$
(\mathrm{NL})\left\{\begin{array}{l}
\partial_{t} a^{n}+u^{n} \cdot \nabla a^{n}=0 \\
\partial_{t} \bar{u}^{n}+u^{n} \cdot \nabla \bar{u}^{n}-\mu^{1} \Delta \bar{u}^{n}+\nabla \bar{\Pi}^{n}=H\left(a^{n}, u^{n}, \nabla \Pi^{n}\right) \\
\operatorname{div} \bar{u}^{n}=0 \\
\left(a^{n}, \bar{u}^{n}\right)_{\mid t=0}=\left(a_{0}^{n}, 0\right),
\end{array}\right.
$$


avec

$$
\begin{aligned}
H\left(a^{n}, u^{n}, \nabla \Pi^{n}\right)= & -u^{n} \cdot \nabla u_{L}^{n}+a^{n}\left(\mu^{1} \Delta u^{n}-\nabla \Pi^{n}\right) \\
& +\left(1+a^{n}\right) \operatorname{div}\left\{\left(\widetilde{\mu}\left(a^{n}\right)-\mu^{1}\right) \mathcal{M}^{n}\right\}
\end{aligned}
$$

et $\mathcal{M}^{n}=\nabla u^{n}+{ }^{t} \nabla u^{n}$. Nous allons montrer dans la suite que $\left(a^{n}, \bar{u}^{n}, \nabla \bar{\Pi}^{n}\right) \in$ $E_{T^{n}}$. Remarquons que le problème se pose seulement pour $p<2$ et les basses fréquences. Dans le cas $2 \leq p<2 N$ et $N \geq 3$ on utilise le fait que

$$
\begin{aligned}
\left(a^{n}, \bar{u}^{n}, \nabla \bar{\Pi}^{n}\right) \in C\left(\left[0, T^{n}\right) ; H^{s_{0}+1}\left(\mathbb{R}^{N}\right)\right) & \times\left(C\left(\left[0, T^{n}\right) ; H^{s_{0}}\left(\mathbb{R}^{N}\right)\right)\right)^{N} \\
& \times\left(\widetilde{L}^{1}\left(\left[0, T^{n}\right) ; H^{s_{0}}\left(\mathbb{R}^{N}\right)\right)\right)^{N}
\end{aligned}
$$

et l'inégalité de Bernstein, qui entraîne $H^{s_{0}} \hookrightarrow \dot{B}_{p 1}^{s}\left(\mathbb{R}^{N}\right)$ dès que $s_{0}>s+$ $\frac{N}{2}-\frac{N}{p}$ (on a besoin de ca pour les hautes fréquences) et $s>\frac{N}{p}-\frac{N}{2}$ puisque pour les basses fréquences, on a

$$
\sum_{q \leq-1} 2^{s q}\left\|\Delta_{q} h\right\|_{L^{p}} \lesssim \sum_{q \leq-1} 2^{q\left(s+\frac{N}{2}-\frac{N}{p}\right)}\left\|\Delta_{q} h\right\|_{L^{2}} \lesssim\|h\|_{L^{2}}
$$

Donc $\left(a^{n}, u^{n}, \nabla \Pi^{n}\right) \in E_{T^{n}}$ pour $2 \leq p<2 N$ et $N \geq 3$. Pour $1<p<2$ ou $N=2$, on utilise l'inégalité $(2.8)$, le fait que $H^{s_{0}}\left(\mathbb{R}^{N}\right) \hookrightarrow \dot{B}_{2 r}^{s}\left(\mathbb{R}^{N}\right)$ dès que $s_{0}>s$ et $s>0$ et le résultat suivant qui donne une majoration des solutions de système de Stokes non stationnaire dans les espaces de Besov homogènes construits sur les espaces de Lebesgue $L^{p}$ par ceux construits sur $L^{2}$.

Lemme 4.3. Soient $p \in] 1, \infty\left[, 0<s+1+N-\frac{N}{p}, r \in[1,+\infty], u_{0}\right.$ un champ de vecteurs à divergence nulle avec coefficients dans $\dot{B}_{p r}^{s}$ et $g$ un champ de vecteurs à coefficients dans $\widetilde{L}_{T}^{1}\left(\dot{B}_{p r}^{s}\right)$. Soient $u$ et $v$ des champs de vecteurs à divergence nulle tels que $v \in\left(L_{T}^{1}\left(\dot{B}_{2 r}^{s+1+N-\frac{N}{p}} \cap L^{2}\right)\right)^{N}$ et $u \in$ $\left(L_{T}^{\infty}\left(\dot{B}_{2 r}^{s+1+N-\frac{N}{p}} \cap L^{2}\right)\right)^{N}$ une solution de système de Stokes non stationnaire

$$
(\mathcal{L}) \quad\left\{\begin{array}{l}
\partial_{t} u+v \cdot \nabla u-\nu \Delta u+\nabla \Pi=g \\
\operatorname{div} u=0 \\
u_{\mid t=0}=u_{0} .
\end{array}\right.
$$

Alors il existe $C$ dépendant de $N$, $p$ et s tel que u vérifie l'estimation suivante

$$
\begin{aligned}
& \|u\|_{\widetilde{L}_{T}^{\infty}\left(\dot{B}_{p r}^{s}\right)}+\nu\|u\|_{\widetilde{L}_{T}^{1}\left(\dot{B}_{p r}^{s+2}\right)}+\|\nabla \Pi\|_{\widetilde{L}_{T}^{1}\left(\dot{B}_{p r}^{s}\right)} \\
& \quad \leq C\left(\left\|u_{0}\right\|_{\dot{B}_{p r}^{s}}+\|g\|_{\widetilde{L}_{T}^{1}\left(\dot{B}_{p r}^{s}\right)}+\|u\|_{L_{T}^{\infty}\left(L^{2} \cap \dot{B}_{2 r}^{s+1+N-\frac{N}{p}}\right)}\|v\|_{L_{T}^{1}\left(L^{2} \cap \dot{B}_{2 r}^{s+1+N-\frac{N}{p}}\right)}\right) .
\end{aligned}
$$


Preuve. La démonstration s'appuie sur celle de la proposition 3.3 et l'inégalité (2.8). En effet : on a

$$
\partial_{t} u-\nu \Delta u+\nabla \Pi=g-v \cdot \nabla u .
$$

En reprenant mot pour mot la preuve de la proposition 3.3 et en utilisant l'inégalite de Bernstein, on obtient

$$
\begin{aligned}
\|u\|_{\widetilde{L}_{T}^{\infty}\left(\dot{B}_{p r}^{s}\right)}+\nu\|u\|_{\widetilde{L}_{T}^{1}\left(\dot{B}_{p r}^{s+2}\right)} & +\|\nabla \Pi\|_{\widetilde{L}_{T}^{1}\left(\dot{B}_{p r}^{s}\right)} \\
& \leq\left\|u_{0}\right\|_{\dot{B}_{p r}^{s}}+C\|g\|_{\widetilde{L}_{T}^{1}\left(\dot{B}_{p r}^{s}\right)}+C \int_{0}^{t}\|v \cdot \nabla u\|_{\dot{B}_{p r}^{s}} d \tau \\
& \leq\left\|u_{0}\right\|_{\dot{B}_{p r}^{s}}+C\|g\|_{\widetilde{L}_{T}^{1}\left(\dot{B}_{p r}^{s}\right)}+C \int_{0}^{t}\|v \otimes u\|_{\dot{B}_{p r}^{s+1}} d \tau .
\end{aligned}
$$

Comme $s+1+N-\frac{N}{p}>0$, alors les inégalites (2.8) et de Hölder impliquent

$$
\begin{aligned}
& \|u\|_{\widetilde{L}_{T}^{\infty}\left(\dot{B}_{p r}^{s}\right)}+\nu\|u\|_{\widetilde{L}_{T}^{1}\left(\dot{B}_{p r}^{s+2}\right)}+\|\nabla \Pi\|_{\widetilde{L}_{T}^{1}\left(\dot{B}_{p r}^{s}\right)} \\
& \quad \leq\left\|u_{0}\right\|_{\dot{B}_{p r}^{s}}+C\|g\|_{\widetilde{L}_{T}^{1}\left(\dot{B}_{p r}^{s}\right)}+C\|u\|_{L_{T}^{\infty}\left(L^{2} \cap \dot{B}_{2 r}^{\left.s+1+N-\frac{N}{p}\right)}\right.}\|v\|_{L_{T}^{1}\left(L^{2} \cap \dot{B}_{2 r}^{s+1+N-\frac{N}{p}}\right)} .
\end{aligned}
$$

De même on a une estimation analogue pour l'équation de transport, plus exactement on a pour $s+1+N-\frac{N}{p}>0$

$$
\begin{aligned}
\|f\|_{\widetilde{L}_{T}^{\infty}\left(\dot{B}_{p r}^{s}\right)} \leq\left\|f_{0}\right\|_{\dot{B}_{p r}^{s}}+\int_{0}^{t}\|F(\tau)\|_{\dot{B}_{p r}^{s}} d \tau & +C\|f\|_{L_{T}^{\infty}\left(L^{2} \cap \dot{B}_{2 r}^{s+1+N-\frac{N}{p}}\right)} \\
& \times\|v\|_{L_{T}^{1}\left(L^{2} \cap \dot{B}_{2 r}^{s+1+N-\frac{N}{p}}\right)}
\end{aligned}
$$

Appliquons le lemme 4.3 avec $u=\bar{u}^{n}, v=u^{n}, F=0$,

$g=-u^{n} \cdot \nabla u_{L}^{n}+a^{n}\left(\mu^{1} \Delta u^{n}-\nabla \Pi^{n}\right)+\left(1+a^{n}\right) \operatorname{div}\left\{\left(\widetilde{\mu}\left(a^{n}\right)-\mu^{1}\right) \mathcal{M}^{n}\right\}$ et $s=\frac{N}{p}-1$ et l'inégalité (2.8) que implique que

$$
\left\|a^{n} \Delta u^{n}\right\|_{\dot{B}_{p 1}^{\frac{1}{p}-1}} \lesssim\left\|a^{n}\right\|_{\dot{B}_{21}^{N} \cap L^{2}}\left\|\Delta u^{n}\right\|_{\dot{B}_{p 1}^{N} \cap L^{2}} \lesssim\left\|a^{n}\right\|_{H^{s_{0}}}\left\|\Delta u^{n}\right\|_{H^{s_{0}}} \text { si } s_{0}>N \text {. }
$$

On en déduit que $\left(a^{n}, u^{n}, \nabla \Pi^{n}\right) \in E_{T^{n}}$ pour $1<p$.

Maintenant on va démontrer que $\left(a^{n}, u^{n}, \nabla \Pi^{n}\right)$ est bornée dans $E_{T^{n}}$.

D'après la proposition 3.2 , on a

$$
\begin{aligned}
\left\|a^{n}\right\|_{\widetilde{L}_{T^{n}}^{\infty}\left(\dot{B}_{p 1}^{\frac{N}{p}}\right)} & \leq \exp \left(C\left\|\nabla u^{n}\right\|_{\left.L_{T^{n}\left(\dot{B}_{p 1}^{\frac{N}{p}}\right)}\right)\left\|a_{0}^{n}\right\|_{\dot{B}_{p 1}^{\frac{N}{p}}}} \leq \alpha \exp \left(C\left\|\nabla u^{n}\right\|_{\left.L_{T^{n}\left(\dot{B}_{p 1}^{1}\right)}^{\frac{N}{p}}\right)}\right)\left\|a_{0}\right\|_{\dot{B}_{p 1}^{\frac{N}{p}}},\right.
\end{aligned}
$$

où $\alpha \stackrel{\text { déf }}{=} c\|\tilde{h}\|_{L^{1}}$ (plus exactement $c$ dépend de la fonction $\Theta$ du lemme 4.2). 
De plus la proposition 3.3 implique que

$$
\begin{aligned}
& \left\|\bar{u}^{n}\right\|_{\widetilde{L}_{T^{n}}^{\infty}\left(\dot{B}_{p 1}^{\frac{N}{p}-1}\right)}+\mu^{1}\left\|\bar{u}^{n}\right\|_{L_{T^{n}}^{1}\left(\dot{B}_{p 1}^{\frac{N}{p}+1}\right)}+\left\|\nabla \bar{\Pi}^{n}\right\|_{\left.L_{T^{n}\left(\dot{B}_{p 1}^{\frac{N}{p}}-1\right.}\right)} \\
& \leq C \exp \left(C\left\|\nabla u^{n}\right\|_{L_{T^{n}}^{1}\left(\dot{B}_{p 1}^{\frac{N}{p}}\right)}\right)\left\|H\left(a^{n}, u^{n}, \nabla \Pi^{n}\right)\right\|_{L_{T^{n}}^{1}\left(\dot{B}_{p 1}^{\frac{N}{p}-1}\right)} .
\end{aligned}
$$

D'après l'inégalité (2.12), on a

$$
\begin{aligned}
\left\|-u^{n} \cdot \nabla u_{L}^{n}+a^{n}\left(\mu^{1} \Delta u^{n}-\nabla \Pi^{n}\right)\right\|_{L_{T^{n}}^{1}\left(\dot{B}_{p 1}^{\frac{N}{p}-1}\right)} \\
\qquad\left\|u^{n}\right\|_{\widetilde{L}_{T^{n}}^{\infty}\left(\dot{B}_{p 1}^{\frac{N}{p}-1}\right)}\left\|\nabla u_{L}^{n}\right\|_{L_{T^{n}}^{1}\left(\dot{B}_{p 1}^{\frac{N}{p}}\right)} \\
\quad+\left\|a^{n}\right\|_{L_{T^{n}}^{\infty}\left(\dot{B}_{p \infty}^{\frac{N}{p}} \cap L^{\infty}\right)}\left(\left\|u^{n}\right\|_{L_{T^{n}}^{1}\left(\dot{B}_{p 1}^{\frac{N}{p}+1}\right)}+\left\|\nabla \Pi^{n}\right\|_{L_{T^{n}}^{1}\left(\dot{B}_{p 1}^{\frac{N}{p}-1}\right)}\right) .
\end{aligned}
$$

Les inégalités (2.12), Bernstein, (2.11) et (2.17) impliquent

$$
\begin{aligned}
\|\left(1+a^{n}\right) \operatorname{div} & \left\{\left(\widetilde{\mu}\left(a^{n}\right)-\mu^{1}\right) \mathcal{M}^{n}\right\} \|_{L_{T^{n}}^{1}\left(\dot{B}_{p 1}^{\frac{N}{p}-1}\right)} \\
& \lesssim\left(1+\left\|a^{n}\right\|_{L_{T^{n}}^{\infty}\left(\dot{B}_{p \infty}^{\frac{N}{p}} \cap L^{\infty}\right)}\right)\left\|\left(\widetilde{\mu}\left(a^{n}\right)-\mu^{1}\right) \mathcal{M}^{n}\right\|_{\left.L_{T^{n}\left(\dot{B}_{p 1}^{1}\right)}^{\frac{N}{p}}\right)} \\
& \lesssim\left(1+\left\|a^{n}\right\|_{L_{T^{n}}^{\infty}\left(\dot{B}_{p \infty}^{\frac{N}{p}} \cap L^{\infty}\right)}\right)\left\|a^{n}\right\|_{\widetilde{L}_{T^{n}}^{\infty}\left(\dot{B}_{p 1}^{\frac{N}{p}}\right)} \mu^{1}\left\|u^{n}\right\|_{L_{T^{n}}^{1}\left(\dot{B}_{p 1}^{\frac{N}{p}+1}\right)}
\end{aligned}
$$

On en déduit que

$$
\begin{aligned}
& \left\|\bar{u}^{n}\right\|_{\widetilde{L}_{T^{n}}^{\infty}\left(\dot{B}_{p 1}^{\frac{N}{p}}-1\right)}+\mu^{1}\left\|\bar{u}^{n}\right\|_{L_{T^{n}}^{1}\left(\dot{B}_{p 1}^{\frac{N}{p}}+1\right)}+\left\|\nabla \bar{\Pi}^{n}\right\|_{L_{T^{n}}^{1}\left(\dot{B}_{p 1}^{\frac{N}{p}}-1\right.} \\
& \leq C \exp \left(C\left\|\nabla u^{n}\right\|_{L_{T^{n}}^{1}\left(\dot{B}_{p 1}^{\frac{N}{p}}\right)}\right)\left[\left\|u^{n}\right\|_{\left.\widetilde{L}_{T^{n}\left(\dot{B}_{p 1}^{\infty}\right.}^{\frac{N}{p}-1}\right)}\left\|\nabla u_{L}^{n}\right\|_{L_{T^{n}}^{1}\left(\dot{B}_{p 1}^{\frac{N}{p}}\right)}+\left\|a^{n}\right\|_{\widetilde{L}_{T^{n}}^{\infty}\left(\dot{B}_{p 1}^{\frac{N}{p}}\right)}\right. \\
& \left.\times\left(1+\left\|a^{n}\right\|_{L_{T^{n}}^{\infty}\left(\dot{B}_{p}^{p} \cap L^{\infty}\right)}\right)\left(\mu^{1}\left\|u^{n}\right\|_{L_{T^{n}}^{1}\left(\dot{B}_{p 1}^{\frac{N}{p}}+1\right.}^{p}+\left\|\nabla \Pi^{n}\right\|_{\left.L_{T^{n}\left(\dot{B}_{p 1}^{1}\right.}^{\frac{N}{p}-1}\right)}\right)\right] .
\end{aligned}
$$

Soit $\zeta$ un réel strictement positif petit. Il existe alors $T_{1}>0$ tel que

$$
\mu^{1}\left\|u_{L}\right\|_{L_{T_{1}}^{1}\left(\dot{B}_{p 1}^{\frac{N}{p}+1}\right)}+\left\|\nabla \Pi_{L}\right\|_{L_{T_{1}}^{1}\left(\dot{B}_{p 1}^{\frac{N}{p}-1}\right)} \leq \zeta
$$

En effet, on applique l'opérateur $\mathcal{Q}$ sur $(L)$, on obtient

$$
\left\|\nabla \Pi_{L}\right\|_{L_{T_{1}}^{1}\left(\dot{B}_{p 1}^{\frac{N}{p}-1}\right)} \leq\|\mathcal{Q} f\|_{L_{T_{1}}^{1}\left(\dot{B}_{p 1}^{\frac{N}{p}-1}\right)} \leq\|f\|_{L_{T_{1}}^{1}\left(\dot{B}_{p 1}^{\frac{N}{p}-1}\right)} \leq \zeta
$$


pour $T_{1}$ petit car $f \in L_{l o c}^{1}\left(\mathbb{R}_{+} ; \dot{B}_{p 1}^{\frac{N}{p}-1}\right)$ et

$$
\begin{aligned}
& \mu^{1}\left\|u_{L}\right\|_{L_{t}^{1}\left(\dot{B}_{p 1}^{\frac{N}{p}+1}\right)} \\
& \leq C\left\{\sum_{q \in \mathbb{Z}} 2^{q\left(\frac{N}{p}-1\right)}\left(\left\|\dot{\Delta}_{q} u_{0}\right\|_{L^{p}}+\left\|\dot{\Delta}_{q} \mathcal{P} f\right\|_{L_{t}^{1}\left(L^{p}\right)}\right)\left(\frac{1-e^{-K \mu^{1} t 2^{2 q}}}{K}\right)\right\} \\
& \stackrel{\text { déf }}{=} F(t) \leq \zeta,
\end{aligned}
$$

pour $t$ assez petit car le théorème de Lebesgue implique $t \longmapsto F(t)$ est continue et clairement $F(0)=0$. De même la proposition 2.3 de [10] implique

$$
\left\|u_{L}\right\|_{\widetilde{L}_{T_{1}}^{\infty}\left(\dot{B}_{p 1}^{\frac{N}{p}-1}\right)} \leq\left\|u_{0}\right\|_{\dot{B}_{p 1}^{\frac{N}{p}-1}}+\|\mathcal{P} f\|_{L_{T_{1}}^{1}\left(\dot{B}_{p 1}^{\frac{N}{p}-1}\right)} \stackrel{\text { déf }}{=} U_{0} .
$$

Et par suite

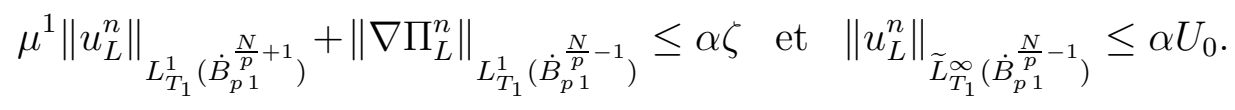

Dans la suite on peut supposer que $T^{n} \leq T_{1}$ ( sinon on diminue $T^{n}$ ), donc pour $t \leq T^{n}$

$$
\begin{aligned}
& \left\|\bar{u}^{n}\right\|_{\widetilde{L}_{t}^{\infty}\left(\dot{B}_{p 1}^{\frac{N}{p}-1}\right)}+\mu^{1}\left\|\bar{u}^{n}\right\|_{L_{t}^{1}\left(\dot{B}_{p 1}^{\frac{N}{p}+1}\right)}+\left\|\nabla \bar{\Pi}^{n}\right\|_{L_{t}^{1}\left(\dot{B}_{p 1}^{\frac{N}{p}-1}\right)}
\end{aligned}
$$

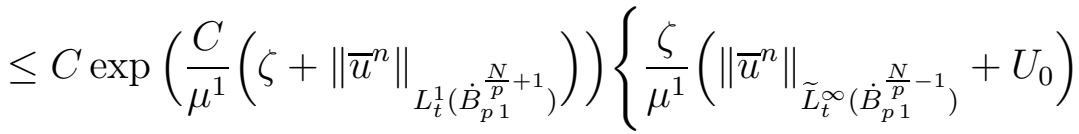

$$
\begin{aligned}
& +\left\|a^{n}\right\|_{\widetilde{L}_{t}^{\infty}\left(\dot{B}_{p 1}^{\frac{N}{p}}\right)}\left(1+\left\|a^{n}\right\|_{L_{t}^{\infty}\left(\dot{B}_{p 1}^{\frac{N}{p}}\right)}\right) \\
& \left.\times\left(\zeta+\mu^{1}\left\|\bar{u}^{n}\right\|_{L_{t}^{1}\left(\dot{B}_{p 1}^{\frac{N}{p}+1}\right)}+\left\|\nabla \bar{\Pi}^{n}\right\|_{L_{t}^{1}\left(\dot{B}_{p 1}^{\frac{N}{p}-1}\right)}\right)\right\}
\end{aligned}
$$

et

$$
\left\|a^{n}\right\|_{\widetilde{L}_{t}^{\infty}\left(\dot{B}_{p 1}^{\frac{N}{p}}\right)} \leq \alpha \exp \left(\frac{C}{\mu^{1}}\left(\zeta+\left\|\bar{u}^{n}\right\|_{L_{t}^{1}\left(\dot{B}_{p 1}^{\frac{N}{p}+1}\right)}\right)\right)\left\|a_{0}\right\|_{\dot{B}_{p 1}^{\frac{N}{p}}} .
$$

Donc si

$$
8 C\left\|a_{0}\right\|_{\dot{B}_{p 1}^{\frac{N}{p}}}\left(1+2\left\|a_{0}\right\|_{\dot{B}_{p 1}^{\frac{N}{p}}}\right) \leq 1 \quad \text { et } \quad \frac{C \zeta}{\mu^{1}}\left(1+\frac{2 C U_{0}}{\mu^{1}}\right) \leq \frac{1}{8},
$$

alors

$$
\begin{aligned}
& \left\|\bar{u}^{n}\right\|_{\widetilde{L}_{t}^{\infty}\left(\dot{B}_{p 1}^{\frac{N}{p}-1}\right)}+\mu^{1}\left\|\bar{u}^{n}\right\|_{L_{t}^{1}\left(\dot{B}_{p 1}^{\frac{N}{p}+1}\right)}+\left\|\nabla \bar{\Pi}^{n}\right\|_{L_{t}^{1}\left(\dot{B}_{p 1}^{\frac{N}{p}-1}\right)} \leq \zeta\left(2+\frac{8 C U_{0}}{\mu^{1}}\right), \\
& \left\|a^{n}\right\|_{\widetilde{L}_{t}^{\infty}\left(\dot{B}_{p 1}^{\frac{N}{p}}\right)} \leq 2 \alpha\left\|a_{0}\right\|_{\dot{B}_{p 1}^{\frac{N}{p}}}
\end{aligned}
$$


En effet : soit

$$
U^{n}(t) \stackrel{\text { déf }}{=}\left\|\bar{u}^{n}\right\|_{\widetilde{L}_{t}^{\infty}\left(\dot{B}_{p 1}^{\frac{N}{p}-1}\right)}+\mu^{1}\left\|\bar{u}^{n}\right\|_{L_{t}^{1}\left(\dot{B}_{p 1}^{\frac{N}{p}+1}\right)}+\left\|\nabla \bar{\Pi}^{n}\right\|_{L_{t}^{1}\left(\dot{B}_{p 1}^{\frac{N}{p}-1}\right)} .
$$

Choissions $T_{2}$ tel que

$$
\exp \left(\frac{C}{\mu^{1}}\left(\zeta+\left\|\bar{u}^{n}\right\|_{L_{T_{2}}^{1}\left(\dot{B}_{p 1}^{\frac{N}{p}+1}\right)}\right)\right) \leq 2 .
$$

Les inégalités (4.9) et (4.10) impliquent que

$$
U^{n}\left(T_{2}\right) \leq 2 C\left\{\frac{\zeta}{\mu^{1}}\left(U^{n}\left(T_{2}\right)+U_{0}\right)+2\left\|a_{0}\right\|_{\dot{B}_{p 1}^{\frac{N}{p}}}\left(1+\left\|a_{0}\right\|_{\dot{B}_{p 1}^{\frac{N}{p}}}\right)\left(\zeta+U^{n}\left(T_{2}\right)\right)\right\},
$$

et par suite

$$
U^{n}\left(T_{2}\right) \leq \zeta\left(2+\frac{8 C U_{0}}{\mu^{1}}\right) \quad \text { et } \quad\left\|a^{n}\right\|_{\widetilde{L}_{T_{2}}^{\infty}\left(\dot{B} \dot{B}_{p 1}^{\frac{N}{p}}\right)} \leq 2 \alpha\left\|a_{0}\right\|_{\dot{B}_{p 1}^{\frac{N}{p}}} .
$$

Lorsque $\xi$ suffisamment petite, linégalité (4.12) est vérifier. Et par suite par méthode standard de connexité on montre que $T_{2}=T^{n}$. Le même raisonnement permet d'avoir $T^{n}=T^{1}$, avec des contrôles uniformes.

Lemme 4.4. Soient $1<p<2 N$ et $0<\eta<\inf \left(1, \frac{2 N-p}{2 p}\right)$. Alors $\left(\nabla \Pi^{n}\right)_{n \in \mathbb{N}}$ est uniformément bornée dans $\left(L_{T_{1}}^{\frac{2}{2-\eta}}\left(\dot{B}_{p 1}^{\frac{N}{p}-1-\eta}\right)\right)^{N}$.

Preuve. Pour majorer la pression, on utilise le fait que

$$
\operatorname{div}\left(\left(1+a^{n}\right) \nabla \Pi^{n}\right)=\operatorname{div}\left(\mathcal{Q} f^{n}-u^{n} \cdot \nabla u^{n}+\left(1+a^{n}\right) \operatorname{div}\left\{\widetilde{\mu}\left(a^{n}\right) \mathcal{M}^{n}\right\}\right) .
$$

Par construction de $f^{n}$ et l'inégalité (2.10), $\mathcal{Q} f^{n}$ est uniformément bornée dans $\left(L_{T_{1}}^{\frac{2}{2-\eta}}\left(\dot{B}_{p 1}^{\frac{N}{p}-1-\eta}\right)\right)^{N}$. De nouveau l'inégalité (2.10) implique que $u^{n}$ est uniformément bornée dans $\left(L_{T_{1}}^{\frac{2}{1-\eta}}\left(\dot{B}_{p 1}^{\frac{N}{p}-\eta}\right)\right)^{N}$, et par suite le choix de $\eta, p<2 N$ et l'inégalité (2.5), on a $u^{n} \cdot \nabla u^{n}$ uniformément bornée dans

$$
\left(L_{T_{1}}^{\frac{2}{2-\eta}}\left(\dot{B}_{p 1}^{\frac{N}{p}-1-\eta}\right)\right)^{N}
$$

Enfin pour $\left(1+a^{n}\right) \operatorname{div}\left\{\widetilde{\mu}\left(a^{n}\right) \mathcal{M}^{n}\right\}$, les inégalités (2.3) et (2.5) impliquent $\operatorname{div}\left\{\widetilde{\mu}\left(a^{n}\right) \mathcal{M}^{n}\right\}$ est uniformément bornée dans

$$
\left(L_{T_{1}}^{1}\left(\dot{B}_{p r}^{\frac{N}{p}-1}\right)\right)^{N} \cap\left(L_{T_{1}}^{2}\left(\dot{B}_{p 1}^{\frac{N}{p}-2}\right)\right)^{N} .
$$


Et par suite, par interpolation, $\operatorname{div}\left\{\widetilde{\mu}\left(a^{n}\right) \mathcal{M}^{n}\right\}$, est uniformément bornée dans $\left(L_{T_{1}}^{\frac{2}{2-\eta}}\left(\dot{B}_{p 1}^{\frac{N}{p}-1-\eta}\right)\right)^{N}$. Alors l'inégalité $(2.5)$ implique que

$$
\left(1+a^{n}\right) \operatorname{div}\left\{\widetilde{\mu}\left(a^{n}\right)\left(\nabla u^{n}+{ }^{t} \nabla u^{n}\right)\right\}
$$

est uniformément bornée dans $\left(L_{T_{1}}^{\frac{2}{2-\eta}}\left(\dot{B}_{p 1}^{\frac{N}{p}-1-\eta}\right)\right)^{N}$, donc $\nabla \Pi^{n}$ aussi car

$$
\left\|a^{n}\right\|_{\widetilde{L}_{T_{1}}^{\infty}\left(\dot{B}_{p 1}^{\frac{N}{p}}\right)} \leq 2 \alpha\left\|a_{0}\right\|_{\dot{B}_{p 1}^{\frac{N}{p}}}<<1
$$

D'autre part les inégalités (4.6) et (4.7) montrent que si

$$
U_{0}+\|\mathcal{Q} f\|_{L^{1}\left(\mathbb{R}_{+} ; \dot{B}_{p 1}^{\frac{N}{p}-1}\right)} \leq c^{\prime} \mu^{1}, \quad \text { alors } \quad T_{1}=+\infty .
$$

\section{Troisième étape : Convergence}

Tout d'abord par construction de $\left(u_{0}^{n}, f^{n}\right)$, par définition de $\left(u_{L}^{n}, \nabla \Pi_{L}^{n}\right)$ et unicité des solutions du système $(L)$, le couple $\left(u_{L}^{n}, \nabla \Pi_{L}^{n}\right)$ converge vers la solution $\left(u_{L}, \nabla \Pi_{L}\right)$, du système $(L)$. Par contre pour montrer que la limite de $\left(a^{n}, \bar{u}^{n}, \nabla \bar{\Pi}^{n}\right)$ est une solution du système $(N L)$, il va falloir recourir à des arguments de compacité.

Nous savons que $\left(a^{n}, \bar{u}^{n}, \nabla \bar{\Pi}^{n}\right)$ est uniformément bornée dans

$$
\widetilde{L}_{T_{1}}^{\infty}\left(\dot{B}_{p 1}^{\frac{N}{p}}\right) \times\left(\widetilde{L}_{T_{1}}^{\infty}\left(\dot{B}_{p 1}^{\frac{N}{p}-1}\right) \cap L_{T_{1}}^{1}\left(\dot{B}_{p 1}^{\frac{N}{p}+1}\right)\right)^{N} \times\left(L_{T_{1}}^{1}\left(\dot{B}_{p 1}^{\frac{N}{p}-1}\right)\right)^{N},
$$

et que de plus $\nabla \Pi^{n}$ est uniformément bornée dans $\left(L_{T_{1}}^{\frac{2}{2-\eta}}\left(\dot{B}_{p 1}^{\frac{N}{p}-1-\eta}\right)\right)^{N}$.

Alors pour pouvoir utiliser le théorème d'Ascoli, il suffit d'estimer la dérivée par rapport au temps de $a^{n}$ et $\bar{u}^{n}$ (voir par exemple [11]).

\section{Lemme 4.5.}

(i) La suite $\left(\partial_{t} a^{n}\right)_{n \in \mathbb{N}}$ est uniformément bornée dans $L_{T_{1}}^{2}\left(\dot{B}_{p r}^{\frac{N}{p}-1}\right)$.

(ii) La suite $\left(\partial_{t} \bar{u}^{n}\right)_{n \in \mathbb{N}}$ est uniformément bornée dans $\left(L_{T_{1}}^{\frac{2}{2-\eta}}\left(\dot{B}_{p 1}^{\frac{N}{p}-1-\eta}\right)\right)^{N}$ pour $0<\eta<\inf \left(1, \frac{2 N-p}{2 p}\right)$.

Preuve. Rappelons que

$$
\partial_{t} a^{n}=-u^{n} \cdot \nabla a^{n} .
$$

Comme $p<2 N$ alors grâce à l'inégalité (2.5) et par interpolation, on trouve

$$
\begin{aligned}
& \left.\left\|\partial_{t} a^{n}\right\|_{L_{T_{1}}^{2}\left(\dot{B}_{p r}^{\frac{N}{p}}-1\right)} \lesssim\left\|u^{n}\right\|_{L_{T_{1}}^{2}\left(\dot{B}_{p 1}^{\frac{N}{p}}\right)}\left\|\nabla a^{n}\right\|_{L_{T_{1}}^{\infty}\left(\dot{B}_{p r}^{\frac{N}{p}}-1\right.}\right) \\
& \lesssim\left\|u^{n}\right\|_{L_{T_{1}}^{\infty}\left(\dot{B}_{p 1}^{\frac{N}{p}-1}\right)}^{\frac{1}{2}}\left\|u^{n}\right\|_{L_{T_{1}}^{1}\left(\dot{B}_{p 1}^{\frac{N}{p}+1}\right)}^{\frac{1}{2}}\left\|a^{n}\right\|_{L_{T_{1}}^{\infty}\left(\dot{B}_{p r}^{\frac{N}{p}}\right)} .
\end{aligned}
$$


Pour $\bar{u}^{n}$, on a

$\partial_{t} \bar{u}^{n}=-\mathcal{P}\left(u^{n} \cdot \nabla u^{n}\right)-\mu^{1} \Delta u_{L}^{n}-\mathcal{P}\left(a^{n} \nabla \Pi^{n}\right)+\mathcal{P}\left[\left(1+a^{n}\right) \operatorname{div}\left\{\widetilde{\mu}\left(a^{n}\right) \mathcal{M}^{n}\right\}\right]$.

Comme l'opérateur de Leray est continu sur les espaces de Besov homogènes, alors les calculs du lemme 4.4 restent valables. Il reste à montrer que $\mu^{1} \Delta u_{L}^{n}$ est uniformément borné dans $\left(L_{T_{1}}^{\frac{2}{2-\eta}}\left(\dot{B}_{p 1}^{\frac{N}{p}-1-\eta}\right)\right)^{N}$. Mais il est uniformément bornée dans

$$
\left(L_{T_{1}}^{1}\left(\dot{B}_{p 1}^{\frac{N}{p}-1}\right)\right)^{N} \cap\left(L_{T_{1}}^{\infty}\left(\dot{B}_{p 1}^{\frac{N}{p}-3}\right)\right)^{N} .
$$

Donc l'inégalité (2.10) implique que $\mu^{1} \Delta u_{L}^{n}$ est uniformément borné dans $\left(L_{T_{1}}^{\frac{2}{2-\eta}}\left(\dot{B}_{p 1}^{\frac{N}{p}-1-\eta}\right)\right)^{N}$.

À partir des inégalités de Cauchy-Schwarz et de Hölder et du lemme précédent, on obtient le corollaire suivant.

\section{Corollaire 4.1.}

(i) La suite $\left(a^{n}\right)_{n \in \mathbb{N}}$ est uniformément bornée dans $C^{\frac{1}{2}}\left(\left[0, T_{1}\right] ; \dot{B}_{p 1}^{\frac{N}{p}-1}\right)$.

(ii) La suite $\left(\bar{u}^{n}\right)_{n \in \mathbb{N}}$ est uniformément bornée dans $C^{\frac{\eta}{2}}\left(\left[0, T_{1}\right] ; \dot{B}_{p 1}^{\frac{N}{p}-2}\right)$ pour tout $\eta$ appartenant $\grave{a}] 0, \inf \left(1, \frac{2 N-p}{2 p}\right)[$.

Rappelons que

$$
\dot{B}_{p q, l o c}^{s+\varepsilon} \hookrightarrow B_{p q, l o c}^{s+\varepsilon} \hookrightarrow B_{p q, l o c}^{s}
$$

avec la deuxième injection compacte pour tout $\varepsilon>0$ (voir par exemple [22]). Il existe une sous-suite notée encore $\left(a^{n}, \bar{u}^{n}, \nabla \bar{\Pi}^{n}\right)$ qui converge vers $(a, \bar{u}, \nabla \bar{\Pi})$. Et par suite la proposition 2.2 et l'inégalité (4.13) assurent que $(a, u, \nabla \Pi)$ est une solution du système ( $\widetilde{\mathrm{INS}})$ et appartenant à

$$
\widetilde{L}_{T_{1}}^{\infty}\left(\dot{B}_{p 1}^{\frac{N}{p}}\right) \times\left(\widetilde{L}_{T_{1}}^{\infty}\left(\dot{B}_{p 1}^{\frac{N}{p}-1}\right) \cap L_{T_{1}}^{1}\left(\dot{B}_{p 1}^{\frac{N}{p}+1}\right)\right)^{N} \times\left(L_{T_{1}}^{1}\left(\dot{B}_{p 1}^{\frac{N}{p}-1}\right)\right)^{N} .
$$

Concernant la continuité de $u$ voir [11]. Pour montrer que $a$ est continue et que sa norme $L^{\infty}$ est conservée, on utilise que $a=a_{0} \circ \Psi^{-1}$ avec $\Psi$, flot de $u$, ce qui démontre le théorème 4.1 .

Remarque 4.2. Pour prouver l'unicité, on a eu seulement besoin d'avoir $a_{0}$ petite dans $\dot{B}_{p \infty}^{\frac{N}{p}} \cap L^{\infty}$ pour $3 \leq N$ et $1 \leq p<N$ et petite dans $\dot{B}_{p 1}^{1}$ sinon. Par contre pour l'existence cette condition est insuffisante, car le terme

$$
a^{n} \operatorname{div}\left\{\left(\widetilde{\mu}\left(a^{n}\right)-\mu^{1}\right)\left(\nabla \bar{u}^{n}+{ }^{t} \nabla \bar{u}^{n}\right)\right\}
$$

ne peut être absorbé dans les estimations que si $a^{n}$ (et donc $a_{0}$ ) est petit dans $\dot{B}_{p 1}^{\frac{N}{p}}$. 
En revanche si $\mu$ est constante, les termes nécessitant l'usage de la norme $\dot{B}_{p 1}^{\frac{N}{p}}$ pour $a^{n}$ sont nuls. Alors il suffit de prendre $a_{0} \in \dot{B}_{p}^{\frac{N}{p}} \cap L^{\infty}$ avec norme petite. Plus précisement on a le résultat suivant :

Corollaire 4.2. Soient $r \in[1, \infty[$ si $N>p$ et $N \geq 3, r=1$ si $p=N$ ou $N=2$ et $1<p \leq N$ (l'existence est valable pour $1<p<2 N$ ). Il existe une constante $c$ dépendant de $N$ et $p$ telle que pour

$$
\begin{aligned}
& u_{0} \in\left(\dot{B}_{p 1}^{\frac{N}{p}-1}\left(\mathbb{R}^{N}\right)\right)^{N} \quad \text { avec } \quad \operatorname{div} u_{0}=0, \\
& f \in\left(L^{1}\left(\mathbb{R}_{+} ; B_{p 1}^{\frac{N}{p}-1}\left(\mathbb{R}^{N}\right)\right)\right)^{N} \quad \text { avec } \quad \mathcal{Q} f \in\left(L_{l o c}^{2}\left(\mathbb{R}_{+} ; \dot{B}_{p 1}^{\frac{N}{p}-2}\left(\mathbb{R}^{N}\right)\right)\right)^{N} \\
\text { et } & a_{0} \in \dot{B}_{p r}^{\frac{N}{p}}\left(\mathbb{R}^{N}\right) \cap L^{\infty}\left(\mathbb{R}^{N}\right)
\end{aligned}
$$

où

$$
\left\|a_{0}\right\|_{\dot{B}_{p r}^{\frac{N}{p}} \cap L^{\infty}} \leq c \quad\left(\left\|a_{0}\right\|_{\dot{B}_{p 1}^{\frac{N}{p}}} \leq c \quad \text { si } \quad N=p \quad \text { ou } \quad N=2\right),
$$

alors il existe $T \in(0,+\infty]$ tel que le système $(\widetilde{\mathrm{INS}})$ admette une unique solution

$$
\begin{aligned}
(a, u, \nabla \Pi) \in C_{b}\left([0, T) ; \dot{B}_{p r}^{\frac{N}{p}}\right) & \cap L^{\infty}\left(0, T ; L^{\infty}\right) \times\left(C_{b}\left([0, T) ; \dot{B}_{p 1}^{\frac{N}{p}-1}\right)\right)^{N} \\
& \cap\left(L^{1}\left(0, T ; \dot{B}_{p 1}^{\frac{N}{p}+1}\right)\right)^{N} \times\left(L^{1}\left(0, T ; \dot{B}_{p 1}^{\frac{N}{p}-1}\right)\right)^{N} .
\end{aligned}
$$

De plus si

$$
\left\|u_{0}\right\|_{\dot{B}_{p 1}^{\frac{N}{p}-1}}+\|f\|_{L^{1}\left(\mathbb{R}_{+} ; \dot{B}_{p 1}^{\frac{N}{p}-1}\right)} \leq c \mu^{1}
$$

alors $T=+\infty$.

\section{Références}

[1] Antontsev, S., Kazhikhov, A. And Monakhov, V.: Boundary value problems in mechanics of nonhomogeneous fluids. Studies in mathematics and its applications 22. North-Holland, Amsterdam, 1990.

[2] Bahouri, H and Chemin, J.-Y.: Équations d'ondes quasilinénaires et estimations de Strichartz. Amer. J. Math. 121 (1999), no. 6, 1337-1377.

[3] Bony, J.-M.: Calcul symbolique et propagation des singularités pour les équations aux dérivées partielles non linéaires. Ann. Sci. École Norm. Sup. (4) 14 (1981), 209-246.

[4] Chemin, J.-Y.: Fluides parfaits incompressibles. Astérisque 230, 1995.

[5] Chemin, J.-Y.: Théorèmes d'unicité pour le système de Navier-Stokes tridimensionnel. J. Anal. Math. 77 (1999), 27-50. 
[6] Chemin, J.-Y. And Lerner, N.: Flot de champs de vecteurs nonlipschitziens et équations de Navier-Stokes. J. Differential Equations 121 (1995), 314-328.

[7] Danchin, R.: Local and global well-posedness results for flows of inhomogeneous viscous fluids. Adv. Differential Equations 9 (2004), 353-386.

[8] Danchin, R.: Density-dependent incompressible viscous fluids in critical spaces. Proc. Roy. Soc. Edinburgh Sect. A 133 (2003), 1311-1334.

[9] Danchin, R.: The inviscid limit for density-dependent incompressible fluids. Ann. Fac. Sci. Toulouse Math. (6) 15 (2006), no. 4, 637-688.

[10] Danchin, R.: Local theory in critical spaces for compressible viscous and heat-conductive gases. [Comm. Partial Differential Equations 26 (2001), 7-8, 1183-1233]. Comm. Partial Differential Equations 27 (2002), 11-12, $2531-2532$.

[11] Danchin, R.: Global existence in critical spaces for compressible NavierStokes equations. Invent. Math. 141 (2000), 579-614.

[12] Desjardins, B.: Global existence results for the incompressible densitydependent Navier-Stokes equations in the whole space. Differential Integral Equations 10 (1997), 587-598.

[13] Fernández-Cara, E. and Gulllén, F.: The existence of nonhomogenous, viscous, and incompressible flow in unbounded domains. Comm. Partial Diffrerential Equations 17 (1992), 1253-1265.

[14] Fujita, H. And Kato, T.: On the Navier-Stokes initial value problem. I. Arch. Rational Mech. Anal. 16 (1964), 269-315.

[15] Kazhikov, V.: Resolution of boundary value problems for nonhomogeneous viscous fluids. Dokl. Akad. Nauk. SSSR 216 (1974), 1008-1010.

[16] Landau, L. And Lifchitz, E.: Physique théorique. VI: Mécanique des fluides. Éditions Mir, Moscow, 1971.

[17] Leray, J.: Sur le mouvement d'un liquide visqueux remplissant l'espace. Acta mathematica 63 (1934), 193-248.

[18] Lions, P.-L.: Mathematical topics in fluid dynamics. Vol. 1. Incompressible models. Oxford Lecture Series in Mathematics and its Applications 3. The Clarendon Press, Oxford University Press, New York, 1996.

[19] Meyer, Y.: Ondelettes et opérateurs. III. Opérateurs multilinéaires. Actualités Mathématiques. Hermann, Paris, 1991.

[20] Peetre, J.: New thoughts on Besov spaces. Duke University Mathematics Series 1. Mathematics Department, Duke University, Durham, 1976.

[21] Planchon, F.: Sur une inégalité de type Poincaré. C. R. Acad. Sci. Paris Sér. I Math. 330 (2000), no. 1, 21-23. 
[22] Runst, T. And Sickel, W.: Sobolev spaces of fractional order, Nemytskij operators, and nonlinear partial differential equations. De Gruyter Series in Nonlinear Analysis and Applications 3. Walter de Gruyter, Berlin, 1996.

[23] Simon, J.: Nonhomogeneous viscous incompressible fluids: existence of velocity, density, and pressure. Siam J. Math. Anal. 21 (1990), 1093-1117.

Recibido: 15 de julio de 2005

Hammadi Abidi

IRMAR

Université de Rennes 1, Campus de Beaulieu 263, avenue du Général Leclerc

CS 74205

35042 Rennes cedex, France hamadi.abidi@univ-rennes1.fr 